\title{
Constructing Visual Narratives of Museum Experiences
}

\author{
by \\ Jesse Gerroir, B.I.T \\ A thesis submitted to \\ the Faculty of Graduate and Post Doctoral Affairs \\ in partial fulfillment of the requirements for the degree of \\ Master of Applied Science \\ in Human Computer Interaction
}

School of Information Technology

Carleton University

Ottawa, Ontario

(C) 2015 Jesse Gerroir 
The undersigned hereby recommend to

The Faculty of Graduate Studies and Research acceptance of the thesis

\title{
Constructing Visual Narratives of Museum Experiences
}

\author{
submitted by \\ Jesse Gerroir
}

In partial fulfillment of the requirements for the degree of Master of Applied Science in Human Computer Interaction

Chair, Anthony Whitehead, School of Information Technology

Thesis Supervisor, Ali Arya

Faculty of Engineering and Design

School of Information Technology

Carleton University

April 2015 


\begin{abstract}
The technological enrichment of museums serves as a prime area of research focused on the changing role of information and communication technologies and the visualization of personal data. While previous research projects have focused on using mobile and interactive technologies as electronic museum guides or tools for viewing additional information, in contrast, this project takes a different approach. It seeks to develop new methods to visualize the data collected from a user's trip through a museum enabling the user to construct a presentation that they can use to reflect, communicate, and share their experiences with others. The visualization they construct is not just a presentation of facts about their trip, but also a visual narrative of what they experienced. To this end, four styles of visual narratives (each targeting a particular purpose) were designed, developed, and tested via a prototype that allowed participants to explore a virtual museum and construct a visual narrative of their trip in any of the designed styles. It also allowed them to access narratives created by others. Our usability study was based upon a series of evaluation criteria such as effectiveness, operability, overall satisfaction, flexibility, and sociability. It demonstrates the users do in fact prefer the proposed narrative visualization and our visualization styles serve distinct purposes.
\end{abstract}




\section{Acknowledgements}

First and foremost, I would like to thank my supervisor Ali Arya. It seems like just the other day I was still in high school going to Carleton University's open house. He was the first person I talked to about the IMD program and he convinced me to go into the undergraduate program that eventually lead to my graduate studies, instead of the video game design stream of the Computer Science program. The IMD program sounded much more interesting and he made it feel really fun and welcoming. It has been quite the journey ever since where I have learned a lot, grown a lot, and really developed the skills, experience, and knowledge to achieve a sense of professionalism. He has been my instructor numerous time and I have worked with him on various research projects. I am grateful for the patience, guidance, and mentorship he has shown me.

I would also like to thank the entire School of Information Technology at Carleton University. They have helped support, shape, educate, and guide me throughout my time at Carleton and have always been there on a personal level.

Last but not least, I would like to thank my parents who have helped support and encourage me throughout all these years. 


\section{Table of Contents}

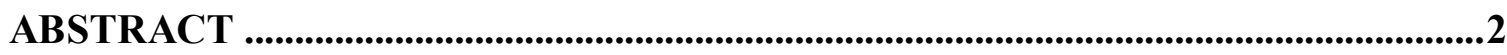

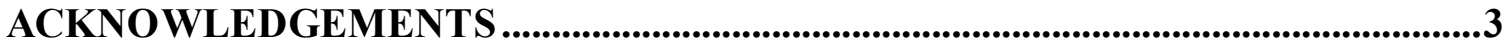

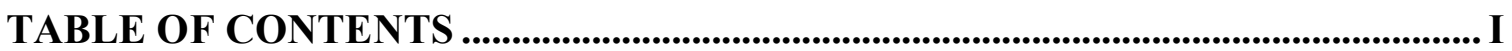

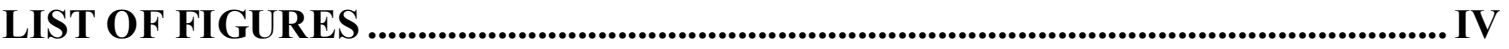

LIST OF TABLES ……................................................................................................................... V

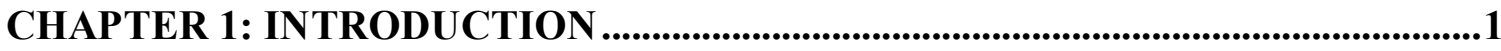

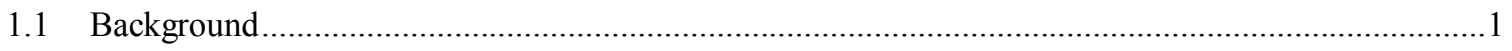

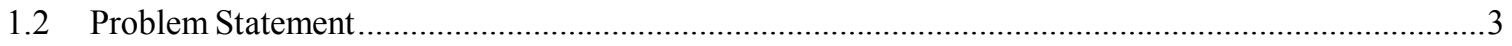

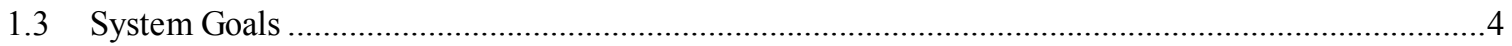

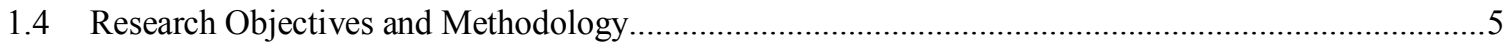

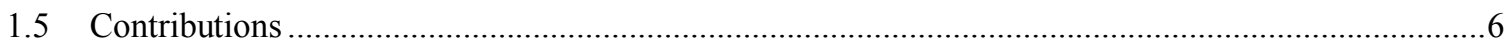

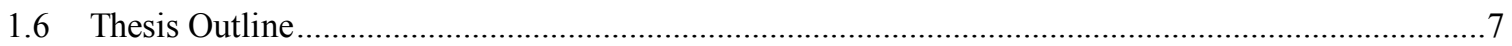

CHAPTER 2: LITERATURE REVIEW .....................................................................

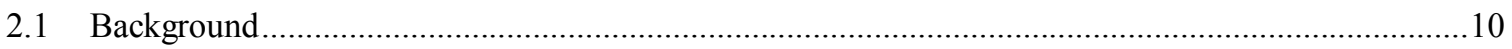

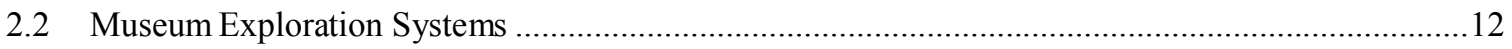

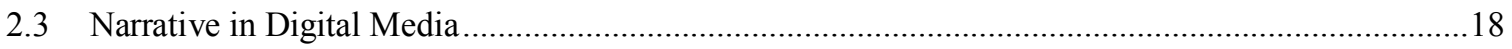

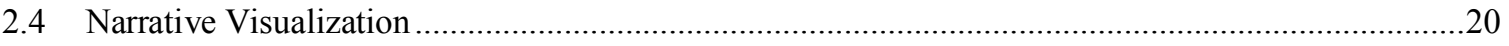

CHAPTER 3: DESIGN CONCEPTS ...................................................................................24

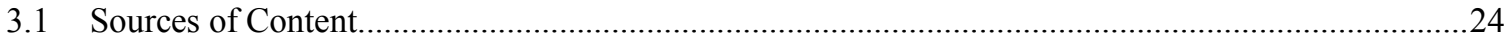

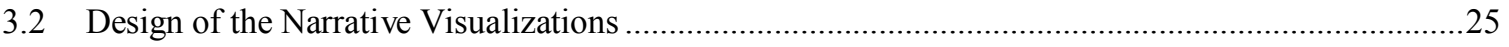

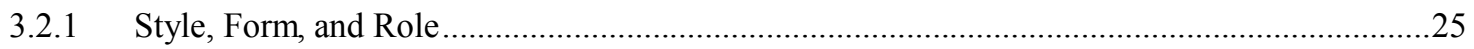

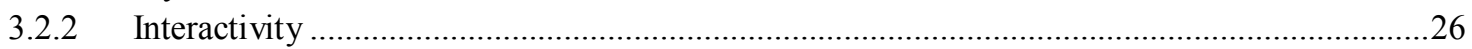

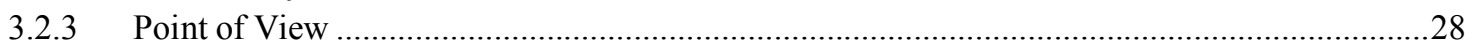




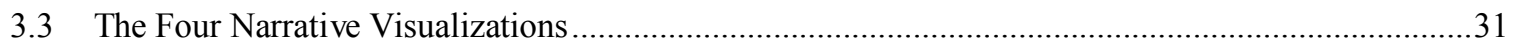

\section{CHAPTER 4: PROTOTYPE DESIGN AND IMPLEMENTATION .........................34}

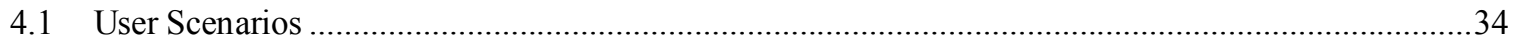

4.1.1 Scenario 1: Visiting a Museum and Constructing a Visual Narrative ............................................

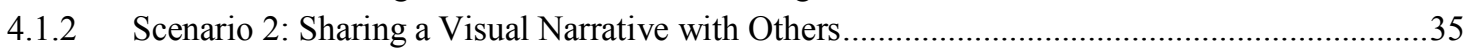

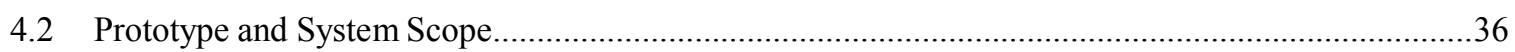

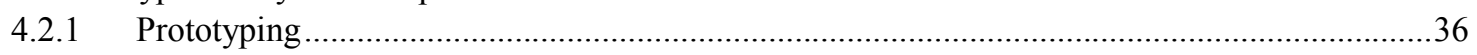

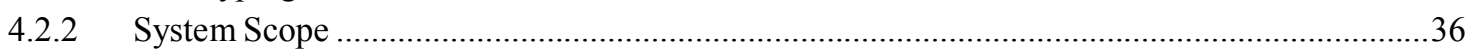

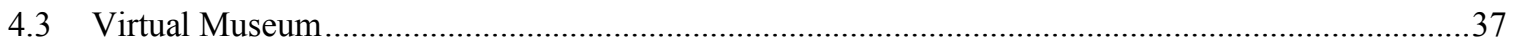

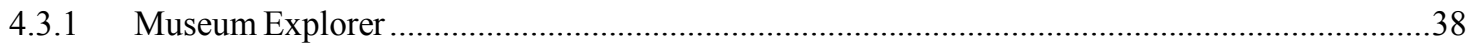

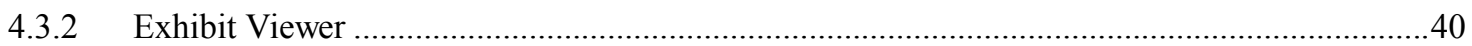

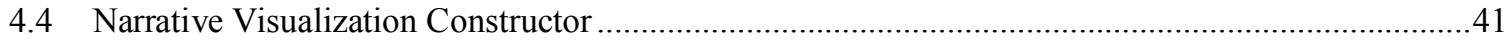

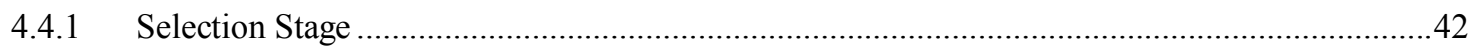

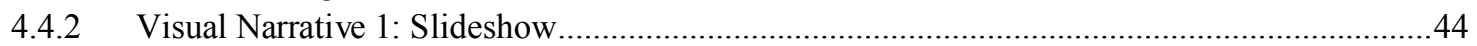

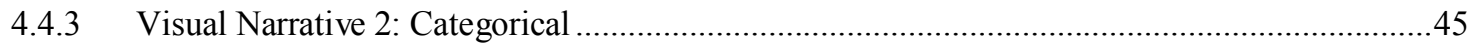

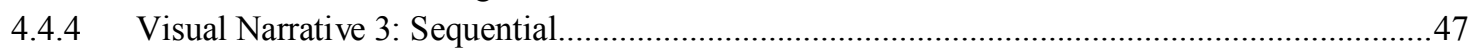

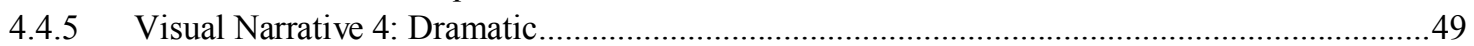

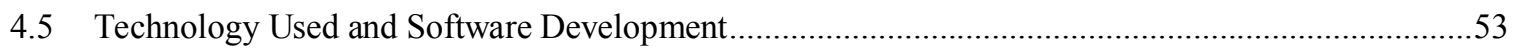

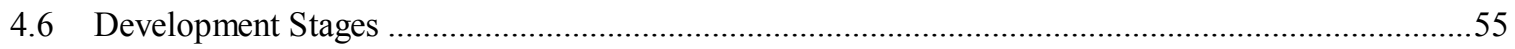

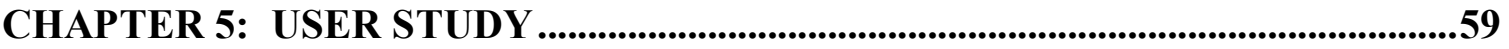

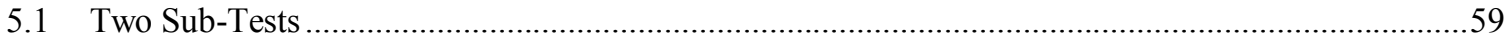

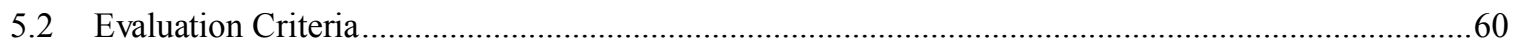

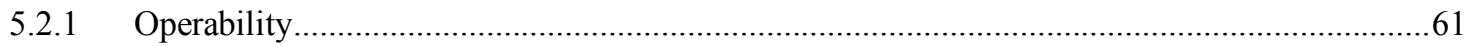

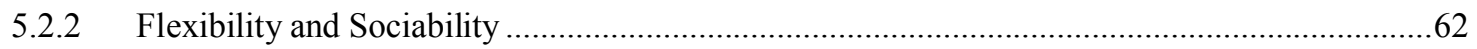

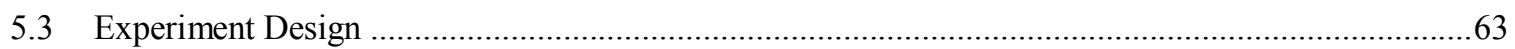

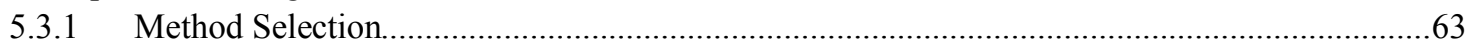

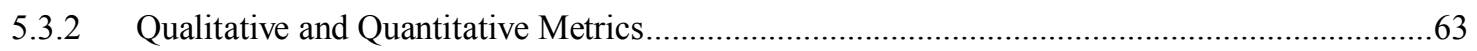

5.3.3 Independent and Dependent Variables and Within-Subject Testing .........................................64

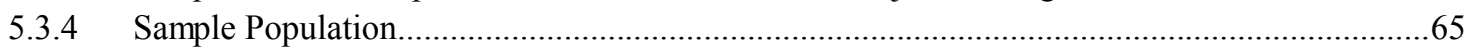

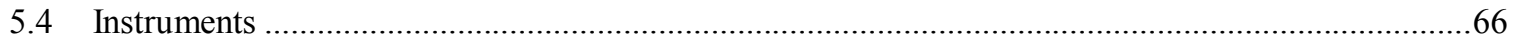

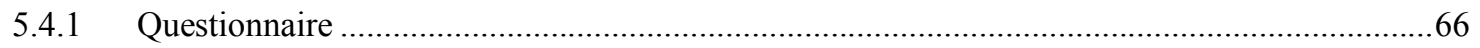

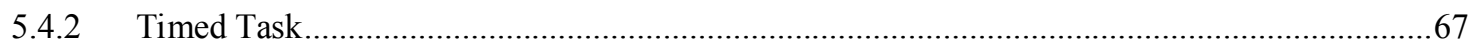

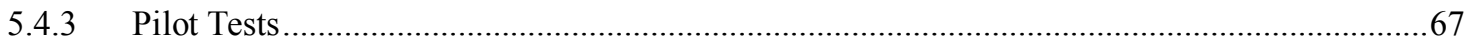

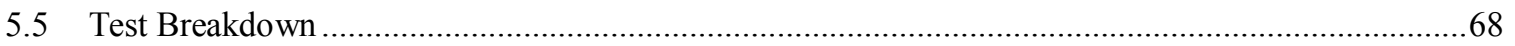




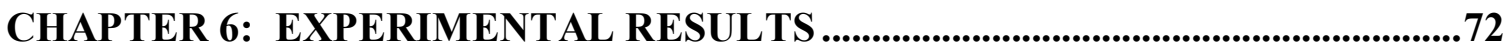

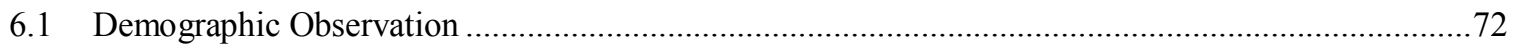

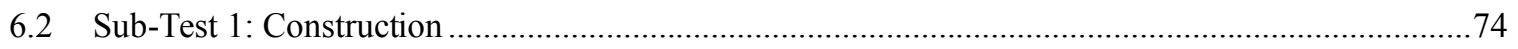

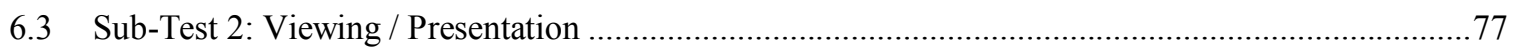

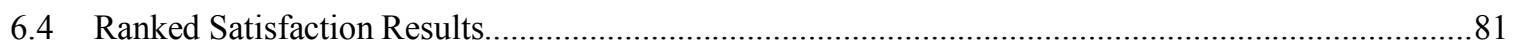

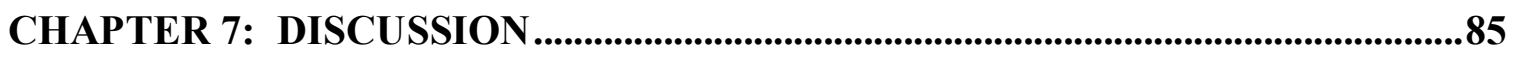

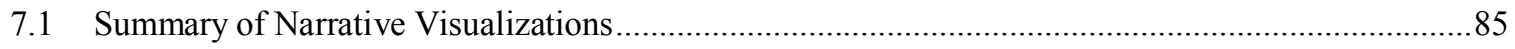

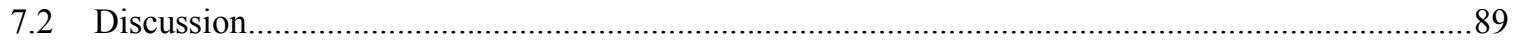

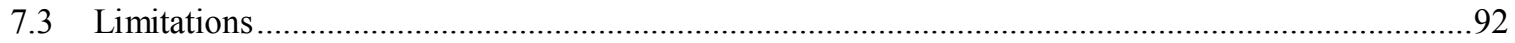

CHAPTER 8: CONCLUSION AND FUTURE WORK ..............................................94

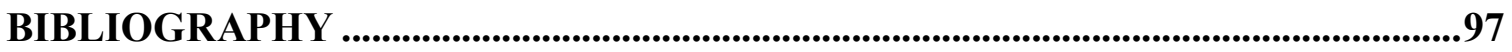

APPENDIX A: CONSENT FORM ........................................................................................101

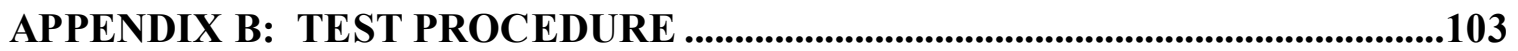

APPENDIX C: SURVEY 1 - CONSTRUCTION ......................................................106

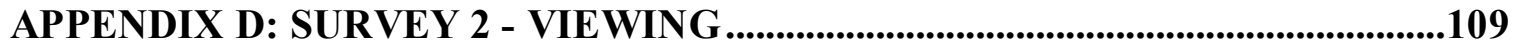

APPENDIX E: OBSERVER RECORD ...................................................................................113

APPENDIX F: HEURISTIC EVALUATION FORM ........................................114

APPENDIX G: IN-DEPTH ANALYSIS OF SATISFACTION RESULTS .............116

APPENDIX H: SOURCES OF CONTENT .................................................................122 


\section{List of Figures}

Figure 1. Information Embedded in a Narrative. [17] .................................................... 19

Figure 2. Charles Minard's map of Napoleons march on Moscow. [33] .............................22

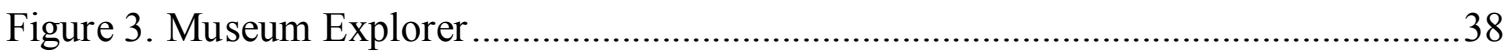

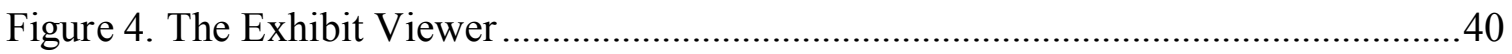

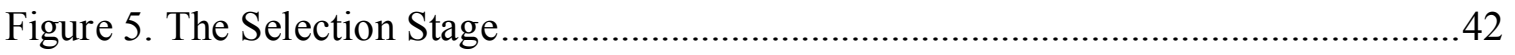

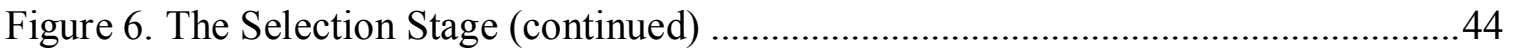

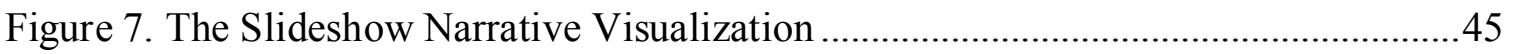

Figure 8. The Categorical Narrative Visualization (Title Page). .......................................46

Figure 9. The Categorical Narrative Visualization (Exhibit Page)...................................47

Figure 10. The Sequential Narrative Visualization (Starting Position). .............................48

Figure 11. The Sequential Narrative Visualization (Viewing an Exhibit). .......................49

Figure 12. The Dramatic Narrative Visualization (Start) ...............................................50

Figure 13. The Dramatic Narrative Visualization (Viewing an Exhibit) ...........................51

Figure 14. The Dramatic Narrative Visualization (Viewing another Exhibit) ....................52

Figure 15. Sitemap of Pages in Prototype ............................................................................53

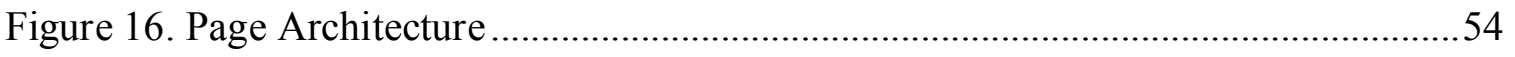

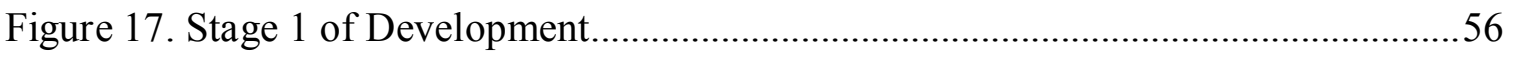

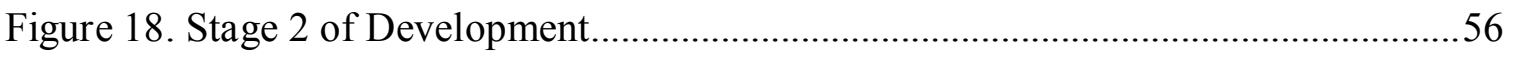

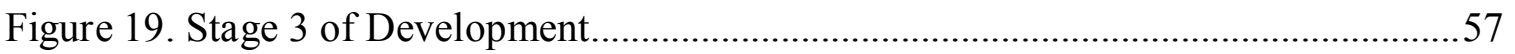

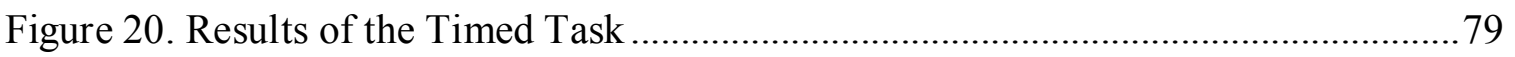

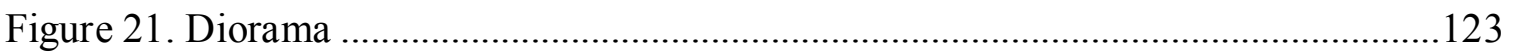

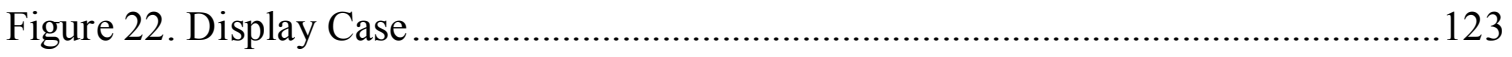

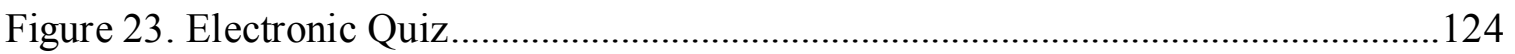

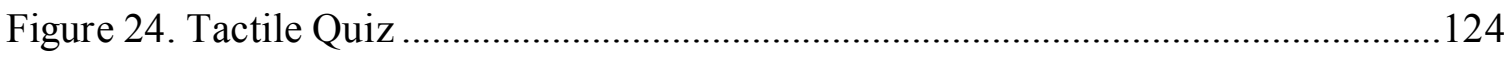

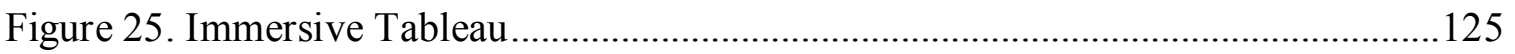

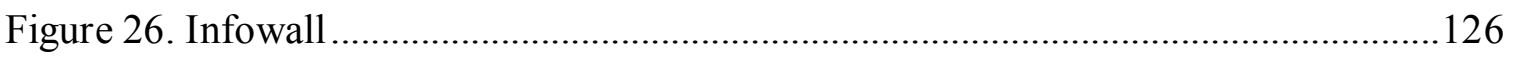

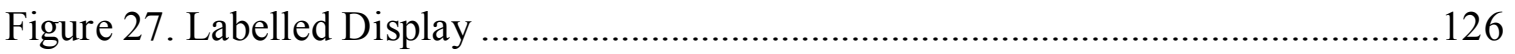

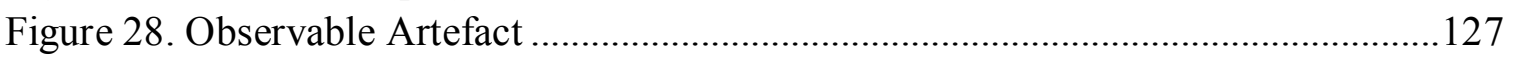

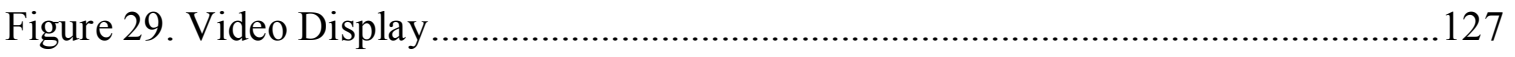

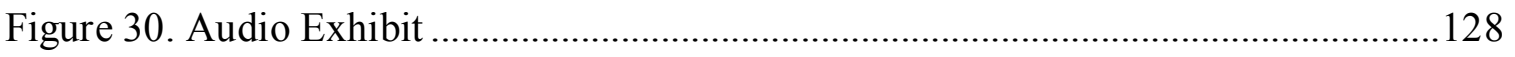

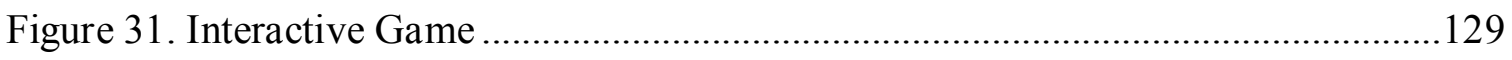




\section{List of Tables}

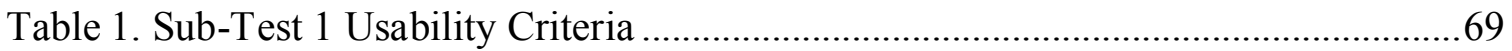

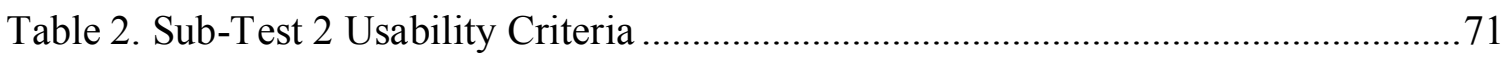

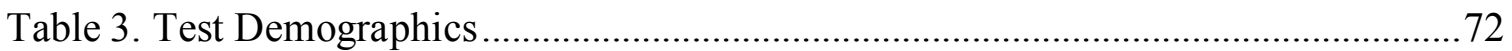

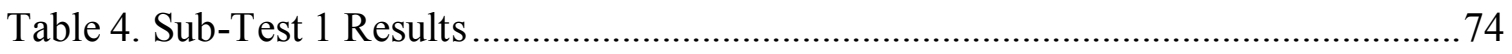

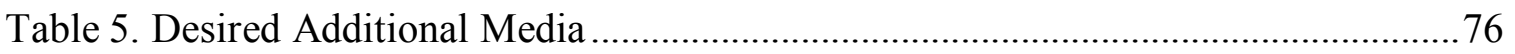

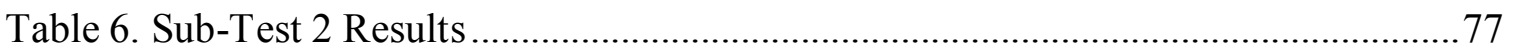

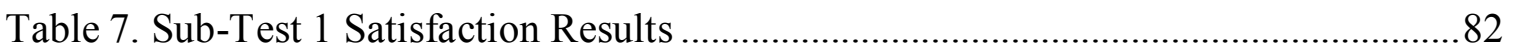

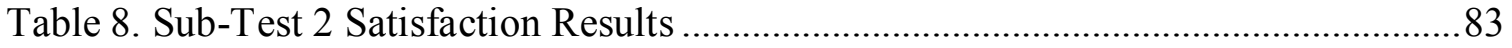




\section{Chapter 1: Introduction}

\subsection{Background}

Museums today are often complex institutions that range in scale from a heritage home run by a single individual, to a large multi-building facility that has ties to academia and the government. The collections museums house can also vary depending on the theme of the museum. They can range from art galleries, to children's museums, to science centers, to history museums, and much more. Regardless, as defined by the International Council of Museums:

"A museum is a non-profit, permanent institution in the service of society and its development, open to the public, which acquires, conserves, researches, communicates and exhibits the tangible and intangible heritage of humanity and its environment for the purposes of education, study and enjoyment." [1]

To this end museums serve to offer their visitors an educational experience, through exposure to and interaction with the artefacts they house [2]. Since the 20th century, museum research has expanded beyond collections to include museum practices and the museum's visitors themselves. Alexander \& Alexander [2], explain that:

"...museums have moved beyond collections and collecting so dominant in the 19 th and early 20th centuries, to become institutions rooted in interpretation in its broadest sense, actively seeking to provoke thought and the exchange of ideas between the museum and its visitors." 
"Exhibition, education, or interpretation-the conveyance of culture-and a commitment to community or social welfare has grown to be important aims for the museum in the last century."

The modern museum offers many ways for the user to interact with the museum ranging from an online search of the collections it hosts before the visit, to touch screens and interactive displays within the museum, to multimodal and more hands-on interactive systems that allow users (especially children) to learn in a playful manner [3]. As the relationship between users and communication technology has changed from simply receiving broadcast messages(such as in the era of TV) to interactive engagement (such as with the advent of the Internet), so too has the museum.

Mobile telephony has further developed this relationship by creating point-to-point networked interactions. Messages, content, and interaction are calibrated for the specific individual and transform how the individual orients in space and time $[4,5]$. The personalization of information and a sense of the individual rather than location tend to be the defining effects of mobile communication:

"...the widespread adoption and use of mobile communication technologies mark a new phase in the network society, chiefly characterized by heightened personalization in the relationship between technologies and their users. With these technologies we call to individuals and not to locations." [6]

Museums have always sought after interactive technologies to improve the visitors experience and heighten the interaction of the collection. The technological enrichment of museums serves as a prime area for research. How modern mobile interactive 
technologies can be used to gather and present personalized information offers new opportunities for more effective and attractive interactions and communications between museums and their visitors, and also the visitors among themselves.

\subsection{Problem Statement}

The creation of a system to help facilitate the interaction and exploration of a structured space is not a new topic. Several such systems are reviewed in detail in Chapter 2: Literature Review. However, most previous research focuses on technology to guide the user or present them with additional information about the exhibits and often serve as electronic tour guides. They don't use or explore advantages in the advanced information and communication technologies to move from simple "mobility" to a "personalized" framework, i.e. call to individuals instead of locations [6].

There are other features missing in previous proposed systems. Museums often overwhelm the visitor with information. Everyone tends to want to view and learn something and while different objects in a museum can resonate greatly with individuals in the moment, users are often mentally and physically exhausted after their visit. It becomes hard for them to easily remember or articulate specifics of what they learned. It is only after some time later that some other object, event, or story will bring back the memory of the experiences they had within the museum [7].

This thesis seeks to address some of the above issues through the design, development, and evaluation of four different visual narratives. Explained fully in the literature review, visual narratives are presentations using text, multimedia content and often interactive 
elements, to convey a narrative. Narrative visualization doesn't just seek to use images to illustrate a story. It seeks to fundamentally convey information through both textual and visual narrative. It tries to present the data in a holistic fashion [25].

This research project seeks to study how to best allow visitors to construct one of four visual narratives that enable them to reflect upon and communicate what they experienced in a museum and share and present their experiences in a museum with another.

\subsection{System Goals}

The system goals can be concisely defined as the following in regards to user context and functionality:

Construction: The system should give the users the ability to take the data they collected while in a museum and construct one of four visual narratives to represent their experiences within the museum.

Reflection: The designed narrative visualizations should allow users to remember and reflect upon what they experienced and learned within the museum, through them the information is presented in a holistic fashion rather than as individual elements or purely quantified data.

Communication: The designed narrative visualizations should be pleasant to look at and clearly communicate what happened to the user in the museum. They should grant a clear understanding of the person's chosen narrative within the museum. 
Sharing: The four narrative visualizations should go beyond just communicating their contents clearly. They should be in a format that is easy to share with others, evokes conversation, can be used to present the persons experiences, and overall foster a sense of social interaction. The user should feel encouraged to share their experiences and have others share with them.

\subsection{Research Objectives and Methodology}

The main research methodology of this study was to develop a prototype to use for usability testing. Our approach included determining a set of goals through analysis of users' needs, designing a series of visualization styles and a system to offer them, developing a prototype, usability testing, and finally evaluation of the system through well-defined criteria. This approach was based upon previous research fully detailed in Chapter 2: Literature Review, as well as HCI methodology outlined in Usability Engineering by J. Nielsen [40] and Human computer Interaction by Dix et al. [41].

For this study two specific research questions were defined to be answered through analysis of the results and evaluation. The research questions were chosen to explore how successful the narrative visualizations would be in comparison to traditional methods and to each other. They are the following:

Question 1: Would each of the four narrative visualizations succeed at conveying its content in a satisfactory manner to the users?

Question 2: Would the users prefer the 'new' narrative visualizations (categorical, dramatic and sequential) to the slideshow and traditional method? 
Concisely stated the research methodology is the following:

1. Identify visualization styles that match various exhibit types and purposes, through literature review and museum visits

2. Design and develop a prototype that embodies the system goals and which can perform tasks related to the user scenarios.

3. Perform usability testing on this prototype based on the usability criteria including effectiveness, operability, flexibility, sociability and satisfaction

4. Evaluate the results in order to answer the research questions

\subsection{Contributions}

The main contributions of this study are:

1. Identification and design of four major narrative visualization styles. Based on the literature review and analysis of users' needs four narrative visualization styles have been designed which include: Categorical, Slideshow, Dramatic, and Sequential.

2. Design of a museum system that supports the museum's main goals: The main goals of the constructed prototype were to facilitate the construction process of the visual narratives and to embody the system goals of construction, reflection, communication, and sharing. In this manner the four narrative visualizations are meant to align more with the goals of a museum and focus less on tour guidance tasks which many other systems focus on. 
3. Usability testing of four different narrative visualization schemes: Each of the styles incorporates a different narrative structure and visualization genre. They were designed based upon research into narrative visualization and narrative in digital media. In this way this study helps contribute to narrative visualization and its results serve to help further narrative visualization methods and ideas.

\subsection{Thesis Outline}

The thesis is divided into 8 chapters and several appendices. A brief description is as follows:

\section{Chapter 1: Introduction}

This chapter provides some background information about the research topic, the problem statement that this survey seeks to address, and the goals of the system that this research project seeks to create through the development of a prototype.

\section{Chapter 2: Literature Review}

The literature review begins by detailing the previous project, the MEseum that is used as a starting point, and then reviews the three main topics that were researched: museum exploration systems, narrative in digital media, and narrative visualization.

\section{Chapter 3: Design Concepts}

This chapter covers ideas and research that went into the design of the narrative visualizations. It includes a discussion of theories, ideas, and methods from the literature 
review that influenced the design of the prototype and an explanation of the conceptual narrative visualization styles.

\section{Chapter 4: Prototype Design and Implementation}

This chapter explains the prototype in detail. It begins by explaining the two user scenarios that formed the backbone of the functionality then delves into the functionality of the prototype detailing the virtual museum and the narrative visualizations. It explains how the narrative visualization styles introduced in the previous chapter were implemented.

\section{Chapter 5: Research Approach}

This chapter covers the research approach, it begins by explaining how the usability test is comprised of two subtests and then explains each test in turn detailing the usability criteria, tasks, and metrics used to evaluate the participants.

\section{Chapter 6: Experimental Results}

The test was carried out on 20 participants. The results are presented here by test grouped by test and evaluation criteria.

\section{Chapter 7: Discussion}

In this chapter the results of the experiment are analyzed along with a discussion of research questions and limitations of the survey. 


\section{Chapter 8: Conclusions and Future Work}

This chapter summarizes the results in the context of the original research questions and suggests directions for future research on the subject. 


\section{Chapter 2: Literature Review}

\subsection{Background}

The MEseum project was started by Efetobore Mike-Ifeta, a graduate student in Human Computer Interaction at Carleton University. The general purpose of the MEseum project was to design and develop an interactive system that would include new ideas and tools catering to the new generation of museum-goers. Central to its development was the concept of using a narrative as a key element in the system. To this end, the proposed system, MEseum, offered various tools to the museum visitor that allowed for the authoring, curating and sharing of museum experiences [8].It had three main goals regarding its implementation:

Planning: "Planning a visit based on user interests, exhibits, and input from other visitors."

Guidance: "Following the planned visit through some method of tracking while artefacts related to various steps can be collected and added to the narrative."

Reflection and Connectivity:"Connecting to other members before, during and after the visit." [8]

MEseum included planning and guidance modules and the concept of timeline as a way to collect and share information related to a visit as a personalized narrative. For usability testing, a proof of concept prototype was developed. Test participants were asked to complete a user task comprised of: planning a simple tour path, building a timeline of their museum experience as they explored a museum, and viewing some of the mocked- 
up social media functions. They then were asked to complete a survey, giving feedback on the session and the concept itself.

The usability testing had a number of findings. Overall, there was a positive response to the purpose and functionality of the MEseum system [8]. Participants embraced the idea of having an online community of people with similar interests that could act as support for the process of constructing digital narratives [8].

However, it being a proof of concept prototype, there were a number of issues observed by the participants regarding the design of the system. Many participants believed that the design and usability could be improved [8]. As a result, while there was positive support and feedback for the concept of the system, the MEseum study made two main recommendations for further development:

1. A more qualitative or mixed method of study would probably be more suitable for the study [8].

2. The isolation of different components of the system for redesign and usability testing $[8]$.

To this end at the start of this research project the MEseum system was broken down into a number of components and analyzed. The timeline component was identified as a candidate for further research; it could become a much more interactive experience that captured and contained a lot more data of the users visit. It was selected as the component that was to be developed in this research study because of its key role in improving the experience through storytelling and narrative visualization. 
The timeline was analyzed further. The described functionalities of the timeline feature in the MEseum system are the following:

- Collect content related to the user's experience.

- Present or visualize this content.

- Allow the user to reorder, reflect, revisit, and share this presentation with others.

However, the timeline presentation was very limited in MEseum and only included a simple slideshow of captioned photos. There was room to develop it further including a better study of presentation styles. Thus the timeline served as the starting point and main focus for this research study.

\subsection{Museum Exploration Systems}

As mentioned in the problem statement, most other systems tend to focus on technology to guide the user and provide additional information. In this sense they overlook the psychology of the user in the museum and the sociology of the user with other people both during and after their visit. There are some exceptions, most notably the MEseum Project and Urban Tapestries. Both of which try to foster social interaction and the sharing of social data. However both lack components that deal with the psychology of the user in the museum that goes beyond simple ease of use and usability.

This project doesn't have as grand sociological goals as those two systems and is more interested in user psychology instead. The system should go beyond trying to understand user psychology in terms of usability and what tasks they are most likely to perform in a 
museum, to trying to support the cognitive goals of the museum such as learning and reflection.

A breakdown of each system surveyed is explained as follows with a discussion of their drawbacks and benefits.

\section{The MoMo}

The MoMo [9] enriched the museum visiting and learning experience through tailoring digital content to the user. The device it used was Personal Digital Assistant (PDA). PDA's allowed the user to select and modify tours throughout an art gallery. The main problem it sought to address was the amount of information a user has access to about an exhibit. The system designers wanted the user to be able to query additional information but not in a way that would compete with attention for the tangible artefact.

They identified two main hierarchies of information navigation, horizontal and vertical. In horizontal navigation, the user views other artworks that are associated with the one they are viewing, such as what other works in the art gallery the artist had created. In vertical navigation the user seeks to learn additional information about the painting they are currently viewing, such as when it was made, who made it, etc. While one hierarchy is more associative, the other is more detailed. In this manner the system would offer this information and allow the user to navigate as they willed.

A secondary feature of the system was that it would keep track of the users' selections and navigations and would use algorithms to estimate what the user was most interested in. The system would then prominently display icons related to that information over others. This project's identification of the two main hierarchies of information, horizontal 
and vertical, was very interesting and its algorithms helped the user from being overwhelmed. However, in more traditional museums, unlike art galleries that tend to just have paintings and very little info, the system might not be as useful. Most of the information would already be displayed in the museum as part of the exhibit and structured to guide the user through it.

\section{The CHIP Project}

The CHIP interactive guide [10] was a tour guide project for museums. Like the MoMo, it was implemented in an art gallery. The main problem it sought to address was the creation of individual tours. The user would begin their experience before their trip by using an online art recommender offered by the museum. The online art recommender would recommend art in the art gallery that the user was interested in by allowing the user to select different paintings that they wanted to see and then suggesting further paintings. Once the user was satisfied, it would create a tour that the user could follow in the museum on a mobile device.

All the paintings on their tour were overlaid on a map of the museum and the user could dynamically alter and adjust their tour as they progressed. Overall the system excelled at starting the museum experience before the person visited the museum and continuing it while in the museum. While many museums do have websites with information about their contents by integrating the planning functionality with a mobile application, it let the user access their plan once in the museum. It also let the user create an individual tour with the art recommender with the ability to add or drop paintings they wanted to see on 
the fly. However, there was no emphasis on narrative visualization, or general data collection and sharing in CHIP.

\section{The Gentle Guide}

The Gentle Guide [11] was a bit different from the other systems in that it wasn't meant primarily for museums but instead was meant for general indoor navigation. It was a wearable device that would guide the user using haptic vibration. The user would wear wristbands on the left and right wrist. Each wristband would vibrate depending on which direction the user needed to turn, and vibrate on both wrists to signal stop. Most users found it to be an intuitive system. What is most interesting regarding this study is its approach to 'heads down' navigation. The tangible artefacts in museums tend to be the main reason people visit them. Most systems rely on visual feedback to guide the user which causes them to have to look down while they navigate or view extra information on the device. This system eliminated this 'head down' navigation and the competition of attention between the system and the museum. Again, planning and construction of a narrative was beyond the scope of this system.

\section{The CAERUS}

The CAERUS [12] is a context aware educational resource system. It differed from the other systems surveyed in that it was used for outdoor tourist sites. It allowed users to explore a large outdoor area like a park either with a goal or free form. The user would be presented with a map of the area that is divided into regions with each region having a different theme. 
Whenever they entered a new region the user would be presented with audio content and could view additional content relating to the region. Each theme would group one or more regions based upon a certain perspective such as 'history' or 'environment'. The user could choose to guide themselves or follow a specific path.

The main feature of the system was that through the audio commentary of certain regions it attempted to be both a touristic and an educational system. It allowed the user to freely roam and would adapt to where they were informing them as they went along. While great for large outdoor environments with little signage or physical displays, the system might not be as suited to indoor spaces. However, its categorization of the information into regions tied to geography, and organization of regions by theme, was a very interesting way of structuring the information and allowing a user to explore it in a freeform manner.

\section{Urban Tapestries}

Urban Tapestries [13] is a public authoring application that allowed users to leave messages, notes, observations, and other comments on a map to be seen and read by other users. While not meant for museum exploration or guidance specifically, it attempts to create a very visual presentation of its users' data through displaying it all on a map using lines and dots. Thus it would create a virtual tapestry where each thread is related to the data of a specific user. It is an interesting system for unlike the other systems discussed, it has more social goals; such as the building a community through the building up of local knowledge about an area and the creation of a public dialogue via facts or comments about an area. 
This community or social aspect of a space is often overlooked in most museum systems. They seek to make the museum interactive for the visitor but often don't incorporate interaction with other users, neither incorporate the experiences of other users. Also, from a data visualization perspective, the presentation of the information with lines and nodes was unique and allowed people to intuitively see which areas were the most popular through the amount of annotations and threads criss-crossing them.

While this research project has social goals they are lesser in scope than the ones of Urban Tapestries were. The sociological goals of this project are more limited to how a user can best present their visit, rather than the building of a local community or local community knowledge. Additionally unlike urban tapestries this project seeks to study the psychology of the user in regards to how the narrative visualizations can help facilitate learning and reflection.

\section{MEseum Project}

The MEseum project [8] is an interactive museum guidance application that allows users to plan a route before they visit a museum, follow their route via an interactive map, and check in at exhibits as well as chat with other people in the museum. When checking in at exhibits the user could upload photos and comments about their experience and the system would build a timeline of places they checked in at. This timeline would serve as a record of their experiences. It sought to create a system that covered the entire experience, from planning a tour before the trip, to being guided within the museum, to having a timeline to represent the experience after. 
Overall it sought to present the museum visit in a holistic manner. This would enhance remembrance and sharing of the trip and therefore indirectly help further the goals of museums of being places of learning and interaction.

\subsection{Narrative in Digital Media}

The benefits of narrative are multiple. Narrative has great potential in enhancing learning as seen in the works of Burner $[14,15,16]$, and as explained by Gershon and Page; narrative and storytelling allow us to present information more effectively [17] and to perceive information, such as emotions, judgements of character, themes, values, that are very hard to convey otherwise.

Figure 1 shows how a very short story containing a relatively large amount of information. We can see how that information becomes tedious to absorb when presented in more straightforward bulleted list format: 


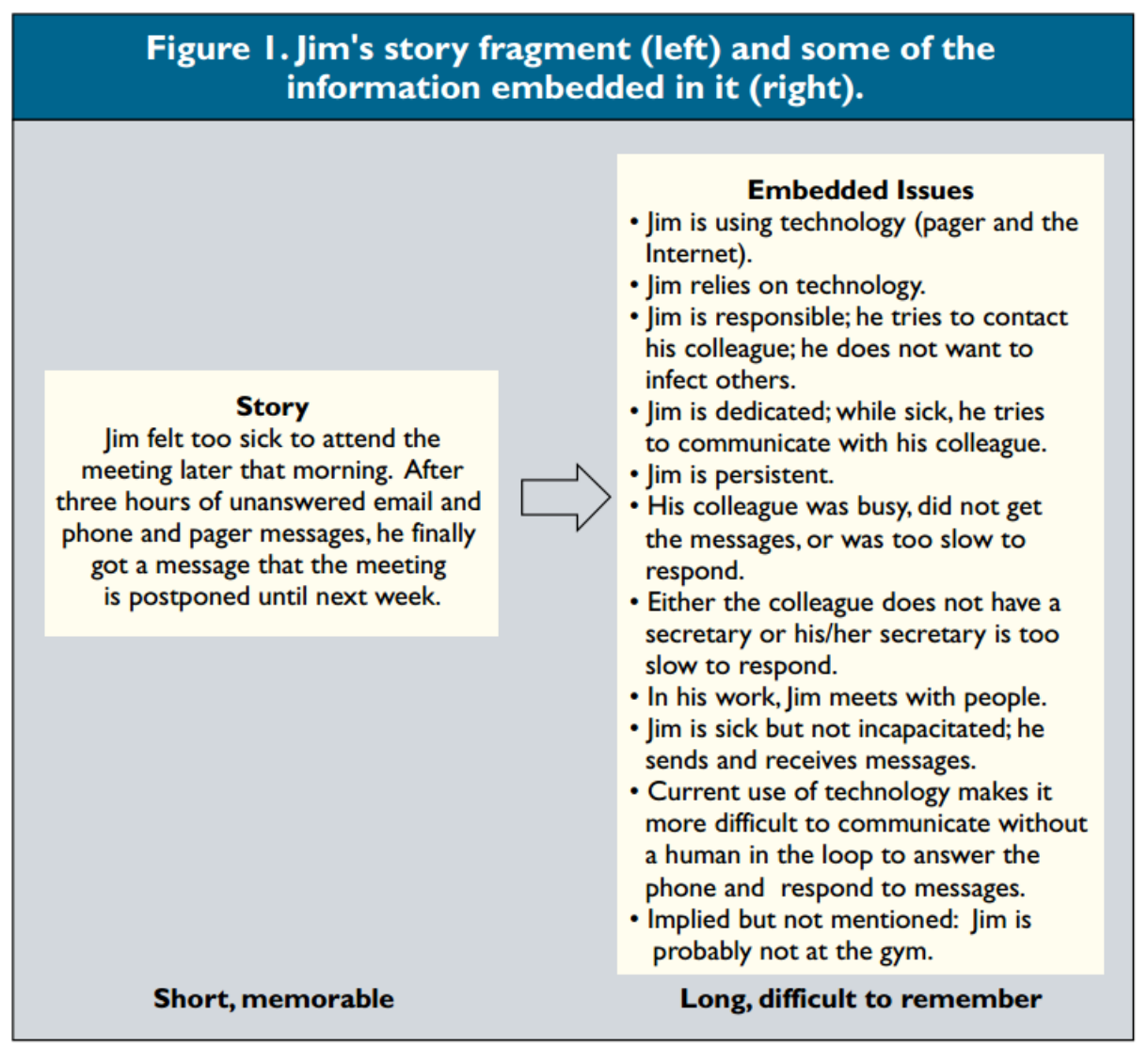

Figure 1. Information Embedded in a Narrative. [17]

To this end, using a narrative to present a visit to a museum would allow the structuring of information in a beneficial manner. Furthermore museums themselves have long used stories and narratives for conveying information related to their exhibits and artefacts, and so the use of narrative in this domain doesn't come as a surprise or something that the user has no conception of.

In a traditional narrative you generally need at least two events, one occurring after another and the events must be entities that contain action. A dog with fleas is not an event, a dog getting bit by fleas is an event with action [18]. The actions and order of events can vary in each story but form the plot of the narrative. In this manner, a narrative is not just a presentation of facts but a sequence of occurrences. 
In Beyond Myth and Metaphor: The Case of Narrative in Digital Media [19], MarieLaure Ryan discusses this. While the actions of the protagonist in traditional narrative are dictated by the author, in interactive digital media the user of the system is often the protagonist. The actions of the user tend to create much of the plot in the narrative, and the goals of the user are often the goals of the protagonist of the story. To this end, narrative in digital media, especially interactive media, is often quite different from traditional models $[20,21,22,23,24]$.In interactive digital media the narrative often evolves over time, sometimes unpredictably, rather than being a set predetermined sequence of conflicts.

Marie-Laure Ryan discusses two key elements of digital media that are important factors. The first is the increased level of interaction, which makes traditional methods of storytelling and story creation very difficult. The second is the point of view of the user in regards to interactivity. Differing points of view will shape how the user interacts with the media and the interface to do so. Different variations of these two elements can change how a narrative is composed in digital media in a drastic fashion and are discussed in detail in Chapter 3: Design Concepts.

\subsection{Narrative Visualization}

Thus far, only traditional and digital narrative has been discussed. However, narrative visualization doesn't just seek to use images to illustrate a story. It seeks to fundamentally convey information through both textual and visual narrative. It tries to present the data in a holistic fashion [25]. 
As detailed by Robert Kosara and Jock Mackinlay [25], storytelling is seen as the next logical step for data visualization. They expound upon how an assumption often made in Information Visualization literature is that the tools used for exploration or analysis are suitable for presentation. The central goal of data analysis tends to be the discovery of trends and insights. However, once discovered, these visualization tools tend to be less useful in providing a visual presentation of the findings [25].

They draw upon online journalism to provide an example of this. Online journalism is increasingly incorporating video, pictures, and other highly visual data into stories [26]. There are two general phases in this process. First, data is collected through research and interviews and analyzed to discover key events, facts, and people involved. Then this data is tied together into a story. Much of the material gathered in the research phase; hours of video footage, pages of notes, doesn't make it into the final presentation and serves as raw background information. In this manner the visualization of narratives requires its own set of tools and methods that are different from those used to gather and analyze data in more traditional visualization. For, unlike traditional visualization, the goal of narrative visualization is often not to discover but to guide.

Graham Dove and Sara Jones [27] examine the guided nature of narrative visualization. Information visualization tends to be interested in the discovery of insights. Tools are provided to visualize data and to allow a person to analyze and process the data in order to discover relationships between elements and discover insights. The data visualization field has long researched different techniques on how to best do so given different databases of information [28, 29, 30, 31, 32]. With narrative visualization, the users aim 
at sharing a narrative with others in addition to reflecting on it themselves in order to discover new insights to the experience.

While narrative visualization might seem like a new idea some of the earliest data visualizations were aimed at presentation rather than exploration or analysis. One of the first examples of data visualization, Charles Minard's map of Napoleons march on Moscow [33], was used primarily as a means of showing the effects of the harsh Russian winter on Napoleons soldiers and their dwindling numbers as the march went on.

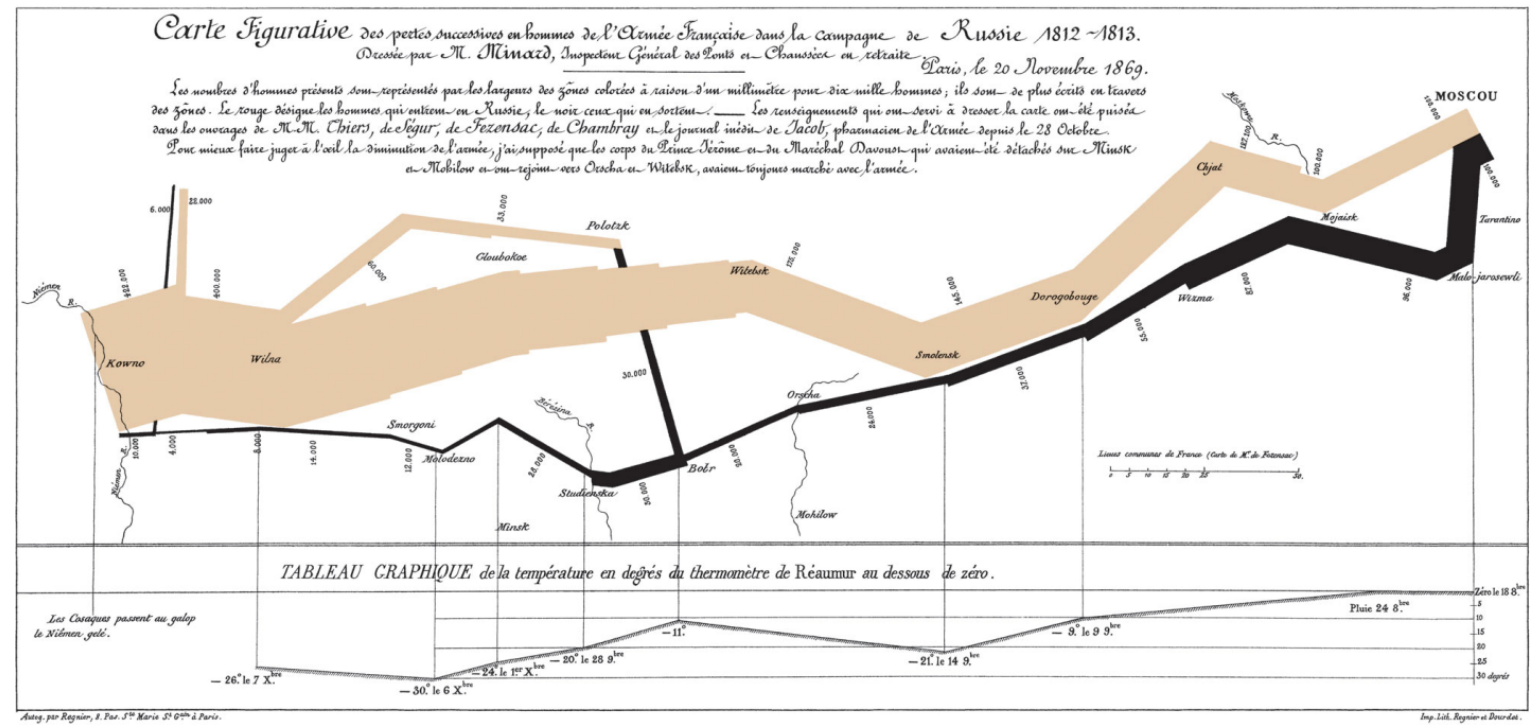

Figure 2. Charles Minard's map of Napoleons march on Moscow. [33]

In more modern times, narrative visualization techniques are often used to shape rhetoric, a topic explored by Jessica Hullman and Nick Diakopoulos [34]. They examined how, in a visual manner, data can be framed and contextualized by the creator (be it designer designing one or a user using a tool to create one) of the narrative visualization in a way that supports one interpretation at the expense of another. 
To identify and classify narrative visualization techniques, Edward Segel and Jeffrey Heel [35] studied different narrative visualizations in print and online media. They found several different patterns that involved various aspects of presentation such as layout, story structures, and structures used in conveying the story in a visual manner, sometimes with interactive components. Most interestingly, they found seven different 'genres' that were commonly used in the media to present narratives visually. These genres function as general visual metaphors for interaction and presentation and are discussed in full in Chapter 3: Design Concepts. 


\section{Chapter 3: Design Concepts}

\subsection{Sources of Content}

Some preliminary discussions and background work were had at the start of the project about what sources the narrative visualizations could use for their content. They are mentioned here only briefly, as they did not have a major influence in the final design. For reference, these sources are presented in full in Appendix I.

There are four possible sources that the visual narratives could draw from: the museum itself, the user, other users, and outside sources. These different sources could provide different types of information to be visualized.

However, the museum itself was seen as the primary source where most of the content in the visualizations would come. To this end several museums were visited with the purpose of classifying the type of exhibits that were common in them and the type of information the user could collect from them.

The museums visited were the Canadian War Museum [36], The Canadian Museum of Nature [37], and The Canadian Museum of History [38], all located in Ottawa, Canada or the nearby city of Gatineau, Quebec.

There were 11 different exhibit types that were classified. They are: Diorama, Display Case, Electronic Quiz, Tactile Quiz, Immersive Tableau, Infowall, Labelled Display, Observable Artefact, Video Display, Audio Exhibit, and the Interactive Game. All of them are described in detail in Appendix I: Sources of Content. 


\subsection{Design of the Narrative Visualizations}

\subsubsection{Style, Form, and Role}

In researching the topic of narrative in new media, different museum systems, and information visualization, it became clear that while there was overlap between each topic, each would be helpful to inform and develop a different aspect of our initial design.

The differences between the topics and how they would be applied can be outlined in a division made between the style, form, and role that the narrative visualizations would take:

The style is the look and feel the narrative visualizations would take and the techniques that would be used to present the content. The narrative visualization research outlined in the literature review helped inform how to present narratives visually. It was used to develop a unique thematic look for each narrative visualization.

The form of the narrative visualizations is the structuring of its content, i.e. the way and order in which the facts of the experience are presented. In the literature review, the narrative in digital media research, helped inform how to develop different ways of structuring the users content.

The role of the narrative visualization is the overall purpose the visual narratives will play within the system. Analysis of the timeline feature from the previous survey, and research into different museum guidance and exploration systems, helped inform and formulate ideas as to what the role would be. In this research project, the narrative 
visualizations serve as presentation tools that meet the system goals outlined in the Introduction. The basic role of the narrative visualizations doesn't change much from one style to another, unlike the form and style.

For an example of this distinction made between style, form, and role, take the slide show narrative visualization detailed further in this document. The style it takes would be that of a slideshow; its data is large pictures presented one after each other. It displays titles or comments associated with the pictures but besides this contains no other text. The form narrative takes would be the ordering of pictures chronologically and having long pauses on all of them so that the user can explain to others what is going on in the picture before it transitions to the next one. The role would be to serve as a presentation tool that meets the goals outlined in the Introduction. In this manner the style, form, and role of the narrative visualizations are three interrelated but distinct aspects of the narrative visualization.

\subsubsection{Interactivity}

While interactivity is not limited to digital media most traditional narratives are not very interactive; the user simply reads the story or watches the movie. The ability of the user to interact with the narrative visualizations was an important consideration in the design and the form the narrative would take. In Beyond Myth and Metaphor: The Case of Narrative in Digital Media [19], Marie-Laure Ryan identifies two different main modes of interactivity in digital narrative: 
"In the exploratory mode, the user is free to move around the database, but this activity does not make history nor does it alter the plot; the user has no impact on the destiny of the virtual world." [19]

"In the ontological mode, by contrast, the decisions of the user send the history of the virtual world on different forking paths." [19]

In these interactive modes, the users are able to make choices based on information they are presented with. However these two different modes can widely shape the narrative in different ways.

With the Exploratory mode the user is able to move freely about the information but does not alter the plot. A good example of this would be an interactive system where the user could hover and travel around a historic battle and be able to choose what they want to see as it unfolds. While the user has no input on the actual course of the battle, they are able to choose what they want to learn about and what conflicts and characters they want to follow. In this manner they are able to interact with the system and create a narrative based upon their interactions. Different users would come away with completely different narratives about exactly what happened during the battle even though they had no input on the unfolding events.

With the Ontological mode, in contrast, the user is able to make choices and affect the outcome of the narrative. An example of this would be being able to follow a group of characters over the course of a battle and make decisions about what actions the characters, which take would directly affect the outcome of the battle and the general narrative. 


\subsubsection{Point of View}

Besides interactivity, point of view is another element of digital media that heavily affects the structuring and presentation of a narrative and was a consideration when designing the narrative visualizations.

While point of view does exist in traditional media and is one of its elements, it takes on a different meaning in interactive digital media [19] because of the increased interactivity. In digital media where the user has the ability to interact with the system, the user can become a character or part of the story themselves. This would be impossible in traditional media.

Marie-Laure Ryan [19] identifies two different points-of-view of the user:

"In the internal mode, the user projects himself as a member of the fictional world, either by identifying with an avatar or by apprehending the virtual world from a first person perspective."

"In the external mode the reader situates himself outside the virtual world. $\mathrm{He}$ either plays the role of a god who controls the fictional world from above, or he conceptualizes his activity as navigating a database.”

Both these modes are interactive, but the point of view will drastically alter the access to information the user and thus drastically affect the narrative. With the Internal point-ofview, much like the first person viewpoint of traditional narrative, the user is bound to a specific character; be it a digital avatar created according to their choosing, or a character that is heavily involved in the story of the world. The user has a single point of reference that they can control, and use to interact with things. They are limited in time and space 
and cannot roam around freely as they choose within the story world or system of information. As a result the narrative scope tends to be a little more limited, a little more personal, and the user tightly bound to different elements of the world such as being confined to a certain place or only being able to view things in a certain order.

In contrast, in the External point-of-view the player has near unlimited freedom to roam around and possibly control things as they wish. This could range from an impersonal observational ghost, a viewpoint that is not part of the narrative world which they can use to view the narrative, to being an almost omniscient god who can explore every aspect of the world in any order.

\subsubsection{Visual Style}

How the content will be displayed is a separate matter from how it is arranged. Museums tend to be highly visual places with lots of visual information. As detailed Appendix I: Sources of Content, only one exhibit, the Audio Exhibit, out of the eleven exhibit types lacked a visual component as the main focus of the exhibit. Furthermore, only one of those exhibits, the Infowall, had a text based component as its main focus over some other type of visual element be it images, video, artefacts, diorama's etc.

This project seeks to not only generate a narrative to convey the users' experiences in the museum, but to do so in a visual manner. The different ways the narrative could be conveyed visually and their visual style was an important consideration in the design as the structuring of the narrative and form.

In Narrative Visualization: Telling Stories with Data [35], Segel and Heer state that: 
"Choosing the appropriate genre depends on a variety of factors, including the complexity of the data, the complexity of the story, the intended audience, and the intended medium."

They detail seven different genres that they found useful for presenting visual narratives and which offer visual metaphors for interaction. They are: the magazine style, the annotated chart, the partitioned poster, the flow chart, the comic strip, the slide show, and the film/video/animation.

To this end, four of the seven genres were selected by the researchers involved in this project. They were selected because the type of data that a user would collect in a museum would work well with them; they would be very likely to be familiar with the intended audience; and they were possible to create and deliver using the proposed platform (web application). They are:

Magazine Style of presentation tends to feature a long page or series of pages with text, pictures, and interactive frames. The information is presented in a linear manner where the user reads it from top to bottom or start to finish.

Annotated Chart style of presentation is where the data is presented in a graph or chart like manner. There is generally an axis either overt or implied and the user can see the data visualized in a quantified manner. The chart is often annotated which helps convey the key events, conflicts, or characters in the narrative.

Partitioned Poster is similar to the magazine style except it tends to be a single page and the page is partitioned into several different sections or smaller infograpics. This allows the presentation of a variety of data. The viewer also doesn't read it in a linear manner 
like a magazine, but instead explores it by reading the different side panels and looking at the different parts as they choose.

Slide Show the slide show presents a series of images that detail a series of events over time. The images are generally shown chronologically. This method is very good at presenting visual events or actions that gives a sense of the space, what happened in it, and who was involved.

\subsection{The Four Narrative Visualizations}

It is from the information presented above that the concepts for our four different narrative visualizations were designed. The interactivity and points-of-view in the works of Marie-Laure Ryan shaped the form that the narrative would take and the narrative genres presented in the work of Segel and Heer heavily influenced the style. They are not the only narrative visualizations that could be designed. A variety of other visualization methods and styles could be designed with the other unselected narrative visualization genres such as one in the style of a comic strip or one in the style of an interactive animation. Only four were created for this research study to narrow the scope of the usability testing.

The conceptual designs for the four narrative visualization styles that this thesis sought to evaluate are briefly detailed as follows. Their implementations are fully discussed in the next chapter.

Categorical (External, Exploratory; partitioned poster genre): In this narrative visualization, the users' experiences in the museum are presented from a removed 
perspective where the presentation is impersonal. There is little to connect them to time and place and they can explore their experiences in a freeform manner. Visually, this takes the form of presenting the user with information partitioned into a series of pages or sections, all arranged in a categorical fashion by scientific subject like a partitioned poster. The user can explore each subject by exploring the various sections.

Slideshow (External, ontological; slideshow genre): In this narrative visualization, the users' experiences in the museum are again presented from a removed perspective where the presentation is impersonal. However, the users are able to view them in a way that gives a sense of what they decided to see in the museum. The slideshow genre fits this method of narration very well where the data is presented in loose chronological sequence and we get an idea of what the user decided to see and do, but not much personal detail.

Dramatic (Internal, Exploratory; magazine genre): In this narrative visualization, the users experiences in the museum are presented from a personal perspective. Their experiences are presented in a free flowing personal manner that tries to show what they explored and how they felt about it, rather than a strict sequence of events or places. Visually this would take the form of a virtual magazine like scrapbook of what the user experienced in the museum; the content would be heavily personalized with comments about what was seen. In this style, the users' interaction and exploration with the museum is apparent, as if the user is a character within the story that is being presented.

Sequential (Internal, ontological; annotated chart genre): In this narrative visualization, the users' experiences in the museum are presented from a single vantage 
point, albeit an impersonal perspective. What the user decided to see and visit in the museum is presented in a way that gives a sense of the time and place of their happening. Visually, this would take the form of a map with the annotated exhibits and the user's path through the museum showing their journey. This annotated map would be visually and functionally similar to the annotated chart genre although lack an overt axis or strict grid. 


\section{Chapter 4: Prototype Design and Implementation}

\subsection{User Scenarios}

To begin the design process of a system to implement the four visual narratives, two user scenarios were written to describe how users of the system would use the imagined final implementation. User Scenarios are useful tools because they give a detailed account of the user activities when using the system. They can help verify if the design would make sense to the user and explain what user needs the design answers [39].

\subsubsection{Scenario 1: Visiting a Museum and Constructing a Visual Narrative}

The first user scenario revolves around a user visiting a museum. In this scenario the user is building a narrative visualization based upon what she experienced firsthand in the museum. The two main goals of this user scenario are construction and reflection.

Amanda decides to visit the Museum of Nature with her family. She goes on a Saturday and takes her children through all the exhibits in the Mammals section of the museum. They take photos with their cameras and her children interact with a couple of the interactive exhibits that have simple buttons to press and knobs to turn. They also play with the interactive touch screen quizzes and spend a long time looking at and talking about the life sized animals in various dioramas. As she explores the museum, the museum app on her smartphone tags pictures that she takes, saves audio and video that she records, notes the exhibits she visits, and helps prompt and guide her along her itinerary. At the end of the 
day when she gets home she can use it to choose from several visualizations that will offer an interactive presentation of her experience of that day.

\subsubsection{Scenario 2: Sharing a Visual Narrative with Others}

The second user scenario revolves around a user sharing their Visual Narrative with others. In this scenario the user is looking at visualizations of a friend or family member full of things they haven't experienced firsthand. The two main goals of this user scenario are sharing and communication.

Reginald wants to visit the Museum of Nature while on vacation. He doesn't know much about it and goes to its website and looks at a map of its floor plan. This gives him an idea of what's inside and where everything is but not a great idea of what the experience is like. He sees that he is able to browse the narrative visualizations of others. He decides to view several to get a better idea of what the exhibits are like to actually see in person and the different things people saw. He sees that he can plan a tour using a web application on the website and then follow it with an app on his smartphone. The app would also allow him to create a visualization of his own. A few weeks later, when he returns home his family want to hear about his trip. He shows them the narrative visualization that he made and walks them through his trip. 


\subsection{Prototype and System Scope}

\subsubsection{Prototyping}

After reviewing the scope of the project and the resources and time needed, it was decided that a full final version of the system would not be implemented for usability testing. Additionally, the development of a prototype over a final version was the next logical step in the process of the entire project. The MEseum study [8] had performed proof of concept research to see how well the idea of the system would be received and what features users wanted. This project continues that research by focusing on a subset of those features, the timeline.

Almost all interaction design includes some form of iteration, and mock-ups and prototypes are very useful in the design process to help evaluate the design and see if there is room for improvement [35]. Thus a prototype was constructed in order to expand the timeline feature of the MEseum study [8] and to answer the research hypotheses.

\subsubsection{System Scope}

While the user scenarios describe functionality that would be desired in the final version, because a prototype was being built instead of a final version, the scope of the functionality is less than it would be in a final version as described in the scenarios. Specifically, the user did not visit a museum and instead it was decided that the museum would be simulated. 
To this end, the functionality of the prototype was divided into two main sections, a Virtual Museum that the user could use to explore the museum and gather data, and a Narrative Visualization Constructor.

\subsection{Virtual Museum}

The virtual museum is a tool that the user could use to explore a virtual museum and collect data related to their simulated museum trip. This data would be used to create the narrative visualizations.

In the final non-simulated version, parts of this tool would still exist where it would use the museum map and itinerary functionalities to help guide a user along the tour that they have created. In this prototype version, because the museum visit is simulated there is additional functionality related to viewing exhibits and artefacts. Overall the core functionality of the virtual museum is as follows:

- Planning and Guiding: The virtual museum lets the user plan and follow a trip through the museum.

- Exploring: The virtual museum lets the user learn, interact and explore various exhibits and experience what the museum has to offer.

- Collecting: The user is able to interact with artefacts in the museum and the system gathers data on these exhibits and the user's interactions.

The virtual museum's structure is comprised of two parts; a museum explorer which prominently features a floor map of the museum and works like a guidance system; and 
an exhibit viewer which lets a user view and interact with a virtual exhibit and the artefacts contained there within.

\subsubsection{Museum Explorer}

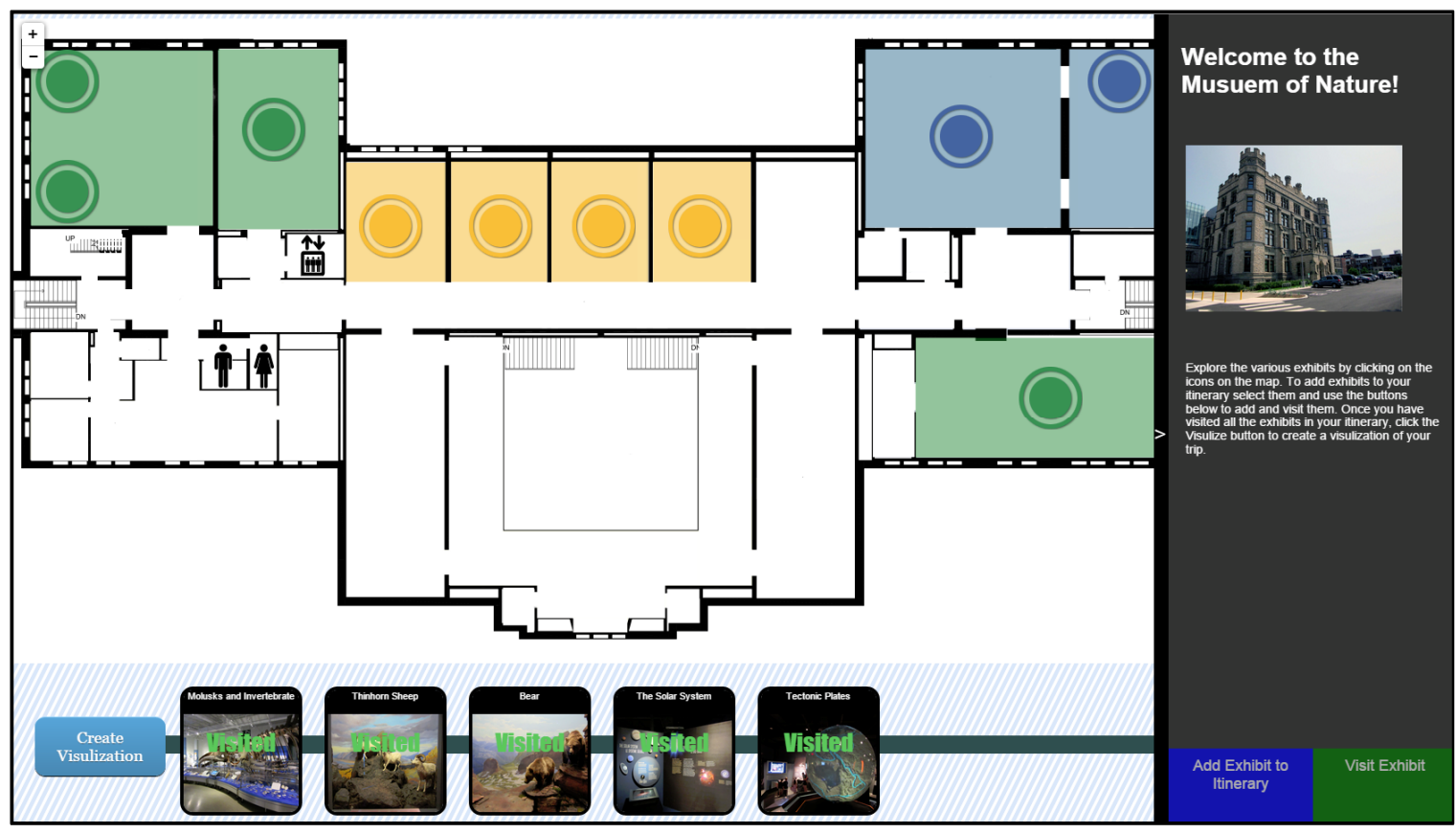

Figure 3. Museum Explorer

The Museum Explorer is a tool that the user can use to explore the museum. It is similar in function to the virtual museum in the previous MEseum project. Where a user is presented with a view of the museum floor plan. The main differences is that the MEseum system had more way-finding, path planning, and 'check-in' functionality and different map layers to show the other users present in the museum. These features were not incorporated into this project, as they were not relevant to the process of constructing a narrative visualization. Instead, a simpler system of making and following an itinerary was used. The user can click on an exhibit to view information about it and add it to an itinerary of exhibits that they want to visit. This itinerary is updated as they visit different 
exhibits. The main functionality of the Museum Explorer is divided into three main user interface (UI) features:

The Map is in the center area of the screen. The user can scroll around the map by clicking and dragging and by using the mouse wheel to zoom in and out. The map contains the floor map of the musuem with icons signifing exhibits. The icons can be clicked on and the side panel will pop out.

The Side Panel contains pictures of the exhibit, some information on it, and two important buttons at the bottom that deal with the exhibit. The first is to add/remove the exhibit from the itnierary across the bottom of the screen. The second is to visit the exhibit. If the visit buton is clicked the user is brought to the exhibit viewer detailed in the next section of this document.

The Itinerary is a shelf along the bottom of the screen with a button at the endused to create a visulizaiton. The user can add and remove exhibits from the itnerary using the add/remove button from the side panel. When clicking on an exhibit on the itinerary shelf, it will be selected and highlighted on the map and the side panel will display related information. Exhibits on the itinerary shelf have two states, visited and unvisited. If the user visits an exhibt in their itinerary, it's status will change to visited. Once all the exhibits in the shelf have been visited (or the univisted ones removed) the user can click the visulize button which will end their trip and bring them to the Narrative Visuilzation Generator. There they can use the Narrative Visuilzation Generator to visulize the narrative of their visist thus far. 


\subsubsection{Exhibit Viewer}

This is a tool that allows the user to explore an exhibit. It lets the user see and interact with various artefacts in the museum and allows the user to collect data overtly, such as allowing pictures to be taken, or covertly through the system calculating how long they spent at each exhibit. This tool is primarily developed for simulation purposes. The main functionality of Exhibit Viewer is divided into three main UI features:

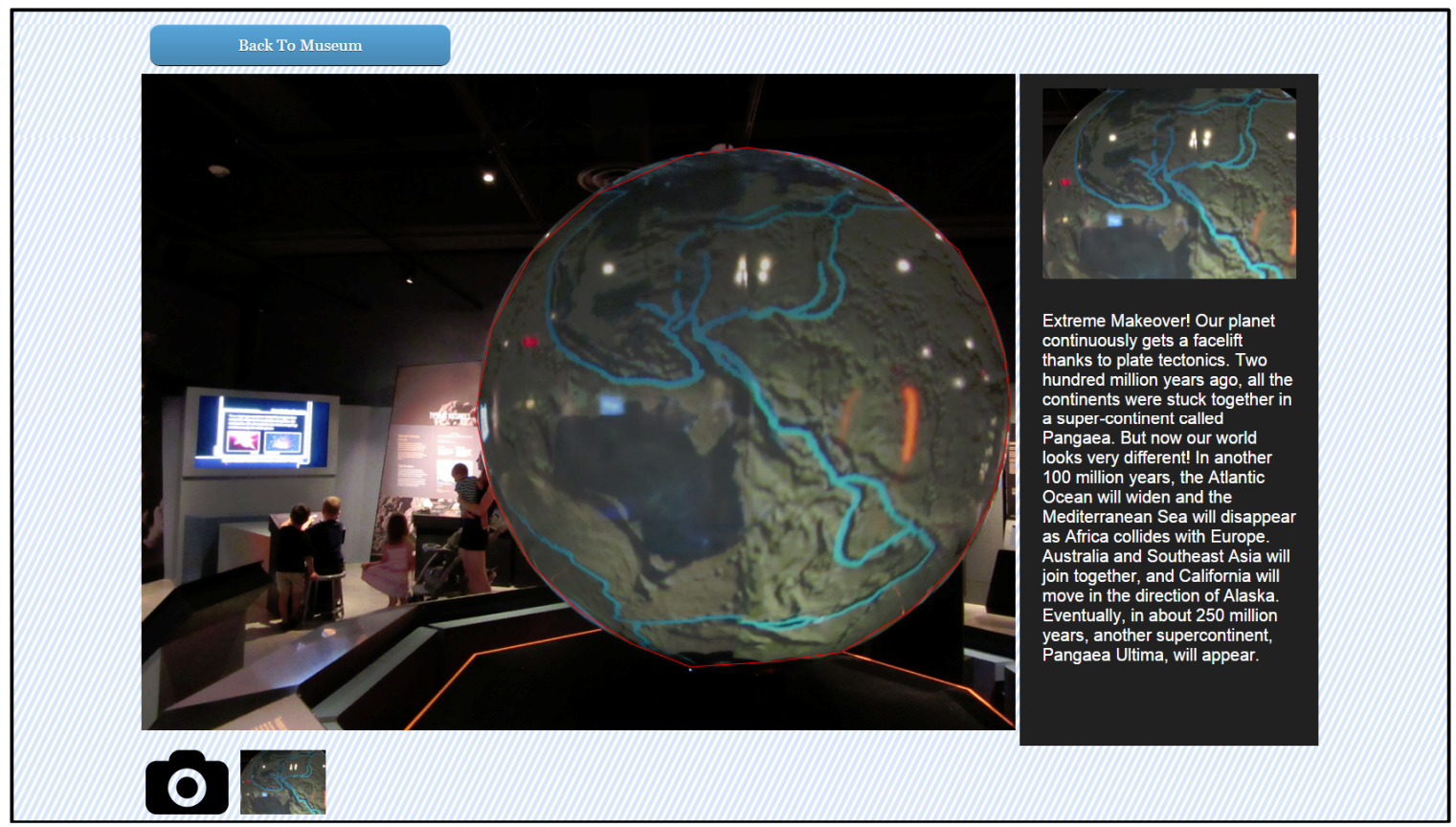

Figure 4. The Exhibit Viewer

The Viewing Area is very similar to the main area of the Musuem Generator. A large establishing shot of the musuem exhibit is presented to the user. They can mouse over and click on different parts of the exhibit in order to read information about the artefacts. When they do click on an artefact in the exhibit it is highlighted as selected and information about it appears on the side panel.

The Side Panel displays information about the artefact. 
The Camera Shelf is a little shelf at the bottom with a camera icon. Whenever an artefact is selected and the camera icon is clicked a pop-up appears of a picture of the artefact. They can add a comment to the picture if they choose to and then close the popup. Once the popup is closed a little thumbnail image of the artefact is added to the camera shelf.

\subsection{Narrative Visualization Constructor}

The main purpose of the narrative visualization constructor is to allow users to review their trip and create narrative visualizations that that they can present to others or reflect upon and remember their experiences. The final version of this tool would be similar to what exists in the prototype and the core functionality and design would be the same. The functionality would simply be more robust offering more options and greater flexibility to the user. Part of the reason the prototype was developed over a final version was to get feedback from the users about how the narrative visualizations so the designs of them could be expanded into a final form.

This core functionality aligns with the overall goals of the system, with two of them being tied to specific functionality in the prototype. Communication is part of the overall visual design and usability testing to make sure the functionality is easy to use and bug free, and reflection is not a functionality in of itself but something that is prompted by the system. Overall the core functionalities are as follows:

- Construct: The narrative visualization constructor presents the user with an overview of their trip that lets them review it in a concise manner and decide what content they want to keep and what content they want to discard. They then can 
select from several different visualizations and create a personalized visual narrative of their trip.

- Share: The narrative visualization constructor creates visualizations in a presentable format that the user can share with others.

Data visualization tools are often built to allow someone to analyze and make sense of data, and then present these findings [28]. In accordance, the narrative visualization constructor is comprised of two parts: a selection stage that lets a user view their trip and select a visual narrative; and four visual narratives that they can chose from to present their trip.

\subsubsection{Selection Stage}

Step 1: View your Visit
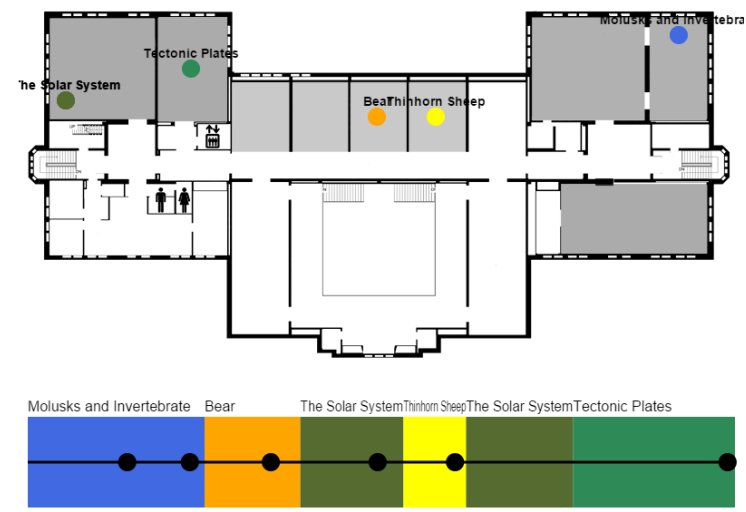

Figure 5. The Selection Stage

The selection stage allows users to review and analyze their visit and select a visual narrative to present their data. It does this primarily through an overview of what exhibits 
the user visited and where they were, how long they stayed at each place, and what content they collected.

This stage is not concerned with providing a lot of detail on the trip. It aims at providing just enough so that the user can remember what they did and select what exhibits they want to retain for presentation and what they want to discard. They do this through the following UI elements:

Map like in the virtual museum, a map is displayed centrally. However, unlike that map, this one is static and simply displays the exhibits the user visited in a colour coded fashion.

Timeline is a simple colour coded representation of the visit displayed below the map. Each block corresponds with a visit at a particular exhibit and the length of each block indicates how long the user spent at that exhibit. Across the entire timeline is a horizontal bar with small little circles on it. The circles correspond to points in time when the user took a picture or saved a note at that exhibit. The user can hover over the circles to see exactly what they saved and check the circle in order to retain the data for the next step. 


\section{Step 2: Select Exhibits to Include in the Visulization}

Now, that you have reviwed your tip, please select which exhibisis you want to include in the visulization of your trip. The visulization will serve as a reminder of your trip that you
can reflect upon and share with others. It will include the pictures you have taken on your trip and the notes you have mast

$\checkmark$ Molusks and Invertebrate $\square$ Bear $\square$ The Solar System Thinhorn Sheep $\square$ Tectonic

Step 3: Select Style of Visulization

\section{Slideshow}

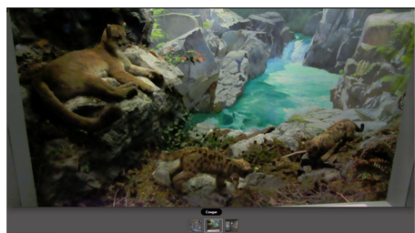

Figure 6. The Selection Stage (continued)

Exhibit Selection: In the section labelled "Step 2: Select Exhibits to Include in the Visualization" there is a series of labelled checkboxes that correspond to the exhibits the user visited. The user can select which exhibits they want to include in their narrative visualization or discard them.

Visualization Style: This last step is concerned with presenting the four narrative visualizations that the user can select as means of presenting their experiences in the museum. Each narrative visualization is briefly described with an accompanying picture of what it looks like and a button to generate it.

\subsubsection{Visual Narrative 1: Slideshow}

This visualization is a rather simple one. It's meant to mimic the existing method of displaying pictures in a slideshow that is commonly used as a presentation means. It's not 
really trying or meant to be all that interesting or innovative. It was included to serve as a comparison to the other visualization methods.

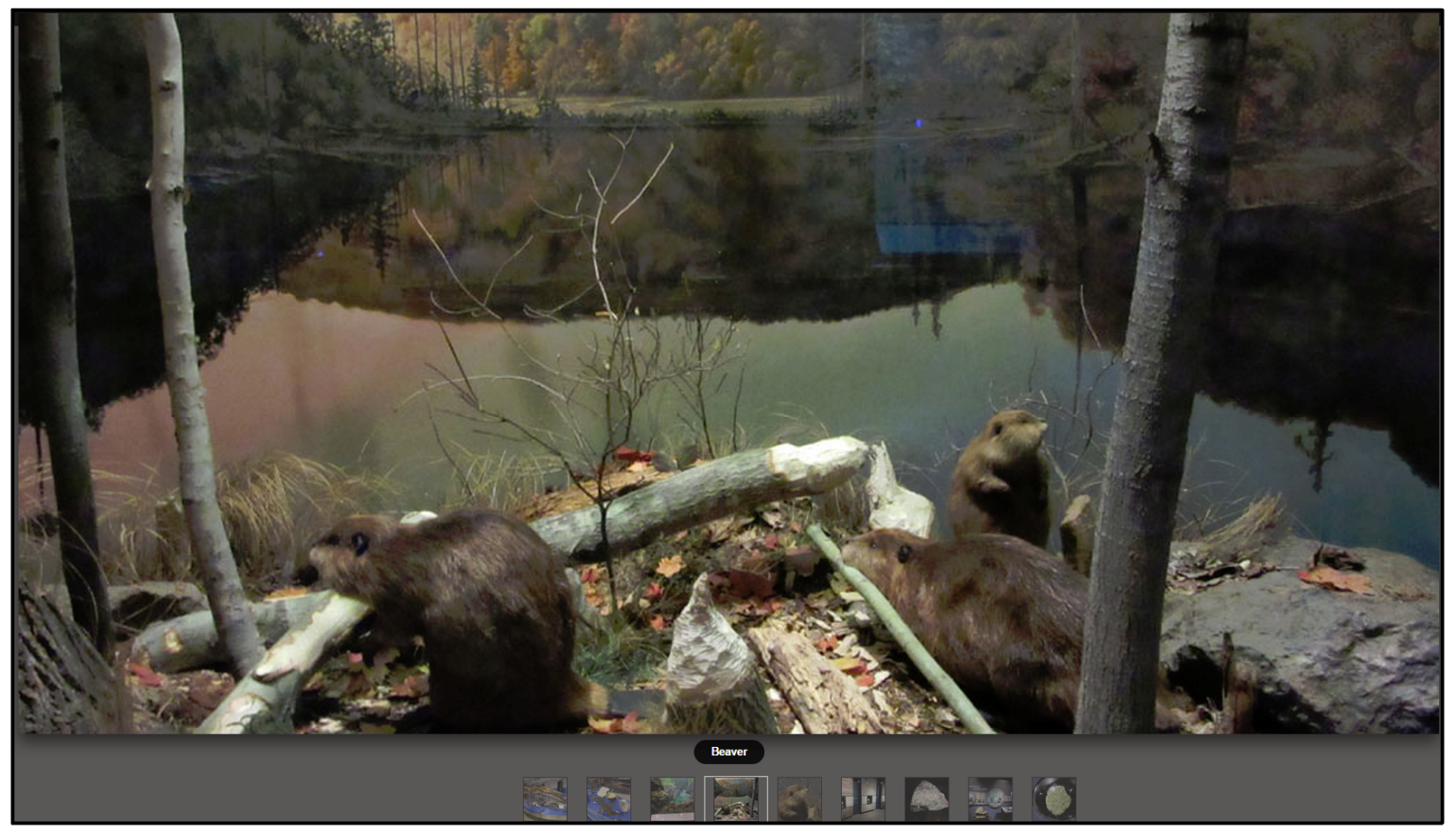

Figure 7. The Slideshow Narrative Visualization

Large View Area: The pictures are displayed very prominently, almost full screen. They fade into each other as the slide show automatically plays and rotates through them all.

Slideshow Queue: There is a small queue underneath the viewing area that displays all the photos along with the comment or title associated with the photo currently being displayed. The user can click on a specific photo in order to cycle to it immediately.

\subsubsection{Visual Narrative 2: Categorical}

This visualization is focused around giving a clear sense of the information learned. It presents its information impersonally as more of a record of what the user learned and visited. Information is arranged in a scientific or categorical sense by the topic of the 
material and the user can freely browse and explore what they visited without a relational sense of time or place. It consists of a few main interface features:

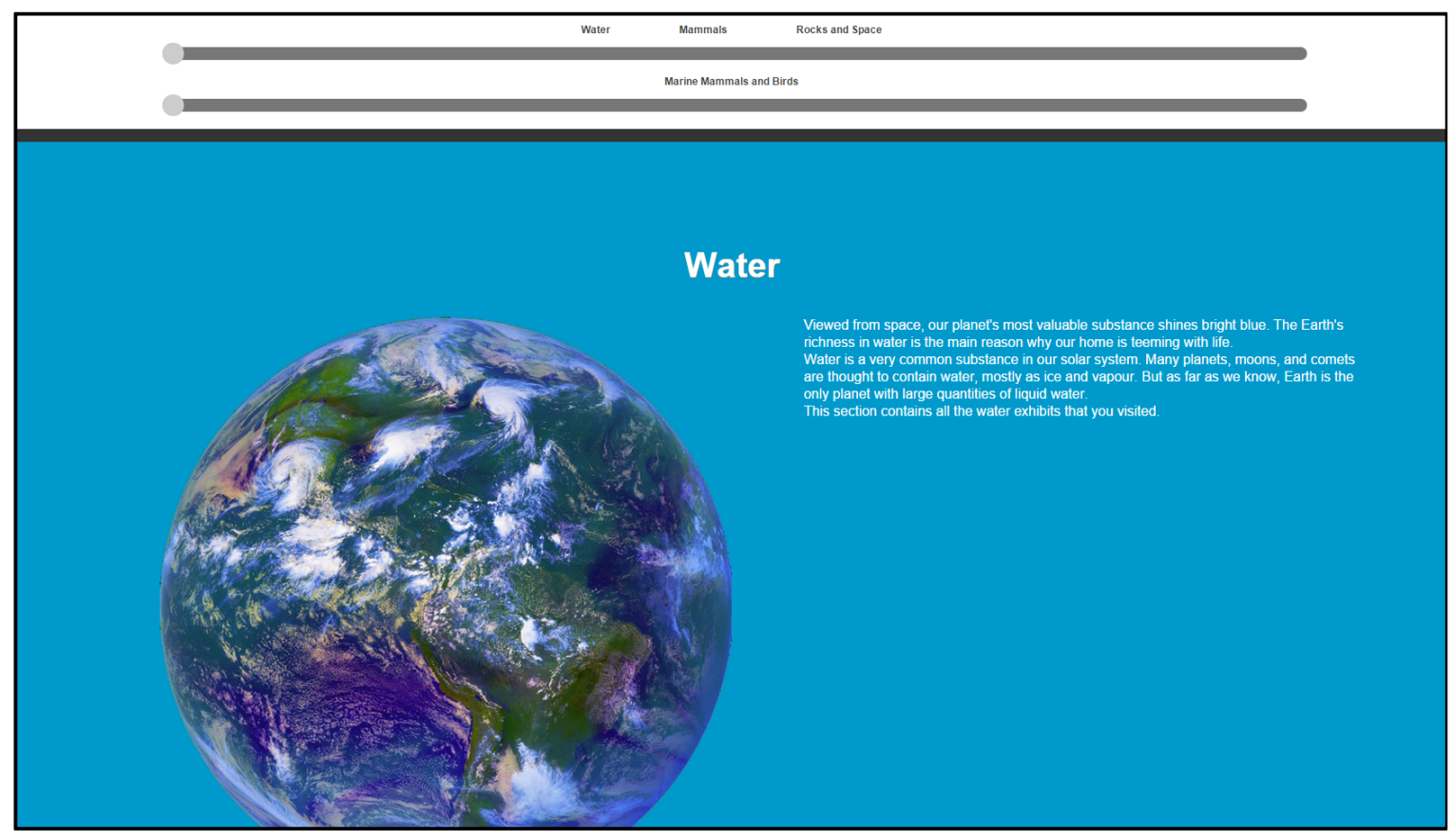

Figure 8. The Categorical Narrative Visualization (Title Page).

Title Page: Each category has a title page. It has a large image and paragraph that describes the theme of the exhibits that were in that section of the museum. It's meant to give an overview of the category. The background is colour coded to the exhibit category and the scrollable menus have animations that slide the content onto the page.

Menu Bars: The menu bars located at the top allow the user to scroll through two things. The first is the categories of the exhibits the user visited at the museum, such as the mammal exhibits, the mineral exhibits, etc. The second menu bar is used to scroll between the specific visits within those categories. Overall in this manner, the type and category of information they viewed at the museum is all laid out for them and they can gain an understanding of how it is all scientifically related and categorized. 


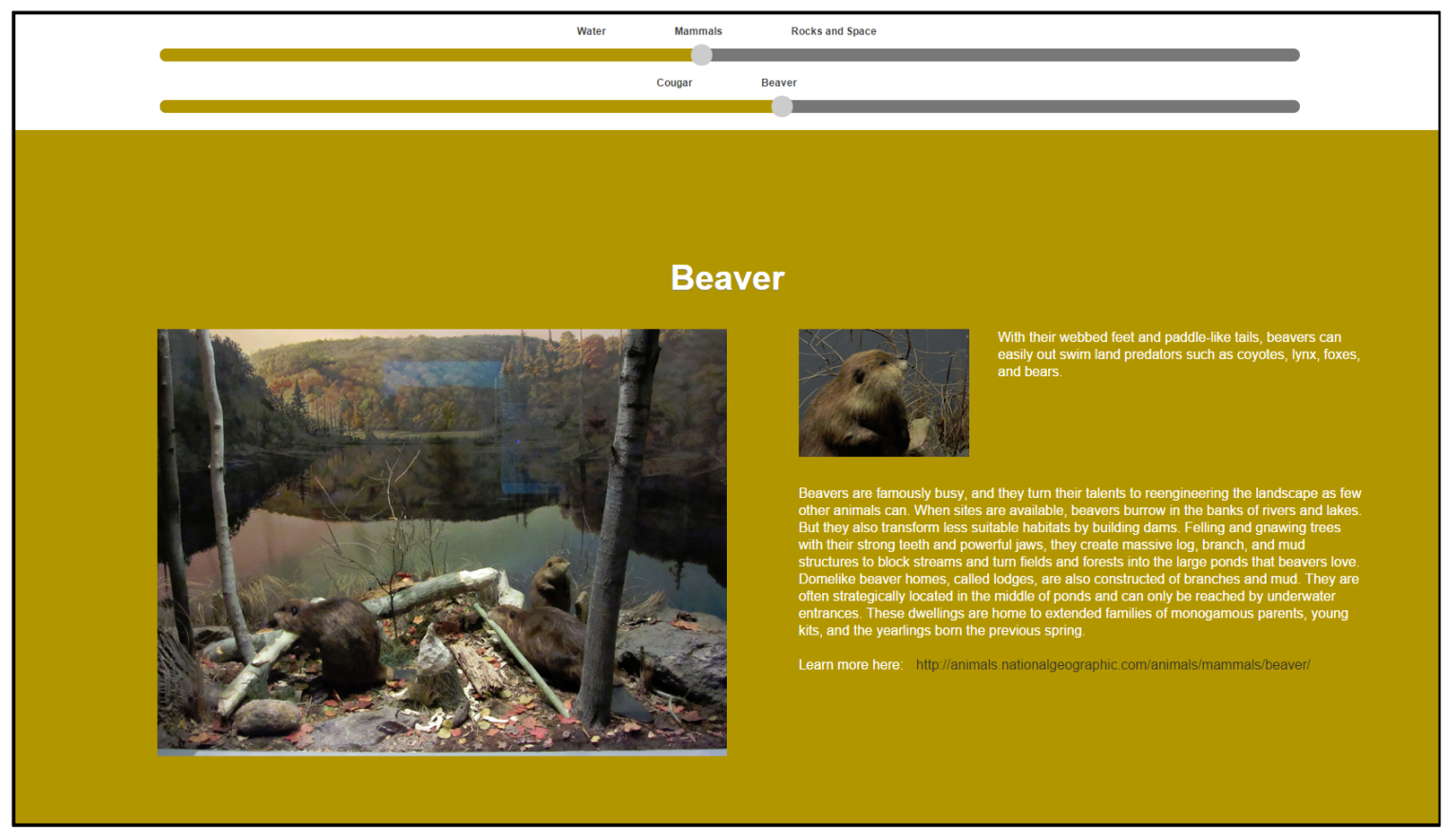

Figure 9. The Categorical Narrative Visualization (Exhibit Page).

Exhibits: Each exhibit is presented in an area the size of the screen. An establishing shot of the exhibit is shown along with pictures of artefacts in the exhibit that the user took. Information about the artefacts is displayed alongside them and information about the exhibit is displayed below. It is all laid out in a magazine style layout. This narrative visualization is focused on learning and so is impersonal in presentation where the users comments about what they saw aren't displayed. Instead some information from another source outside the museum is presented along with a weblink to that source encouraging the viewer to learn more.

\subsubsection{Visual Narrative 3: Sequential}

This visualization is focused around giving a sense of time and place. Each exhibit is presented like a node on the overall museum floor plan. The user can follow the path they took and view each exhibit they saw. It consists of two main interface features: 


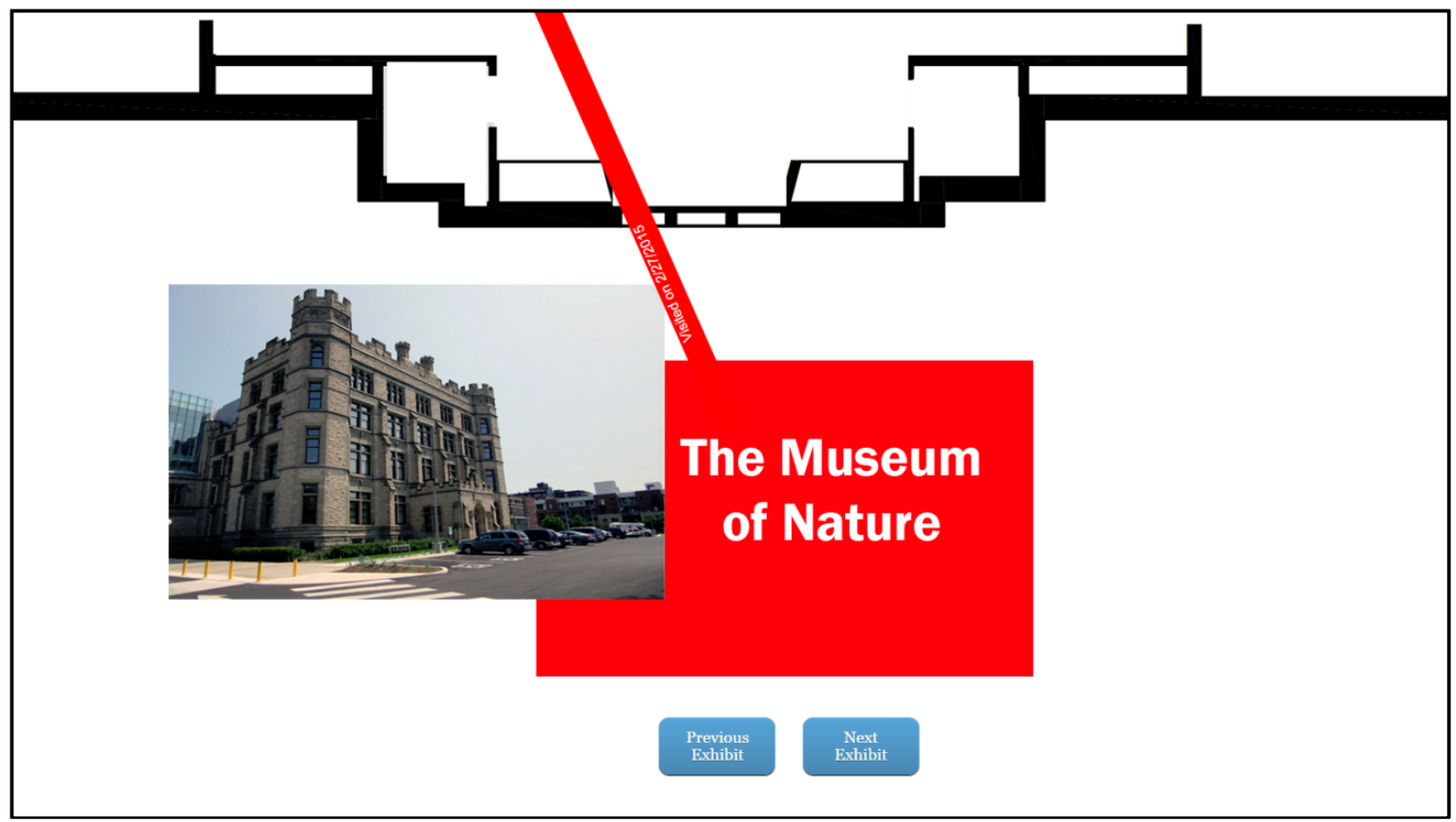

Figure 10. The Sequential Narrative Visualization (Starting Position).

Users Path: The path the user took from exhibit to exhibit is displayed with the time the user arrived at and left each exhibit. They can quickly use the 'Previous Exhibit' and 'Next Exhibit' buttons to quickly follow this path and go from exhibit to exhibit. 


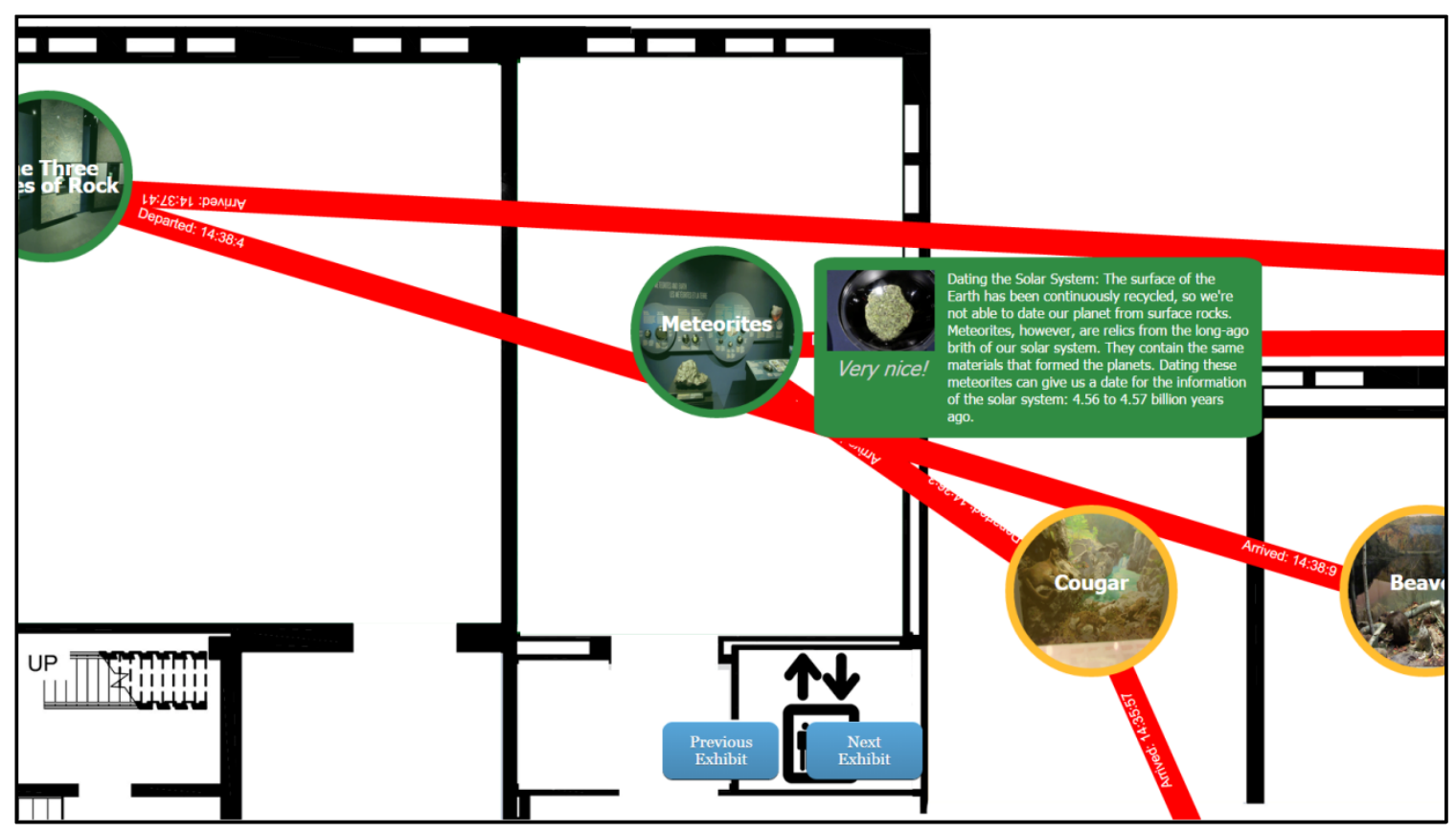

Figure 11. The Sequential Narrative Visualization (Viewing an Exhibit).

Radial Menu: Each museum exhibit is displayed as a node. If the user clicks on it a radial menu of nodes is displayed around the central node. Each child node is a picture of an artefact in the exhibit that they took. If they click on an artefact node then the information about the artefact is displayed.

\subsubsection{Visual Narrative 4: Dramatic}

This narrative visualization is focused around giving a clear sense of the user's personal experiences visiting the museum. There is a sense of linear narrative to it where the user scrolls and what they saw in the museum is presented to them in chronological manner. It consists of a few main interface features: 


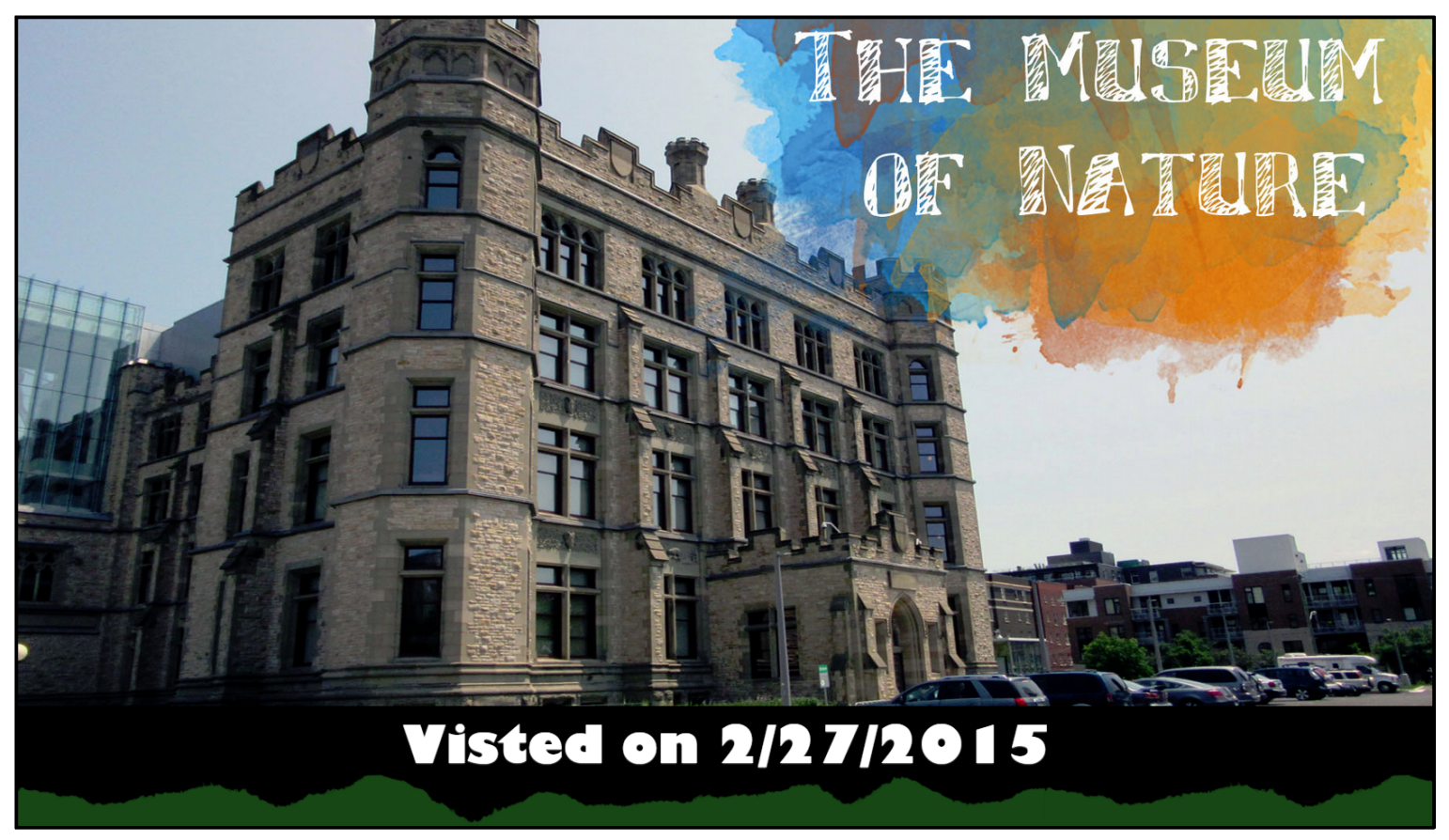

Figure 12. The Dramatic Narrative Visualization (Start)

Title Slides: The part of the page is a title page prominently displayed and shows the name of the museum and a picture of the entrance. It serves to give a sense of place and context to what follows. The date the user visited the museum is also displayed. 


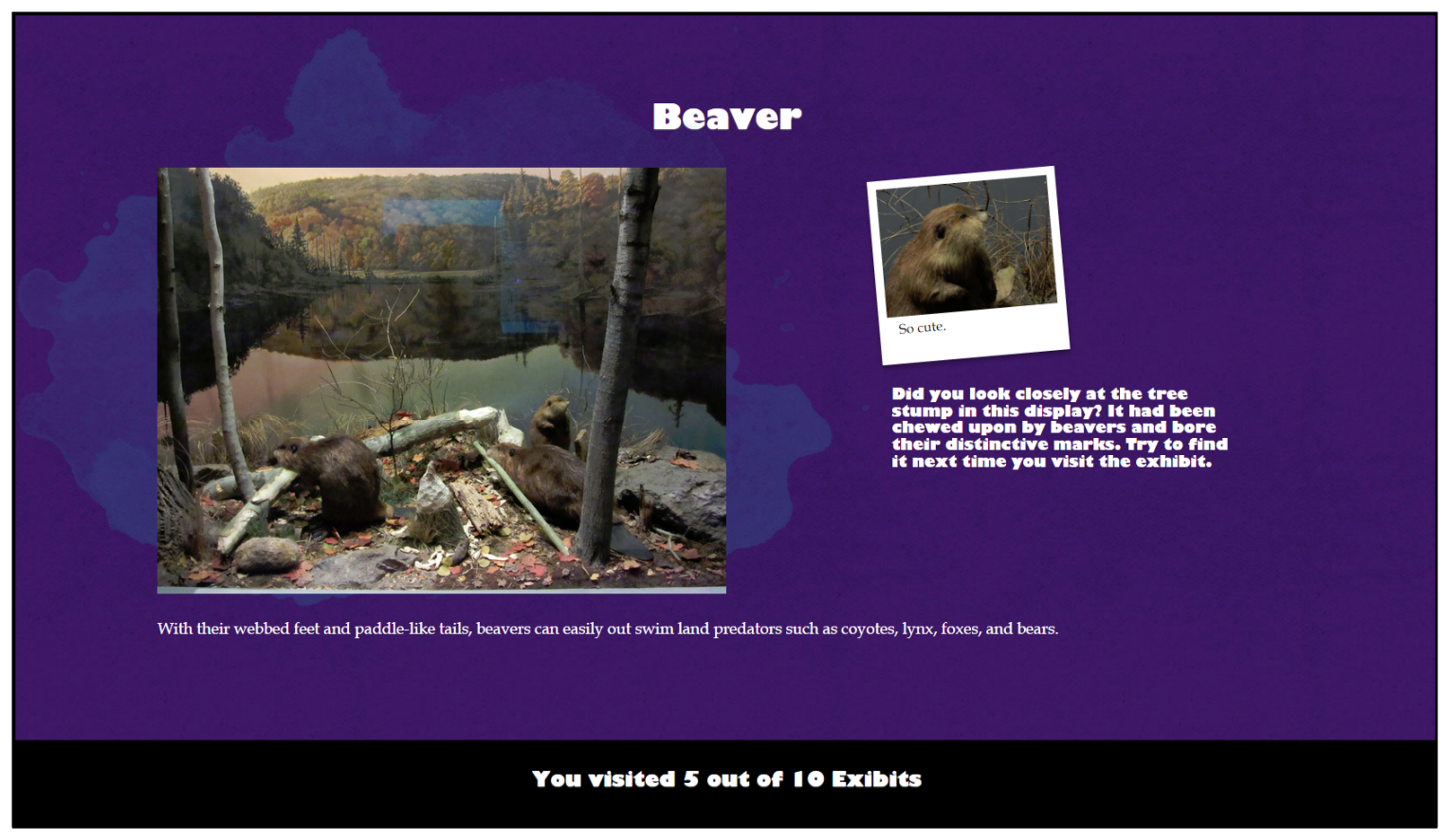

Figure 13. The Dramatic Narrative Visualization (Viewing an Exhibit)

Scrapbook Page: The exhibits the user saw are laid out like in a scrapbook like format all down the page, one after the other. The background is textured and the font playful. Any pictures the user took are displayed like photographs with their comments written on them. Information about the exhibit is kept to a minimum to keep the presentation personal. 


\section{Meteerites}

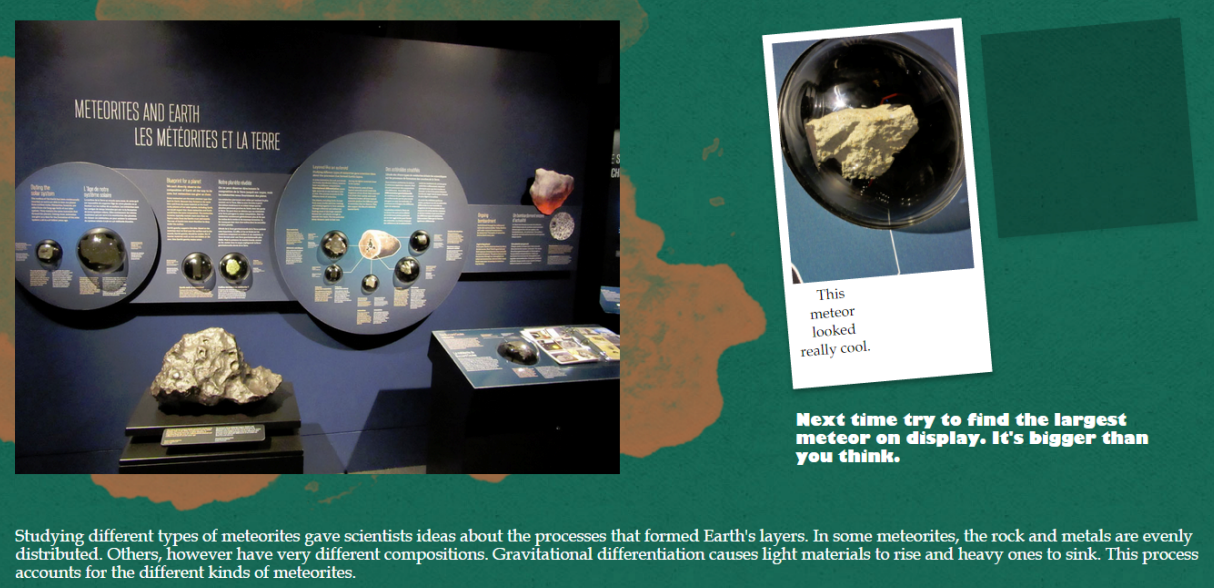

Figure 14. The Dramatic Narrative Visualization (Viewing another Exhibit)

Museum Comments: Depending on the artefacts that the user took pictures of the system will make different comments about what they saw. For example in the above exhibit the comment is "Next time try to find the largest meteor on display. It's bigger than you think." These comments are meant to create a sense of interaction with the user where the museum itself is seen as a character and their journey through it as an exploration.

Unseen Exhibits: If the user missed taking pictures of any prominent exhibits 'blank' squares will be displayed next to the polaroids of the pictures they took. These blank squares are meant to give a sense of what the user saw on their visit as well as what they did not see. 


\subsection{Technology Used and Software Development}

There were a total of seven webpages in the prototype. The virtual museum page contained all the functionality of the virtual museum as described in Section 4.3. Whenever the user wanted to visit an exhibit, they would click a 'Visit Exhibit' button to go to the exhibit explorer page. It would generate the exhibit and the user could explore it and collect data.

Once the user was ready to build a narrative visualization they would click on a 'Visualize' button which would take them to the narrative visualization constructor as described in Section 4.4. On that page, there were buttons that linked to the various visualization pages that would generate the visualizations.

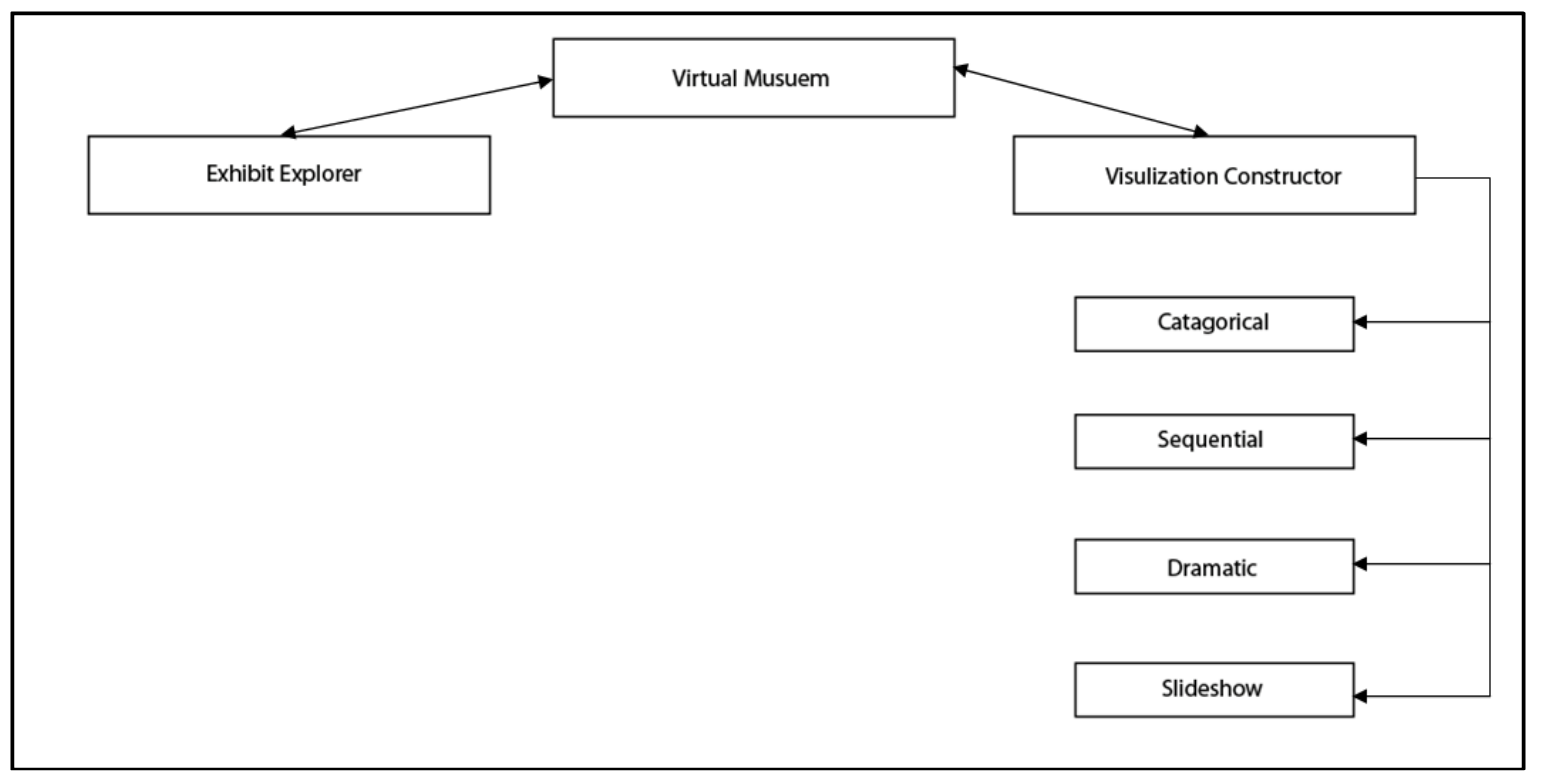

Figure 15. Sitemap of Pages in Prototype

Several web technologies were used in constructing the prototype including PHP, HTML5, Javascript, MySQL, and CSS. Overall the system used a model-view-controller setup and the architecture for each page was similar. 
Once the PHP file was loaded a PHP header would load the correct data from the database. Data, such as how many exhibits there were in the museum, how many artefacts there were, what pictures went with what artefacts, what exhibits the user had visited, etc.

The PHP header would load the data and then the HTML body would arrange it into a visual presentation and CSS would style the page. If the user clicked on any interactive elements in the page JavaScript functions would be called to perform various actions. Then, once they wanted to navigate away from the page, JavaScript would write a bunch of variables to the database in order to keep track of user actions and changes. This would allow data to persist across pages.

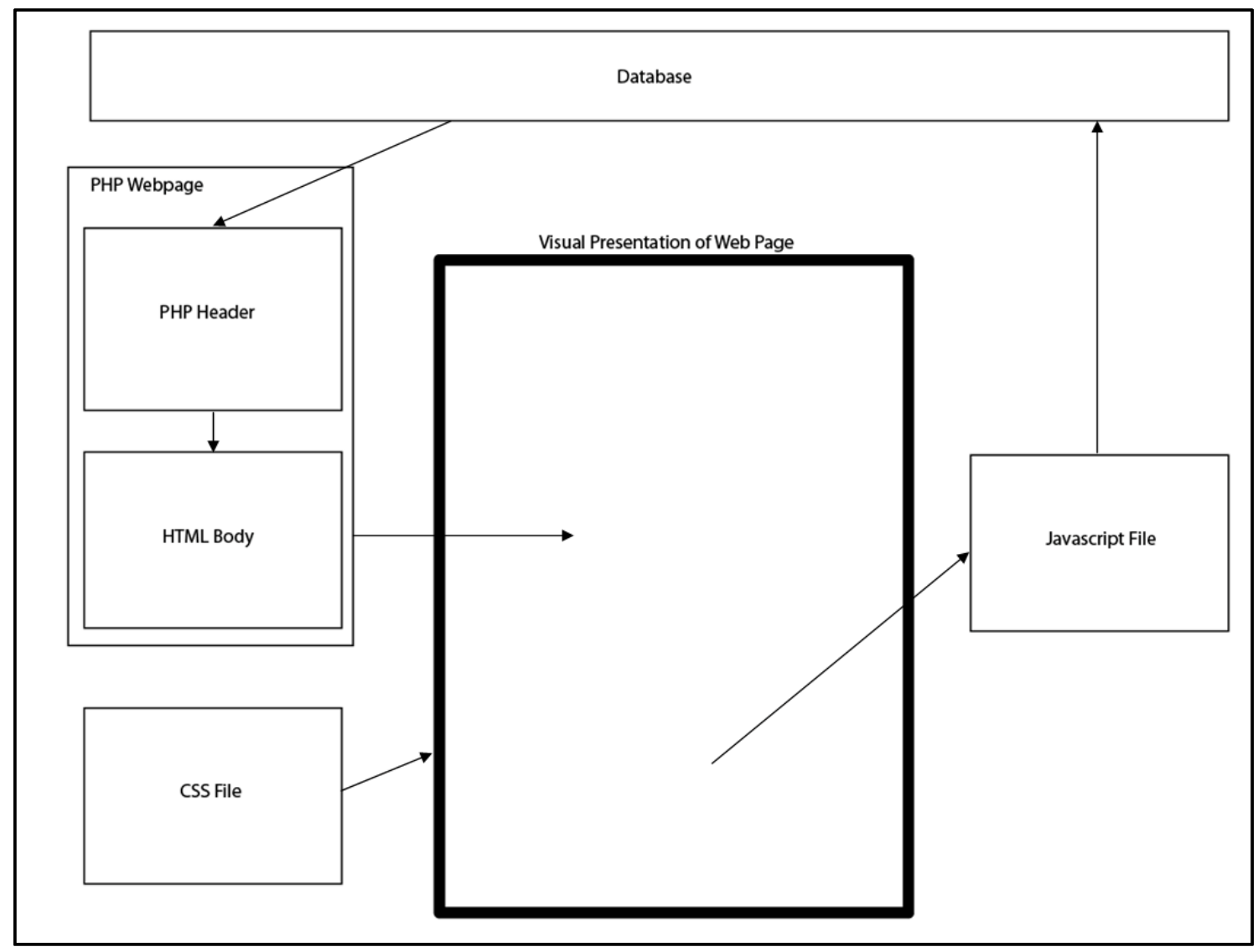

Figure 16. Page Architecture 
A number of JavaScript libraries were used to assist interaction. They are as follows:

jQuery (http://jquery.com/ ): For easily using JavaScript to create animated and dynamic content, e.g. creating slide-in views, or changing content based on button presses.

fancyBox (http://fancyapps.com/fancybox/): To provide the image popup when you took a picture and for the functionality of the Slideshow visualization.

maphilight (http://davidlynch.org/projects/maphilight/docs/): To highlight the hotspots in the exhibits image-maps.

Leaflet (http://leafletjs.com/): To provide functionality for the scrollable and interactive museum floor plan map.

MapTiler (http://www.maptiler.com/): To cut up the image of the museum floor plan into a tileable map for Leaflet to use.

EaselJS (http://www.createjs.com/\#!/EaselJS): To create the map in the Narrative Visualization stage. Including visited exhibits locations and the horizontal bar graph of the users trip that displayed which exhibits they had been in and for how long and what data they had collected as points along it.

\subsection{Development Stages}

The prototype went through several different stages of development. These stages didn't really have any formal names and were marked when the development of a core set of functionality had been completed. Throughout these stages the design and look of the 
prototype was refined in an incremental cyclic process where at each cycle new features were added and previous ones were revised.

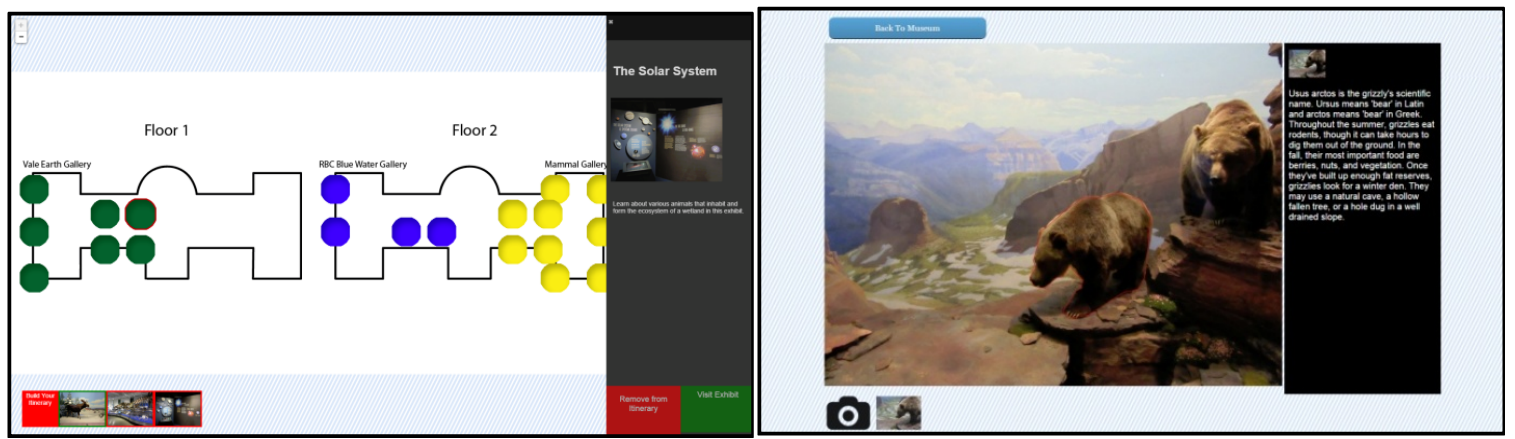

Figure 17. Stage 1 of Development

Stage 1 marked the general development of the museum map, itinerary, and exhibit functionalities. The interface was very rough looking and incomplete.

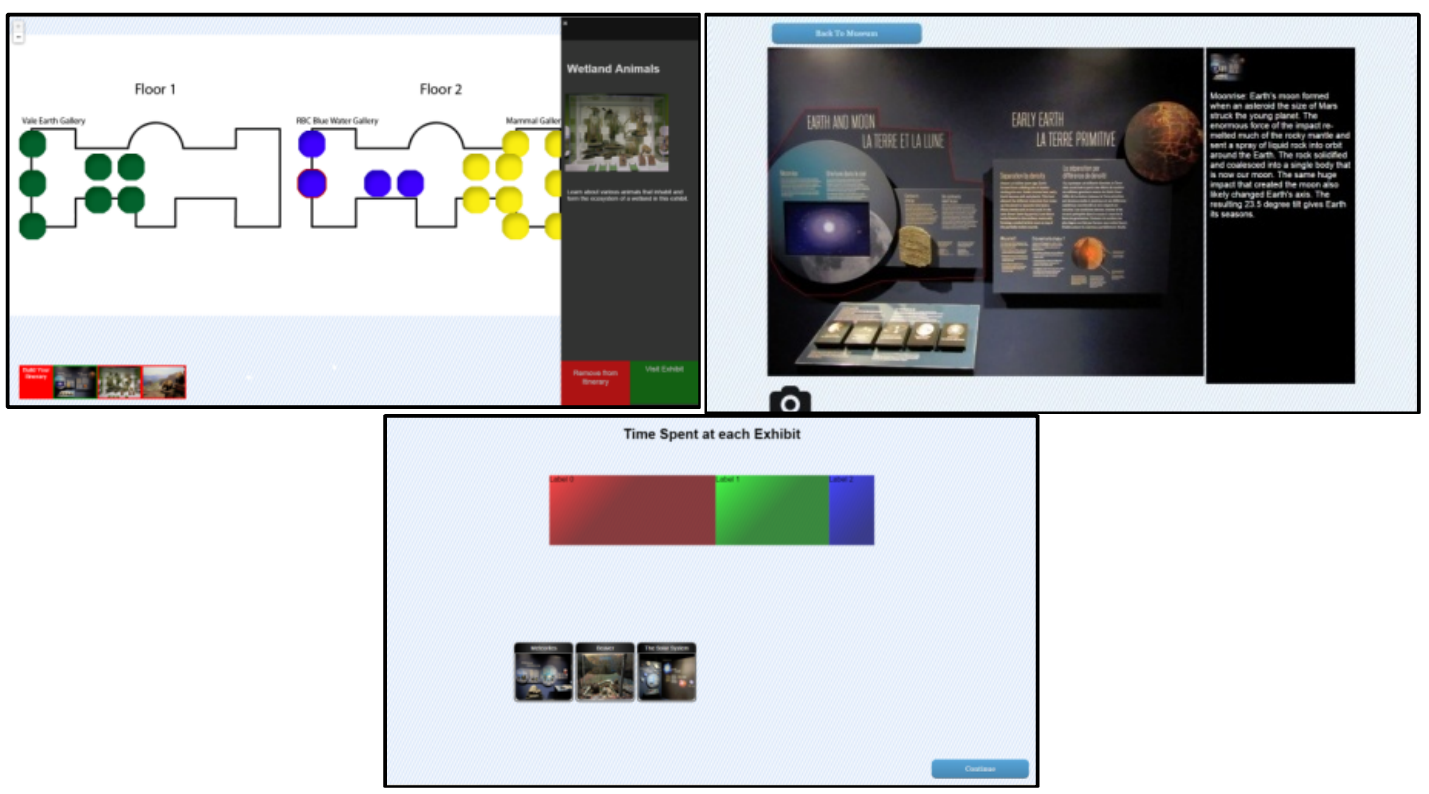

Figure 18. Stage 2 of Development

Stage 2 marked the beginning of the development of the visualization functionalities. The first part of that stage was beginning to be developed as seen in a very early picture of it. 
Two tests were carried out at this point. First was a very informal testing with expert users. They had never seen the system and tested the museum map and museum exhibit functionalities to try and discover usability issues (of which there were plenty). They were then presented with mock-ups of the narrative visualizations with a description of how they functioned. This informal presentation was followed by a questionnaire.

The purpose of the informal presentation and questionnaire was to solicit feedback on the theme and focus of each narrative visualization, and the general design of it.

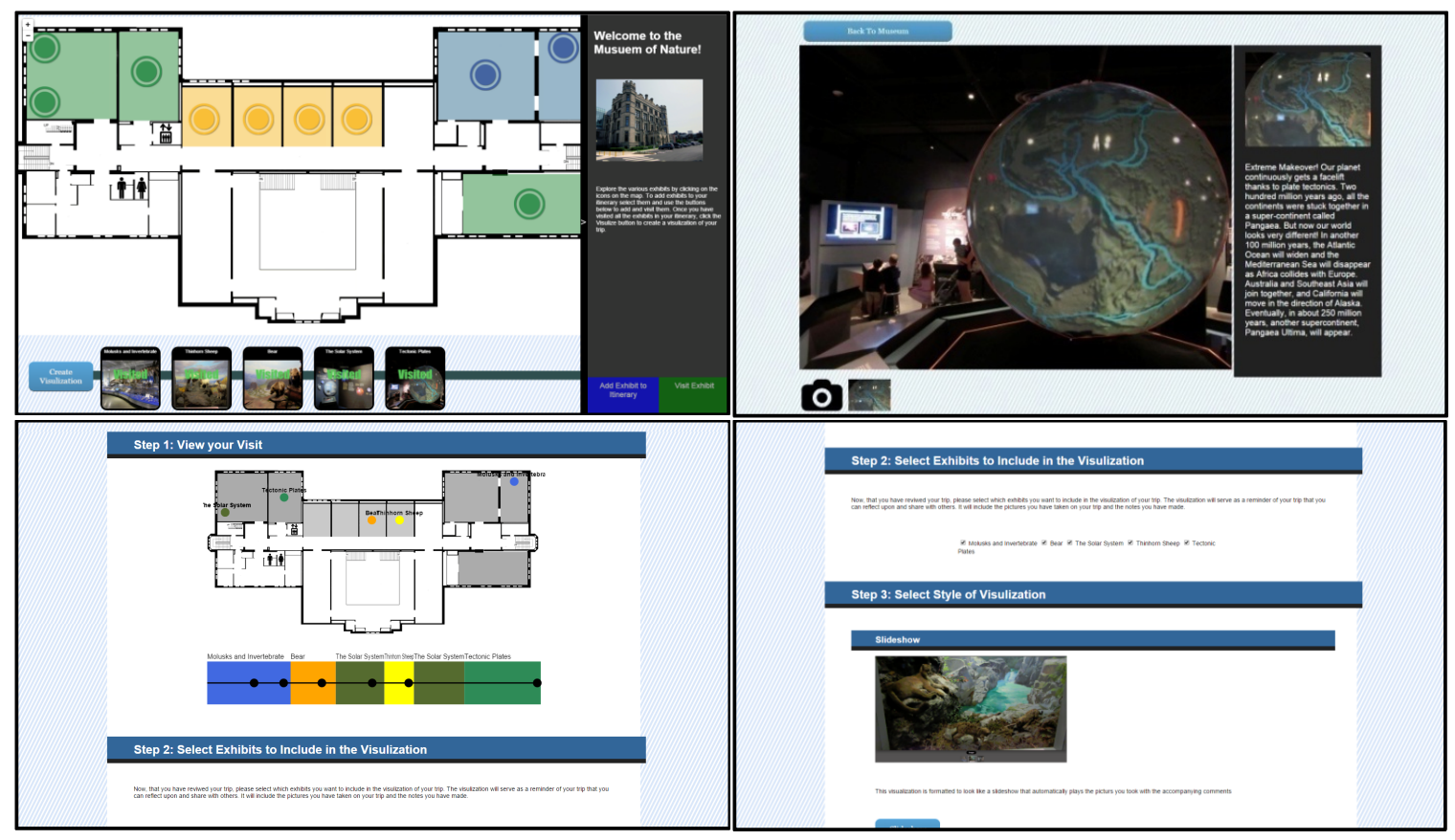

Figure 19. Stage 3 of Development

Stage 3 marked the completion of all the functionality of the prototype. It largely looked and worked as it did at the time of the tests. One of the major changes from the previous stage was the reduction of the amount of exhibits a user could visit. Implementing all the exhibits would have made the scope of the project too large. In addition, for testing 
purposes, not all the exhibits were needed as it was supposed to be a prototype and not a final version.

The UI and general style was also greatly improved. Heuristic testing was also conducted at this stage with expert users. The heuristic evaluation form can be seen in Appendix F: Heuristic Evaluation Form. The point of the heuristic testing was to ensure the prototype was as bug free as possible and easy to use.

This heuristic testing would prepare the system for final testing with users. It would allow them to evaluate the system and its ideas, rather than getting caught up with problems relating to prototyping bugs or poor system performance. 


\section{Chapter 5: User Study}

\subsection{Two Sub-Tests}

As mentioned in the introduction the two specific research questions are:

Question 1: Would each of the four narrative visualizations succeed at conveying its content in a satisfactory manner to the users?

Question 2: Would the users prefer the 'new' narrative visualizations (categorical, dramatic, sequential) to the slideshow and traditional method?

When it came time to define the research approach it became apparent that the usability test would have to be split into two separate sub-tests. Each sub-test would seek to evaluate one of the two design scenarios that formed the basis of prototype functionality. This was done because it would be impossible to accurately test the prototype in a single test because the two design scenarios had different users.

In the first scenario, the user is someone who has visited or is visiting a museum. They are going through all the different exhibits and gathering data about their trip and constructing a visual narrative out of this data. As a result they are intimately familiar with the content of the visualization as they are the ones who experienced it firsthand.

In the second scenario, the user is someone else, perhaps a family member or a friend, or someone who wants to visit the museum. Regardless, they are viewing the visual narrative of another person, a visualization they did not create. They are not intimately familiar with the experiences that happened in the museum or the content of the visualization. 
Because one user group knows about the content of the narrative visualization firsthand, and the other doesn't, two sub-tests were needed. One in which the user constructs and chooses a visual narrative and one in which the user views a narrative made by another. This separation allows for a better breakdown of some of the goals like communication. It would be very hard to test how well the narrative visualization communicates what was experienced in the museum if the user had already experienced it firsthand.

\subsection{Evaluation Criteria}

For this research project, the experiment largely involves usability testing on the prototype. It was developed in accordance of the system goals and to answer the research questions detailed in the Introduction. While the functionality of the prototype revolves around the four system goals of constructing, reflecting, communication, and sharing, these goals are abstract desired results and need to be broken down into clearer evaluation criteria. Once the criteria of a goal have been defined, it becomes much easier to determine how that goal will be measured or quantified [40]. This allows the success of the system in meeting its goals to be measured in a way that is valid and accurate [40].

In selecting usability criteria several classifications and systems of criteria were surveyed. They offered a wide variety of criteria sometimes overlapping and sometimes ambiguous. They include Nielsen's learnability, efficiency, memorability, errors, and satisfaction [40], Preece and colleagues classifications of learnability, throughput, flexibility, and attitude [39], Dix's et al. classifications of learnability, flexibility and robustness [41], and the ISO 9241-11 Standard of effectiveness, efficiency, and satisfaction [42]. 
Initially the ISO 9241-11Standard was selected as the classification to use to breakdown the goals into usability criteria. However, upon reflection, it was deemed that it would have to be appended. Usability testing seeks to go beyond the evaluation of system functionality to try to measure and assess the user's experience of the interaction and its impact upon them [41]. In order to accurately break down the desired system goals and measure the usability of specific functions of the system, more specific criteria would be needed then those provided by the ISO 9241-11 Standard of effectiveness, efficiency, and satisfaction.

Additionally, because the test is comprised of two sub-tests based on two different user scenarios, the evaluation criteria would have to be slightly different for each scenario as each scenario meets different system goals. However for both tests ISO standard 9241-11 would serve as core usability criteria. This resulted in effectiveness, operability, and satisfaction and common criteria, and flexibility and sociability as additional specific criteria in each sub-test. While these criteria together provide answer to the second research questions (superiority of narrative visualization), the flexibility and effectiveness criteria will specifically deal with the first question (success of themed visualizations in their intended themes)

\subsubsection{Operability}

Upon reflection on the context of the test and the definition of Efficiency as defined by the ISO 9241-11 usability standard, it was deemed that efficiency would be better replaced by an Operability criterion. While these two criteria are similar, efficiency is not

really a core goal of the system for the user who is visiting a museum. The web application is not very taxing computationally and the only real resource that is expended 
when using it is time. Time spent can be highly variable depending on how many exhibits a user visits. To some end, the user is even encouraged by the system to take their time when exploring the museum and building a visualization. The purpose of the system is much more based around learning and reflection than efficiency of task completion.

To this end, Operability was selected in place of efficiency to measure the usability of the system. Operability is defined as the amount of effort necessary to operate and control a software product by the user [43]. Due to the system goals, the user is more concerned with the amount of effort it takes to complete the tasks and the level of frustration they cause, rather than raw efficiency and time.

\subsubsection{Flexibility and Sociability}

In addition to the core criteria for each scenario, there is an additional usability criterion. This additional criterion was selected because it tests specific functionality unique to the sub-test.

For the first sub-test, flexibility was selected. Flexibility is defined as whether the user interface of the software product can be tailored to suit users' personal preferences [43]. It was deemed important because the first sub-test is concerned with a user constructing and choosing a visual narrative. Allowing them flexible and meaningful choice in the matter was an important part of the construction process.

For the second test, sociability was selected. It wasn't selected from any existing usability criteria or material and was selected due to the unique nature of the second scenario. Unlike traditional software, one of the goals of the system is to make it easy for the user to share what they have created and have others share with them. This goal is part of the 
larger goal of trying to create a social atmosphere and a sense of community that is present in the MEseum study that this research project used as a starting point. To this end sociability was added as a usability criteria and is defined as how well the system facilitates, engages, and prompts the user to social activity.

\subsection{Experiment Design}

\subsubsection{Method Selection}

The main method of usability testing that was selected was evaluation through user participation [41] where the users are be given a set of tasks to perform under observation in a laboratory setting [41].

This method of evaluation was selected in part because the prototype had already gone

through some heuristic testing (see Chapter 4). The design had been refined as a result of this testing and the prototype had progressed to the state where the functionality was deemed error free enough to warrant actual user testing.

\subsubsection{Qualitative and Quantitative Metrics}

The test used both qualitative and quantitative instruments of evaluation selected based upon two main factors.

The first factor was the nature of the system goals of the project. They were more qualitative in nature, and so a more qualitative means of testing was used in order to see if they had been met. Questionnaires and interviews are often seen as indirect methods because they don't measure how a user uses a system in real time. They only ask for user 
opinion on the system after use. While they are poor tools to measure some usability criteria like efficiency or number of errors, for subjective criteria relating to how the user felt about the system or how satisfied they were when using it, a survey is a direct method; many aspects of usability can be best studied by simply asking the users. This is especially true for issues relating to users subjective satisfaction [40]. The second factor in using qualitative instruments was that the testing was to be performed on a prototype. A prototype is not a final rendition of the actual system and so seeks to solicit feedback on functionality [39], rather than measure system performance or interface.

The test also aimed to be formative rather than summative [40]. That is, it was done in order to help improve the interface as part of an interactive design process. The main goal was to learn what functionality is good or bad and how the design can be improved. In contrast a summative evaluation aims at assessing overall quality of an interface for example, deciding between two alternatives or competing systems.

It was for these reasons that a more qualitative approach was chosen.

\subsubsection{Independent and Dependent Variables and Within-Subject}

\section{Testing}

The main independent variable that was manipulated was the narrative visualizations. In each test, the exhibits that the test participant visited were the same and the content served as the dependent variable. What changed was how that content was arranged into a narrative and displayed in a visual manner. The objective of the tests was to measure the usability of each visualization based upon the selected criteria and see if the hypotheses were supported. 
For the first sub-test, the participants were asked to collect some specific data and then choose one of four visual narratives. This test ascertained how well the construction process went and which visual narrative the participant felt best reflected their experience in different aspects.

For the second sub-test, the participants were asked to perform a set of tasks related to viewing narrative visualizations of exhibits they have never seen. Then they were tasked with searching for some information related to the exhibits in each of the visualizations in addition to searching for some information using the 'traditional' method of a bunch of photos in a folder. For a full breakdown of both tests and the tasks involved please see Appendix B: Test Procedure.

The experimental method that was used was within-subject, where each participant had to complete each sub-test. The order of the sub-tests was alternated where every odd numbered participant would perform the first sub-test first, and the second one second, and vice versa for every even numbered participant. This was done to reduce transfer of learning effects. Even though the second sub-test used content that the participant had never seen before, it would still be using the same narrative visualizations to present the content.

\subsubsection{Sample Population}

There was one main consideration for the sample population. This led to purposive / nonrandom sampling. It was the technological proficiency of the participants. Participants were expected to be proficient with information access, evaluation, processing and application. They were at least supposed to be able to effectively navigate the World 
Wide Web and should have had some prior experience with either web or desktop applications [8]. While they didn't have to be experts at these technologies (and the test results included a range of user proficiency in this regard) they had to at least have used such technologies before and be familiar with them.

This was a consideration because the users were testing a prototype not a final version where the test sought to ascertain how and what the users thought about the narrative visualizations and how well they preformed and were preferred, not the specific technology that they were implemented in.

\subsection{Instruments}

There are two main instruments used to evaluate the participants during testing. They are a questionnaire and timed task. The instruments are explained below. To see exactly what questions are being asked or what tasks are being performed, please refer to Appendices $B, C$, and $D$.

\subsubsection{Questionnaire}

There are two questionnaires involved in the test, one administered after each sub-test. Overall the questionnaires contained four types of questions; general, open-ended, scalar, and ranked.

General: These are some basic questions about the users' age, profession, gender, and level of computer literacy. They are used to get an idea of the demographics of the test population. 
Open-Ended: These questions are qualitative in nature and allow the user to answer at length and provide space for the user to write down their comments or thoughts free form. There are only 4 open-ended questions. The rest of the questions in the survey are closed to reduce the burden of effort on the user and encourage a high response rate.

Scalar: These questions are quantitative in nature and ask the user to judge a specific statement on a numeric scale, corresponding to a general measure of agreement or disagreement. They were measured using a seven point Likert scale. A seven point Likert scale was chosen to be able to give the users a neutral option and because it was felt a five point scale or lower would be too coarse and a 9 point scale or greater to fine.

Ranked: These questions are quantitative in nature and ask the user to rank the visualizations or visualizations and the traditional method, based upon certain criteria.

\subsubsection{Timed Task}

The user also had to complete a timed task. The task took place in the second sub-test where the user viewed the four different visualizations and the traditional method. They were required to find bits of information in the narrative visualizations and the traditional method. For details of what exactly the user had to find and the tasks they were given see Appendix B: Test Procedure.

\subsubsection{Pilot Tests}

Pilot testing was conducted on two different users to ensure the language on the questionnaires wasn't confusing and that the tests ran smoothly. The pilot test participants were not part of the actual study and the pilot tests were conducted in informal manner. The questionnaires and test procedure were revised accordingly to their comments and 
feedback. The final versions of the questionnaires and other test related material can be found in Appendices B, C and D.

\subsection{Test Breakdown}

\subsubsection{Sub-Test 1: Construction}

The first test dealt with a user scenario revolving around a user visiting a museum. The two main system goals of this scenario are Construction and Reflection. This first subtest embodies these two goals because the user is tasked with constructing a narrative visualization and has to reflect upon their experiences in the museum in order to determine which narrative visualization they prefer or which one would best embody their trip.

User Scenario: Amanda decides to visit the Museum of Nature with her family. She goes on a Saturday and takes her children through all the exhibits in the Mammals section of the museum. They take photos with their cameras and her children interact with a couple of the interactive exhibits that have simple buttons to press and knobs to turn. They also play with the interactive touch screen quizzes and spend a long time looking at and talking about the life sized animals in various dioramas. As she explores the museum, the museum app on her smartphone tags pictures that she takes, saves audio and video that she records, notes the exhibits she visits, and helps prompt and guide her along her itinerary. At the end of the day when she gets home she can use it to choose from several 
visualizations that will offer an interactive presentation of her experience that day.

Table 1. Sub-Test 1 Usability Criteria

\begin{tabular}{|l|l|}
\hline Usability Criterion & Metric \\
\hline $\begin{array}{l}\text { Effectiveness: The accuracy and completeness } \\
\text { with which specified users can achieve } \\
\text { specified goals in particular environments [41]. }\end{array}$ & 3 scalar questions using a 7-point Likert scale. \\
\hline $\begin{array}{l}\text { Operability: The amount and effort necessary } \\
\text { to control and operate a software product [43]. }\end{array}$ & 3 scalar questions using a 7-point Likert scale. \\
\hline $\begin{array}{l}\text { Satisfaction: the comfort and acceptability of } \\
\text { the work system to its users and other people } \\
\text { affected by its use [41]. }\end{array}$ & 3 questions asking user to rank visualizations. \\
\hline $\begin{array}{l}\text { Flexibility: whether the user interface of the } \\
\text { software product can be tailored to suit users' } \\
\text { personal preferences [43]. }\end{array}$ & 3 scalar questions using a 7-point Likert scale. \\
\hline
\end{tabular}

\subsubsection{Sub-Test 2: Viewing / Presentation}

The second test was based off of a user scenario that revolves around a user sharing their Visual Narrative with others. In this scenario the main user is looking at visualizations of another full of things they haven't experienced. The two main goals that this user scenario has in mind are Sharing and Communication. This second sub-test embodies these two goals because the user is having the narrative visualization of another shared with them 
and the test seeks to evaluate how well the narrative visualization communicates its content and facilitates sharing the experiences of another.

User Scenario: Reginald wants to visit the Museum of Nature while on vacation. He doesn't know much about it and goes to its website and looks at a map of its floor plan. This gives him an idea of what's inside and where everything is but not a great idea of what the experience is like. He sees that he is able to browse the narrative visualizations of others. He decides to view several to get a better idea of what the exhibits are like to actually see in person and the different things people saw. He sees that he can plan a tour using a web application on the website and then follow it with an app on his smartphone. The app would also allow him to create a visualization of his own. A few weeks later when he returns home his family wants to hear about his trip. He shows them the narrative visualization that he made and walks them through his trip. 
Table 2. Sub-Test 2 Usability Criteria

\begin{tabular}{|l|l|}
\hline Usability Criterion & Metric \\
Eith which specified users can achieve & \\
specified goals in particular environments [41]. & \\
\hline $\begin{array}{l}\text { Operability: The amount and effort necessary } \\
\text { to control and operate a software product [43]. }\end{array}$ & 5 timed tasks. \\
\hline $\begin{array}{l}\text { Satisfaction: the comfort and acceptability of } \\
\text { the work system to its users and other people }\end{array}$ & 3 questions asking user to rank visualizations. \\
affected by its use [41]. & \\
\hline Sociability: how well the system facilitates, & 3 scalar questions using a 7-point Likert scale. \\
engages, and prompts social activity. &
\end{tabular}




\section{Chapter 6: Experimental Results}

\subsection{Demographic Observation}

The user tests were conducted over two weekends. They took place at Carleton University in the HCI graduate student office. The setup was a cubicle with a desktop computer that they would use to test the prototype that had been developed. The computer was the same one that had been used for the pilot tests. The average time it took to complete the user test was approximately half an hour.

Twenty participants were tested in total. There were 8 females and 12 males and they ranged in age from 20 to 61 with varying degrees of computer literacy that they were asked to rate themselves on.

Table 3. Test Demographics

\begin{tabular}{llll}
\hline $\begin{array}{l}\text { Participant } \\
\text { Number }\end{array}$ & Gender & Age & $\begin{array}{l}\text { Computer } \\
\text { Literacy }\end{array}$ \\
\hline 1 & Female & 26 & 6 \\
2 & Male & 20 & 7 \\
3 & Male & 61 & 5 \\
4 & Female & 25 & 7 \\
5 & Male & 24 & 6 \\
6 & Female & 26 & 7 \\
7 & Female & 26 & 5 \\
8 & Male & 22 & 7 \\
9 & Male & 22 & 5 \\
10 & Male & 21 & 7 \\
11 & Female & 21 & 6 \\
12 & Male & 21 & 7 \\
13 & Male & 32 & 6 \\
14 & Female & 41 & 6 \\
15 & Male & 24 & 7 \\
16 & Male & 24 & 3 \\
17 & Male & 22 & 6 \\
18 & Female & 27 & 7 \\
19 & Male & 24 & 7 \\
20 & Female & 57 & 4 \\
\hline Average & $\mathbf{1 2 m / 8 f}$ & $\mathbf{2 8 . 3}$ & $\mathbf{6 . 0 5}$ \\
\hline & & & \\
\hline
\end{tabular}


The computer literacy on average was 6.05 out of 7 so users had a high degree of selfreported computer literacy. This combined with the consideration of selecting participants who were familiar with web technology ensured that the participants already knew how to use the technology and only had to focus on evaluating the system and the visual narratives rather than learning how to use the technology. A more finalized version of the prototype could take these things into consideration and seek to test users who aren't familiar with web technologies or who lack computer literacy. 


\subsection{Sub-Test 1: Construction}

Table 4. Sub-Test 1 Results

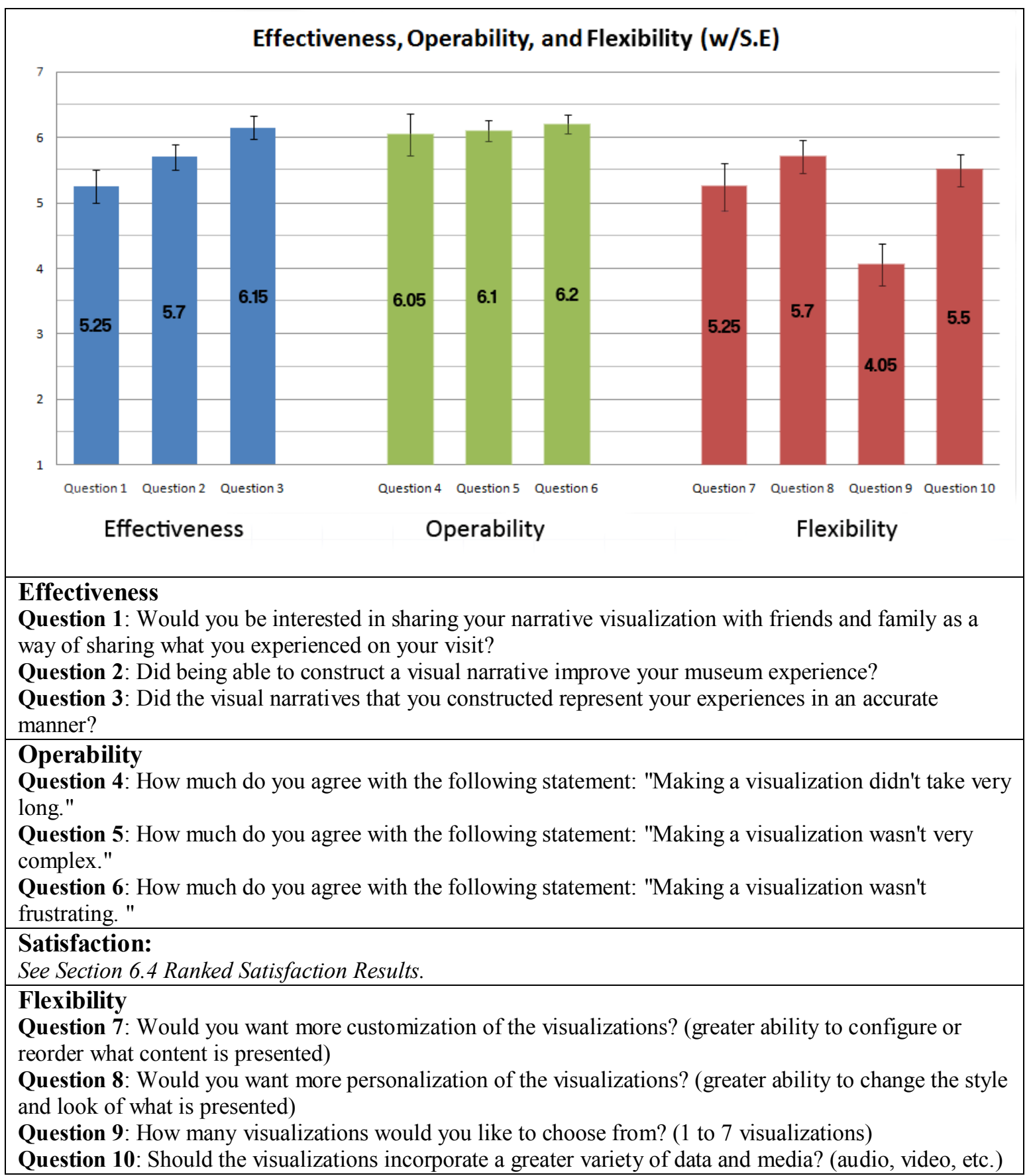




\section{Effectiveness}

The three questions dealing with effectiveness sought to ascertain how effective the process was for the users to construct a visual narrative of their experiences was in regards to core functionality and system goals. The questions asked the user to rate a dichotomy and give a rating on a 7-point Likert scale. Overall the participants answered these three questions with high ratings with an average of 5.7. The average standard error was 0.21 or about one fifth of a point on the Likert scale. These results would indicate that the users seemed to think that the construction process of the visual narratives and the visual narratives themselves were effective tools.

\section{Operability}

The three questions dealing with operability sought to ascertain how operable and easy the construction process was for the user. The questions asked the user to rate a dichotomy and give a rating on a 7 point Likert scale. Overall the users answered these questions with very high ratings with an average of 6.12 . The average standard error was 0.21 or less for all of them. Question 4 had a greater error range than the others where users seemed to be of mixed opinion that the construction process took too long. These results would indicate that the users seemed to think that the construction process of the visual narratives was not a frustrating experience and within their skill and effort.

\section{Satisfaction}

For the satisfaction results please see Section 6.4 Ranked Satisfaction Results. 


\section{Flexibility}

The four questions dealing with flexibility sought to ascertain how flexible the system was in regards to the customization and personalization of the visualizations. The average rating was 5.12 with an average standard error of 0.29 . Questions 7, 8, and 10 were rated above 4 on average and so garnered a positive response. This seems to indicate participants want greater control over the style and creation of the visualizations. Question 9 asked users to choose, from 1 to 7 , how many visualizations they would like to choose from. With the average being 4it seems that overall four visualizations was sufficient for the average participant but one more or less would also be acceptable. Question 10.b (not shown in above table) was open ended and asked participants to list what additional media they would like to be included. The following is a summary:

Table 5. Desired Additional Media

\begin{tabular}{ll}
\hline Data Type & Amount of Suggestions \\
\hline $\begin{array}{l}\text { Audio of Exhibit (narrated information, sound effects, } \\
\text { etc.) }\end{array}$ & 9 \\
Voice Notes/Journal & 1 \\
\hline Video & 10 \\
Share on Social Media & 1 \\
Interactive Animation & 3 \\
\hline
\end{tabular}




\subsection{Sub-Test 2: Viewing / Presentation}

Table 6. Sub-Test 2 Results

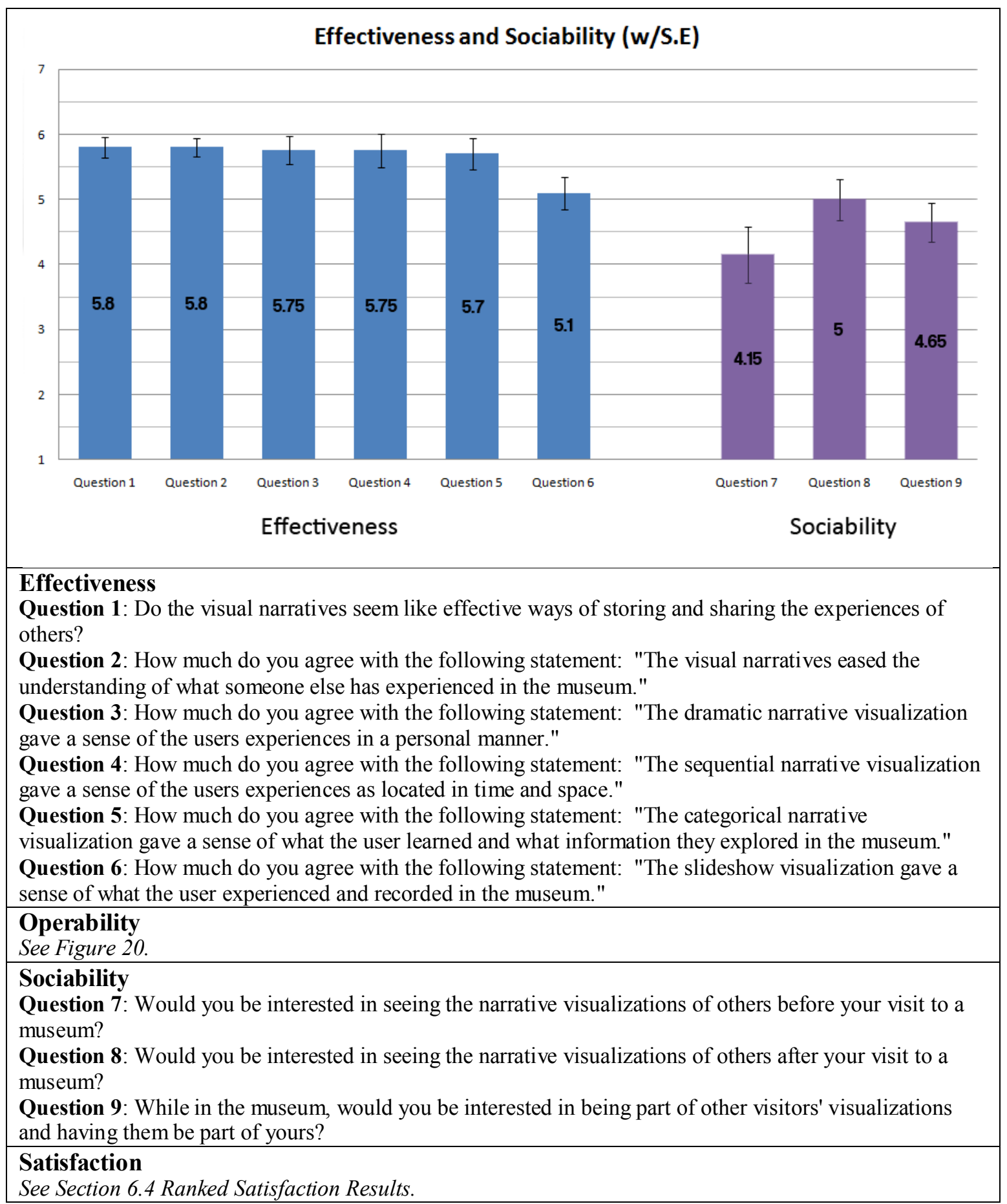




\section{Effectiveness:}

The six questions dealing with effectiveness sought to ascertain how effective the visual narratives communicate the experiences of a person's museum visit to someone who hasn't visited the museum firsthand. The questions asked the user to rate a dichotomy and give a rating on a 7-point Likert scale. These questions can be divided into two main groups.

Questions 1 and 2 deal with how effectively the narrative visualizations communicated the experiences of another's museum visit and eased the understanding of it. Participants responded positively to these questions with an average rating of 5.8 for both and an average of 0.14 standard error.

The rest of the Questions, 3 to 6, deal with how effective each narrative visualization was at what it had been designed to communicate. It did this by stating the main theme and purpose of each visualization and asking the user to rate how much they agree with it. Overall the narrative visualizations were rated with an average of 5.57 S.E. 0.24 in terms of how well they matched their indented theme and design. Participants rated the dramatic, sequential, and categorical visualizations almost identically and gave them all favourable ratings. The Slideshow visualization had a slightly less favourable rating.

\section{Operability:}

For Operability in this second test, the participant was required to complete a timed task. The reasoning to not include any ranking or rating questions for this criterion, unlike the previous sub-test, was that this test dealt solely with the viewing of narrative visualizations. As a result there wasn't a lot of complex interaction like the construction 
process in the previous test. Interaction in this second test was pretty trivial and found too easy in the pilot test to really gather meaningful results in regards to how easy or difficult it was.

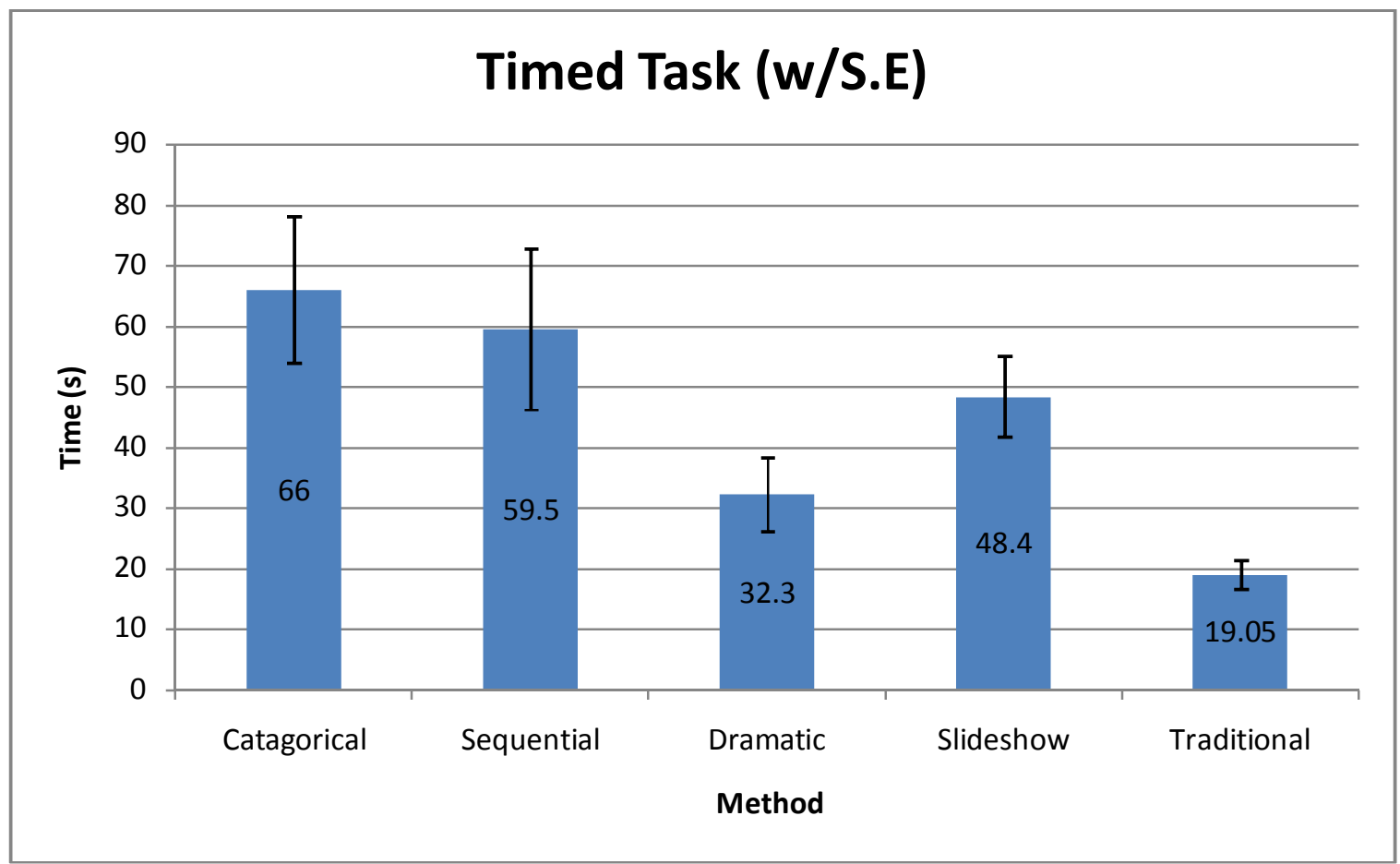

Figure 20. Results of the Timed Task

Instead, the user was tasked with answering two simple questions related to information in the narrative visualizations. Once they had identified the information and answered each question the time it took was recorded in seconds. They completed this task for each narrative visualization plus the traditional method of a bunch of pictures in a folder. The average time varies highly depending on each method and the standard error for each is high.

Overall the average time it took participants to complete the tasks was 45.05 seconds with an average standard error of 8.1 seconds. As can be seen on the graph the standard 
error for the various methods is high which causes some of them to overlap and makes it hard to draw conclusive results.

However, some observations can be made based upon how the participants completed each task and the average time it took them. Overall the traditional method preformed the best. This is likely due to built-in accelerators that the operating system provided and which several participants were observed to use, such as being able to display all individual files in a large gird, and being able to quickly scroll through them via the scrollbars. The narrative visualizations lacked such complex accelerators.

On average the categorical and sequential narrative visualizations seem to be comparable to each other and the dramatic performing better than the slideshow. Overall it seems the amount of time it took for each user to complete each task on each narrative visualization greatly varied. One reason for this that was observed was that some users were more unconcerned about the time and would leisurely read through the entire narrative visualization while others would scan and scroll and seek to find the information as quickly as possible. The difference in behaviour is probably partially a result of the intended design where the narrative visualizations were designed to encourage reflection and learning. Additionally with the mixed performance results there seems to be no strong or direct correlation between the performance of a narrative visualization and its overall rating i.e. the traditional method preformed the best but was ranked the worst and the dramatic preformed the second best and was ranked the best.

\section{Satisfaction:}

For the satisfaction results please see Section 6.4 Ranked Satisfaction Results. 


\section{Sociability:}

The usability criterion of sociability was defined as how well the system facilitates, engages, and prompts social activity. There were three questions asked. The questions asked the user to rate a dichotomy and give a rating on a 7-point Likert scale. They gave neutral responses towards the idea of seeing other visualizations before they visited a

museum, slightly positive results towards the idea of seeing the narrative visualizations of others after their visit, and a neutral response towards being part of others visualizations while in the museum. Overall the average was 4.6 with 0.35 S.E. In this regard participants didn't seem as enthusiastic towards the sociability of the system as other criteria.

\subsection{Ranked Satisfaction Results}

The satisfaction questions dealt with how comfortable and acceptable the system was to use. They focused more on whether the system was acceptable for qualitative core user goals than system functionality, as the operability questions dealt with system functionality and user frustration.

Unlike the other criteria, for the satisfaction questions users were required to rank the narrative visualizations from 1 to 4 with one being the highest. The narrative visualizations were ranked instead of rated because their content and basic functionality were the same and likely to garner very close to identical ratings. This issue arose in the pilot tests and initial feedback on narrative visualization designs. To avoid this user was asked to rank them to get a better sense of preference. 
Additionally the following 'final' rankings were determined by a combination of three main variables. This is because for some questions there would be would be a clear consensus as to what narrative visualization ranked what, but for others there would be no clear consensus. The three variables used for the final rankings were; mode ranking, the average ranking, and an assessment of the proportionality of participants that gave it a specific rank versus other ranks. For a full detailed analysis of the rankings see Appendix G: In-Depth Analysis of Satisfaction Results.

The first sub-test dealt with a user visiting a museum and constructing a narrative visualization and so the aspects it was asking the user to rank based upon satisfaction were related to core system goals of construction and reflection.

Table 7. Sub-Test 1 Satisfaction Results

\begin{tabular}{|l|l|l|l|l|}
\hline Question & $\mathbf{1}^{\text {st }}$ & 2nd & 3rd & 4th \\
\hline Reflection & Dramatic & Sequential & Categorical & Slideshow \\
& Mode: 1 or 2 & Mode: 1 or 4 & Mode: 3 & Mode: 4 \\
& Avg: 1.9 & Avg: 2.45 & Avg: 2.6 & Avg: 3.05 \\
& +-0.97 SD. & $+/-1.23$ SD & $+/-0.88$ SD & $+-1.15 S D$ \\
& +-0.22 S.E & $+/-0.23$ S.E. & $+/-0.2$ S.E. & $+/-0.26$ S.E. \\
\hline Uniqueness & Sequential & Dramatic & Categorical & Slideshow \\
& Mode: 1 & Mode: 2 & Mode: 3 & Mode: 4 \\
& Avg: 2 & Avg: 2.15 & Avg: 2.7 & Avg: 3.35 \\
& $+/-1.21$ SD. & $+/-0.93$ SD & $+/-0.98$ SD & $+/-0.93$ SD \\
& $+/-0.28$ S.E. & $+/-0.20$ S.E. & $+/-0.21$ S.E. & $+/-0.2$ S.E. \\
\hline Satisfaction & Dramatic & Categorical & Sequential & Slideshow \\
& Mode: 1 & Mode: 3 & Mode: 3 & Mode: 4 \\
& Avg: 1.75 & Avg: 2.3 & Avg:2.6 & Avg: 3.35 \\
& $+/-0.97$ SD. & $+/-1.03$ SD & $+/-1.09 S D$ & $+/-0.81$ SD \\
& $+/-0.22$ S.E. & $+/-0.23$ S.E. & $+/-0.24$ S.E. & $+/-0.18$ S.E. \\
\hline
\end{tabular}

The first question dealt with the user goal of reflection. A major component of the visual narratives was to let users reflect upon what they experienced in the museum. If the user 
didn't feel like a specific narrative visualization caused them to be all that reflective or served as a record of reflection they would be unlikely to find it satisfactory.

The second question addressed the uniqueness of the narrative visualizations. It referred to how the visual narratives were conceived by the user as unique ways of presenting their experiences.

The third question was a little more direct. It simply asked the user to rank the narrative visualizations based upon satisfaction. It offered no real specific criteria like the other two questions and simply sought to let the users rank them by holistic satisfaction.

Table 8. Sub-Test 2 Satisfaction Results

\begin{tabular}{|l|l|l|l|l|l|}
\hline Question & $\mathbf{1}^{\text {st }}$ & 2nd & $\mathbf{3}^{\text {rd }}$ & $\mathbf{4}^{\text {th }}$ & 5th \\
\hline Engagement & Dramatic & Categorical & Sequential & Slideshow & Traditional \\
& Mode: 1 & Mode: 2 & Mode: 4 & Mode: 4 & Mode: 5 \\
& Avg: 1.95 & Avg: 2.2 & Avg: 3.05 & Avg: 3.2 & Avg: 4.6 \\
& $+/-0.99$ SD & $+/-0.95 S D$ & $+/-1.4 S D$ & $+/-1.1$ SD & +-0.82 SD \\
& $+/-0.22$ S.E. & $+/-0.21$ S.E. & $+/-0.33$ S.E. & $+/-0.25$ S.E. & $+/-0.18$ S.E. \\
\hline Learning & Categorical & Dramatic & Sequential & Slideshow & Traditional \\
& Mode: 1 & Mode: 2 & Mode: 3 & Mode: 4 & Mode: 5 \\
& 1.35 & Avg: 1.9 & Avg: 3.35 & Avg: 3.7 & Avg: 4.7 \\
& $+/-0.58 S D$ & $+/-0.71$ SD & $+/-0.99 S D$ & $+/-0.66 S D$ & $+/-0.66 S D$ \\
& $+/-0.13$ S.E. & $+/-0.16$ S.E. & $+/-0.22$ S.E. & $+/-0.14$ S.E. & $+/-0.14$ S.E. \\
\hline Clarity & Dramatic & Categorical & Sequential & Slideshow & Traditional \\
& Mode: 1 & Mode: 2 & Mode: 3 & Mode: 4 & Mode: 5 \\
& Avg: 1.95 & Avg: 2.2 & Avg: 3.1 & Avg: 3.15 & Avg: 4.7 \\
& $+/-1.05 S D$ & $+/-0.95 S D$ & $+/-1.55 S D$ & $+/-0.93 S D$ & $+-0.73 S D$ \\
& $+/-0.23$ S.E. & $+/-0.21$ S.E. & $+/-0.34$ S.E. & $+/-0.2$ S.E. & $+/-0.16$ S.E. \\
\hline
\end{tabular}

The second sub-test included the traditional method in its rankings. The traditional method was a bunch of pictures from the trip contained within a folder. The participants ranked each method from 1 to 5 with one being the highest. The second sub-test dealt with the second user scenario of a user viewing narrative visualizations created by 
another and so the aspects it was asking the user to rank based upon satisfaction were related to core system goals of communication and sharing.

The first question dealt with engagement. Where in the second scenario the user is having a narrative visualization shared with them. If they don't find it very engaging they aren't likely to pay attention or really gain a sense of what the other person experienced and would be unlikely to find it satisfactory.

The second question dealt with learning. Learning is a general goal of museums and if the users felt like they were not learning much from the narrative visualizations or were not satisfied with what they were learning or how the material was being presented to them then they would be unlikely to find it satisfactory.

The third question dealt with clarity. It simply asked the user to rank the narrative visualizations based upon how clear they found them. A core system goal is communication and so this question sought to gain user feedback on how clearly the narrative visualizations communicated their content. 


\section{Chapter 7: Discussion}

\subsection{Summary of Narrative Visualizations}

A summary of how each visualization preformed in the tests is presented here along with a selection of user comments and a discussion of its overall reception.

\section{Dramatic}

The dramatic visualization ranked very highly for the majority of the ranked questions and seemed to be the one most liked overall. Results indicate it is the most reflective, and overall most satisfactory. These results go well with its design which was to be personal, and to present the users experience in a manner resembling a scrapbook. Participants seemed to enjoy this narrative visualization and many commented on how they found it engaging and how it would be useful to share on social media:

"I think with options for more personalization in the presentation (the picture sizes) and maybe adding other media (voice notes) this visualization can become really interesting and engaging."

"the theme of this one works well form a social media view. It would look good on a website."

"I liked this one the best. The 'you' centered language made the visualization more engaging." 


\section{Categorical}

The categorical visualization ranked positively overall and was highest for learning. These results go well with its intended design which was to give a sense of what the user learned and the information they explored in the museum. At an average of 66 seconds, it was the visualization that took the longest in the timed tasks. However participants ranked it second for clarity and satisfaction so its performance is more likely due to the nature of its design, where it presented a significant amount of textual information, than poor functionality. Participants seemed to enjoy this narrative visualization and many commented on how they found it helped learning:

"I think this one would work best for educational purposes. It gives a good breakdown of the exhibits and how they relate."

"I think it would be the quickest way to find the info you needed under this type."

"the additional comments and information were helpful in fleshing out the ideas I already got. The extra pictures made it seem more professional as expanded on what I put there myself. This was my favourite."

\section{Sequential}

Besides being ranked the most unique, the sequential narrative visualization ranked neutral, being neither the highest nor lowest ranked visualization. However, according to the effectiveness results, users did seem to understand and agree with its intended design of giving a sense of the user's experiences as located in time and space. Overall it seems like participants thought the design was effective and unique but didn't particularly like it over the other narrative visualizations. This is likely due to two factors. The first being 
the implementation, participants commented that they had some issues with using it. It might have ranked higher if it had been tweaked a bit and undergone some more user testing.

"I liked the idea of sequential visualization although the text display was a bit difficult to read. I would prefer to see a bigger popup when I click on a picture."

"visual indicator of start location/exhibit icon would've been helpful in addition to different coloured lines at each point of the journey."

"One thing that would be preferred is a larger pop-up for visited exhibits to account for more info. Dotted line pathway at right angles to destination would look more appealing (if an option)."

"least useful, most interesting"

The second factor is that the sequential visual narrative might be better suited towards different scenarios than the ones used. It would probably be more useful in a larger museum or even an outdoor space where there are numerous things to see and a greater sense of time and space than a small museum building. It is probably the narrative visualization that is the most dependent upon where the trip is taking place in.

"Most interesting part would be to use the sequence for my own visit but otherwise not sure why I care at what time people arrived and left the exhibit."

"this one might work well for any large exhibits, museum. It would be great to use one for museums to plan your visit a second time. I like that my notes and pictures were included." 


\section{Slideshow}

The slideshow ranked last in the qualitative satisfaction aspects of Sub-Test 1. It also came second last in the qualitative satisfaction aspects of Sub-Test 2 which included the traditional method not part of Sub-Test 1. Overall, the slideshow was ranked very poorly by participants. However it came third out of five in the timed tests with an average time of 48.4 seconds and so was neutral in that regard. The participant comments were very informative regarding the difference between ranking and performance. Overall the participants found the slide show narrative visualization to be too traditional and boring to merit a high ranking over the others despite its performance.

"Makes you feel like you are in person's shows who has already visited the museum. Just cannot learn a lot about the exhibit itself."

"forced user to retain information."

"very traditional, gave sense of what a user wanted to see."

"slightly boring."

"this was boring, did not care."

\section{Traditional}

The traditional method really wasn't a narrative visualization method. It was quite simply the common method of storing pictures and information about a trip wherein all the individual pictures and files are placed together in a folder on a computer. It was included in the second test so the results of looking through the information could be compared with the visualizations which seek to present the information in a different format. 
Unsurprisingly, it was ranked last in all aspects by participants by a wide margin (75$80 \%$ ). However with an average time of 19.05 seconds it performed the best out of all the methods in the timed tasks. This is likely due to the fact that having all the pictures and files as separate things, instead of presented in some kind of holistic manner, made it very easy to use accelerators in the windows operating system (such as metadata and indexing) to sort through the data. However participants didn't seem to enjoy this method as much when it came to viewing a presentation of somebody's else's experiences and so ranked it poorly.

\subsection{Discussion}

The first sub-test dealt with visiting a virtual museum, viewing exhibits and gathering data, and constructing a visual narrative out of that data. The participants rated the prototype overall highly in both effectiveness (avg: 5.7+/- 0.21 S.E) and operability (avg: $6.12+/-0.21$ S.E). The second sub-test dealt with viewing the visual narratives created by another person detailing their museum visit. The participants also rated the prototype overall high in both effectiveness (avg: $5.65+/-0.21$ S.E).In terms of operability the average time, in seconds, it took for the timed task was low $(45.05+/-8.1$ S.E) given the nature of the task.

The results from both tests indicate that the participants understood how to construct a visual narrative and the purposes of constructing one. They thought they were effective and accurate tools for sharing one's experiences in a museum and that they improved the museum experience. 
These operability results from both tests seem to indicate that users did not find the simulated museum visit, and the construction process of making a narrative visualization, to be too frustrating, complex, or lengthy. The prototype carried out its intended functions in an easy-to-use manner that did not distract from the higher level concepts and designs that were being tested or influence them in a negative fashion.

These results provide a positive answer to the first research question that each narrative visualization would succeed at its theme. Participants responded positively to the effectiveness and operability of each theme of the narrative visualizations. While they definitely ranked some visualizations as more preferable to others, they thought that overall the visualizations preformed well and were implemented in a way that matched their design. They responded positively to statements regarding a summarized design concept of each narrative visualization matching its design and implementation.

The flexibility results also support the positive answer to the first research question. Participant response was mixed but overall positive where they responded positively to having different narrative visualization designs to choose from and that having multiple narrative visualizations was beneficiary. They also indicated that they wanted the narrative visualizations to incorporate a larger number of data types (ranging from audio, to video, to social media data). However, for the average user, 4 visualizations to choose from was enough but they would like to have more control over the style and creation of the narrative visualizations that they are given.

Lastly, the user comments seem to also support the success of themed visualization in their purposes. Many users remarked on how they liked the themed nature of a narrative 
visualizations and what they found interesting about them. They seemed to find value in having each narrative visualization focused on arranging and presenting the information with a different theme. Many even commented they could see how a certain narrative visualization would work really well in a specific context, such as the Dramatic one being working well for sharing over social media.

However, there is still issue of preference and ranking. The narrative visualizations that the participants responded to least positively, was the slideshow in the first test. In the second test, the traditional method was least preferred with the slideshow the second least preferred. The results of the timed task are of mixed results in terms of how the 'new' narrative visualizations compared to the more traditional methods with no strong or clear correlation between rating and performance. The 'new' narrative visualizations may have been rated highly despite their high or low performance due to the novelty of them. At the same time, being "new" may have had negative effect on the user evaluation due to lack of familiarity. Overall, the effect of novelty can only be properly studied through a longer term research. However, none of the participants indicated in comments that they actually thought the narrative visualizations took too long to find information nor did they rate or rank them poorly in terms of communication or clarity. It seems that the performance was not a concern to the users, at least within the timeframe that it took them to complete the task.

The difference between the response and rankings of the 'new' narrative visualizations (categorical, sequential, dramatic) and the more traditional methods (Slideshow, pictures in a folder) largely provides a positive answer to the second research question, 
confirming that participants prefer the narrative visualizations over the traditional methods.

\subsection{Limitations}

There are several limitations in this survey. The first and foremost was that usability testing was carried out on a prototype which may differ from a more finalized version. The final version could be developed as a mobile smartphone application and provide more convenient interface and tools, and could be tested in a real museum instead of a virtual one. Both these two factors may affect the results and user opinions.

Secondly, selection of participants who had used web technologies before and were computer literate meant computer literature users or those unfamiliar with web technology would probably have more usability issues with using the system, although such systems could be considered as ones targeted at more digitally experienced users.

Additionally, there were various subjective questions that would ask the user and try to get a sense of how well the system preformed in terms of its goal of causing the user to reflect upon what they had experienced. However, there was no test of this goal where users were asked a few days or weeks later after creating a narrative visualization to use the system and reflect upon their visit and to see how it aided them in remembering facts about their trip or discover new things. In this regard, the tests were subjective and measuring users' initial perception of reflective abilities in the system.

There are also a few limitations regarding the test results. The most prominent is with the ranking questions. For some questions a clear majority of the participants would give 
each narrative visualization a specific rank. But for other questions participants would give more mixed rankings and participant consensus would be unclear. Testing with additional participants would probably grant a clearer consensus.

The novelty of the 'new' narrative visualizations compared to the slideshow and traditional method may have been a factor in their rating which requires longer term studies to understand. 


\section{Chapter 8: Conclusion and Future Work}

This research sought to evaluate several different designs and implementations of visual narratives through the development of a prototype that would enable a user to visit a virtual museum, and create and share a narrative visualization of their experiences.

The main contributions of this study are:

1. Identification and design of four narrative visualization styles.

2. Design of a prototype museum system

3. Classifying Exhibits Types

4. Usability testing of four different narrative visualization schemes

The research was conducted primarily with the intent to explore the two research questions:

Question 1: Would each of the four narrative visualizations succeed at conveying its content in a satisfactory manner to the users?

Question 2: Would the users prefer the 'new' narrative visualizations (categorical, dramatic, sequential) to the slideshow and traditional method?

It sought to do this though the design of narrative visualizations which were formed after a literature review of topics including Information Visualization, Narrative in Digital Media, and Narrative Visualization.

These narrative visualizations would enable the user to construct a visual narrative out of all the data that they had collected on their museum visit. They could present their visual narrative to friends and family members and it would serve as a presentation and storage 
tool that would allow the user and others to share and reflect on what they experienced. A prototype was developed to embody these narrative visualizations and usability testing was carried out on the prototype, with a variety of metrics used to determine how well the narrative visualizations succeeded at their core goals. The evaluation criteria included effectiveness, operability, flexibility, sociability and satisfaction. The evaluation results were used to answer the research questions.

Overall, the results from the user study conducted show that narrative visualizations met their core goals of construction, sharing, communication, and reflecting. They were successful at meeting their evaluation criteria in a positive manner; they were seen as effective tools that were easy to use and operate. The first question was answered positively in this manner where each visualization preformed well with its theme understood by the participants and each seen as excelling in different contexts. Additionally users desired for there to be greater customization and data types present in the narrative visualizations.

The four individual themes that the participants could choose from where well received however some were preferred over others. The 'new' visual narratives (categorical, sequential, dramatic) were ranked higher than the more traditional methods (Slideshow, pictures in a folder). The second research question was also answered positively in this manner.

Further efforts can be considered for this project in future. The first and probably the most important one is to develop a new version that works on a mobile device and can be deployed and tested in a real museum. While, sharing and organizing data can be done on 
a PC and at home, actual visit and data collection should happen on site and requires a more finalized design to be implemented via a mobile application that is used in real museums.

Future developments should also include a greater variety of data types such as audio and video, in the narrative visualizations. The results of this study indicate that participants found narrative visualizations to be effective tools that were easy to use and that they succeeded at conveying experiences with different themes. Additional and more rigorous testing could be performed on these themes, either in conjunction with the ones presented here or as separate tests.

Last but not least, more usability tests including a wider range and larger number of participants, and also different exhibit/museum types will help identifying the advantages and disadvantages of the proposed narrative visualization. 


\section{Bibliography}

1. "The World Museum Community: Museum Definition", Internet:

http://icom.museum/the-vision/museum-definition/, [March 24th 2015]

2. E. P. Alexander and M. Alexander. Museums in Motion: An Introduction to the History and Functions of Museums. Plymouth, UK.: AltaMira Press, 2008.

3. P. F. Marty and Katherine Burton Jones. Museum Informatics: People, Information and Technology in Museums. New York, NY: Routledge, Taylor \& Francis group, 2008.

4. S. Campbell and R. Ling. "Effects of mobile communication". Internet: http://sites.google.com/site/mobilephonesandsociety/Home/week4/CampbellandLingmobilemediaeffects.pdfinhttps://blog.itu.dk/dmkse2008/files/2008/09/campbell-and-ling-mobile-media-effects.pdf. [March 3, 2015].

5. N.D. Lane. et al. "A survey of mobile phone sensing". IEEE Communications Magazine, vol. 48, no. 9, pp. 140-150, 2010.

6. S.W. Campbell and Y.J Park. "Social Implications of Mobile Telephony: The Rise of Personal Communication Society". Sociology Compass, vol. 2, no. 2, pp. 371$387,2008$.

7. Kevin Walker. "Story structures: Building narrative trails in museums". Technology-mediated narrative environments for learning. Rotterdam: Sense Publishers, 2006, pp. 103-114.

8. Efetobore Mike-Ifeta, "MEseum: Constructing Digital Narratives For The Museum Visitor," M.A thesis, HCI, Carleton University, Ottawa, Ontario, 2014.

9. J. Jae, J. M. Esteve, J. A. Mochol1, et al. "MoMo : enabling hybrid museums,' IEE Proceedings - Software, vol. 152, no. 5, pp. 245-251, 2005.

10. I. Roes, N. Stash, Y. Wang, and L. Aroyo, "A personalized walk through the museum: The CHIP interactive tour guide," Proceedings 27th SIGCHI Conference on Human Factors in Computing Systems, pp. 3317-3322, 2009.

11. S. Bosman and B. Groenendaal, “Gentleguide: An exploration of haptic output for indoors pedestrian guidance" in Human-Computer Interaction with Mobile 
Devices and Services, Luca Chittaro (Ed), Springer Berlin Heidelberg, 2003, pp. 358-362.

12. L. Naismith, M. Sharples, and J. Ting, "Evaluation of CAERUS: a context aware mobile guide," in Mobile Technology: The Future of Learning in Your Hands, mLearn 2005 Book of Abstracts, 4th World Conference on mLearning, H. V. D. Merwe \& T. Brown (Eds.), Cape Town: mLearn, 2005, pp. 50.

13. A. Angus, D. Papadogkonas, G. Papamarkos, G. Roussos, G. Lane, K. Martin, N. West, S. Thelwall, Z. Sujon, and R. Silverstone, "Urban social tapestries," IEEE Pervasive Comput., vol. 7, no. 4, pp. 44-51, Oct. 2008.

14. J. Bruner, Actual Minds, Possible Worlds. Cambridge, Massachusetts:Harvard University Press, 1986.

15. J. Bruner. Acts of meaning. Cambridge, Massachusetts: Harvard University Press, 1990.

16. J. Bruner. The culture of Education. Cambridge, Massachusetts: Harvard University Press, 1996.

17. N. Gershon and W. Page. "What storytelling can do for information visualization". Communications of the ACM, vol. 44, no. 8, pp. 31-37, Aug. 2001.

18. H. Corvellec. "Elements of narrative analysis". Göteborg University, School of Business, Economics and Law, GRI reports, nr. 2006:6, 2006, available: $<$ https://gupea.ub.gu.se/handle/2077/2980>

19. Marie-Laure Ryan. "Beyond Myth and Metaphor - The Case of Narrative in Digital Media". Game Studies: The International Journal of Computer Game Research, vol. 1, no. 1, pp 1-13, July 2001.

20. C. Bateman. Game Writing: Narrative Skills for Videogames. Charles River Media, Inc., 2006.

21. M.Cavazza, F. Charles, \& S. J. Mead. "Sex, Lies and Videogames: an Interactive Storytelling Prototype". In Proceedings of the AAAI 2002 Symposium on Artificial Intelligence and Interactive Entertainment, pp. 13-17, 2002.

22. E. EFox. "The next generation of interactive technologies". Communications of the $A C M$, vol. 32, no. 7, pp. 872-880, 1989. 
23. F. Charles, M. Lozano, S. J.Mead, et al. "Planning formalisms and authoring in interactive storytelling". In Proceedings of TIDSE, Vol. 3, March 2003.

24. G. Frasca. "Simulation versus narrative". The video game theory reader, pp. 221$235,2003$.

25. R. Kosara, and J. MacKinlay. "Storytelling: The next step for visualization". IEEE Computer (Special Issue on Cutting-Edge Research in Visualization), vol. 46, no. 5, pp. 44-50, 2013.

26. J. Gray, L. Chambers, and L. Bounegru. The data journalism handbook. " O'Reilly Media, Inc.", 2012.

27. G. Dove and S. Jones. "Narrative Visualization: Sharing Insights into Complex Data". Paper presented at the Interfaces and Human Computer Interaction (IHCI 2012), Lisbon, Portugal. Jul 21 - 23, 2012.

28. M. Tory, and M. Torsten. "Human factors in visualization research." Visualization and Computer Graphics, IEEE Transactions on 10, no. 1, pp. 72-84, 2004.

29. M. Voigt, M. Franke, K. Meißner. "Capturing and Reusing Empirical Visualization Knowledge". In UMAP Workshops, 2013.

30. G. Robertson, R. Fernandez, D. Fisher, et al. "Effectiveness of animation in trend visualization". Visualization and Computer Graphics, IEEE Transactions on, vol. 14, no. 6, pp. 1325-1332, 2008.

31. W. Willett, J. Heer, and M. Agrawala. "Scented widgets: Improving navigation cues with embedded visualizations." Visualization and Computer Graphics, IEEE Transactions on 13, no. 6, pp. 1129-1136, 2007.

32. B. Moghaddam, Q. Tian, N. Lesh, C. Shen, and T. Huang. "Visualization and user-modeling for browsing personal photo libraries." International Journal of Computer Vision 56, no. 1-2, pp. 109-130, 2004.

33. Charles Minard. "Figurative Map of the successive losses in men of the French Army in the Russian campaign 1812-1813". Internet:

$<$ http://en.wikipedia.org/wiki/File:Minard.png> [Mar. 3, 2015]. 
34. J. Hullman and N. Diakopoulos. "Visualization rhetoric: Framing effects in narrative visualization". IEEE Transactions on Visualization and Computer Graphics, $\quad$ vol. 17, no. 12, pp. 2231-2240, 2011.

35. E. Segel, J. Heer. "Narrative Visualization: Telling Stories with Data". IEEE Transactions on Visualization and Computer Graphics. vol. 16, no. 6, pp. 11391148, 2010.

36. Canadian War Museum. "Canadian War Museum". Internet: $<$ http://www.warmuseum.ca/home/> [Mar. 3, 2015].

37. Canadian Museum of Nature. " Canadian Museum of Nature". Internet: < http://nature.ca/en/home> [Mar. 3, 2015].

38. Canadian Museum of History. "Canadian Museum of History ". Internet: < http://www.historymuseum.ca/home > [Mar. 3, 2015].

39. J. Preece, Y. Rogers, H. Sharp, D. Benyon, S. Holland, and T. Carey. HumanComputer Interaction. Reading, MA: Addison-Wesley. 1994.

40. J. Nielsen. Usability Engineering, 1st ed. San Diego, USA: Academic Press, 1993.

41. A. Dix, J. Finlay, G. D. Abowd, R. Beale. Human-Computer Interaction. 3rd ed. Essex, England: Pearson Edu. Ltd. 2004.

42. D. Alonso-Ríos, A. Vázquez-García, E. Mosqueira-Rey et al. "Usability: A Critical Analysis and a Taxonomy". International Journal of Human-Computer Interaction, vol. 26, no. 1, pp. 53-74, 2009.

43. A. Seffah, M. Donyaee, R.B. Kline, and H.K. Padda. "Usability measurement and metrics: A consolidated model". Software Quality Journal, vol. 14, no. 2, pp. 159178 , June 2006. 


\section{Appendix A: Consent Form}

\section{Participant Informed Consent Form}

You have been solicited as a research participant for our project entitled:

\section{Crafting Narrative Visualizations of Museum Experiences}

The research is being conducted by:

- Dr. Ali Arya, School of Information Technology, Carleton University, Ottawa, Canada, arya@carleton.ca

- Jesse Gerroir, Human Computer-Interaction, School of Information Technology, Carleton University, Ottawa, Canada, jessegerroir@cmail.carleton.ca

\section{Purpose}

The purpose of this user test is to evaluate the extent to which the application of creating a visual narrative of a users experience in a museum using interactive technology can enhance an individual's museum visit. The functions to be evaluated at this stage of development are:

- The user's ability to independently construct a visual narrative.

- The effectiveness of the visual narrative in reflecting the users experiences.

- The ability of the visual narrative to communicate its contents and be shared.

\section{Task Description}

The user's tasks are divided into two main tests. The first test is for the user to visit several exhibits in a virtual museum and collect data from each virtual exhibit in the way of pictures and text. Then view various ways they can be visualized and a narrative of their experiences presented.

The second test is for the user to view visualizations based upon a premade trip and compare how each visualization presents the information.

\section{Dissemination}

This research is a part of a project supervised by Dr. Ali Arya (School of Information Technology) and conducted by Jesse Gerroir (MASc, Human Computer Interaction, Department of Information Technology) towards a Master's degree at Carleton University. The result of this research will be used in Jesse Gerroir's thesis and might also be published and/or presented in conferences and/or journals, as well as grant applications. 


\title{
Anonymity/Confidentiality
}

You may choose to provide your names and emails or choose to remain anonymous. If you do provide your name and email, however, they will not be used or published in the research, as they will only be kept for possible future verification.

\section{Risks}

There are no known risks associated with this activity.

\section{Right to Withdraw}

As a participant, you may withdraw at any time for any reason.

This research has been reviewed and cleared by the Carleton University Research Ethics Board (REB) and questions and concerns can be addressed to the REB chair.

\author{
Research Ethics Board: \\ Professor Andy Adler, Chair \\ Research Ethics Board \\ Carleton University Research Office \\ Carleton University \\ 1125 Colonel By Drive \\ Ottawa, Ontario K1S 5B6 \\ Tel: 613-520-2517 E-mail: ethics@carleton.ca
}

Your signature below indicates that you have read the above and voluntarily agree to participate. If you have any questions, please ask them before signing.

$* * *$ I have read and understand the above information ***

Participants Name (mandatory):

Phone Number (optional):

Email (optional):

Gender (mandatory):

Age (mandatory):

Occupation (mandatory):

Nationality (mandatory):

Signature (mandatory): Date (mandatory): 


\section{Appendix B: Test Procedure}

\section{Test Instructions}

Thank you once again for your willingness to participate in the usability study for my graduate thesis research. This usability study is divided into two main sections. A construction section where you will be constructing a visual narrative of a simulated museum visit and a viewing section where you will view previously constructed visual narratives. Each section is followed by a short survey.

\section{Sub-Test 1: Construction}

To begin please navigate to the following website in Google chrome:

http://jessegerroir.com/thesis/current/

Please press the "Reset System" link before you begin. This will reset the system to its starting conditions. You should see a popup saying that the system has been successfully rest. Then click the "Museum 1" link to proceed to the system proper.

\section{Task 1: Museum Explorer}

1. Add the Thinhorn Sheep exhibit to your itinerary.

2. Remove the Thinhorn Sheep exhibit from your itinerary.

3. Add the following exhibits to your itinerary:

- Cougar

- Marine Mammals and Birds

- Beaver

- Meteorites

- Three Types of Rock

4. Click on an exhibit in your itinerary.

5. Visit the exhibit by clicking the 'visit exhibit' button.

\section{Task 2: Exhibit Explorer}

1. Click on an artefact hotspot.

2. Take a picture of the artefact.

3. Add a note to the picture.

4. Return to the museum. 


\section{Task 3: Gathering Data}

1. Visit all the other unvisited exhibits on your itinerary.

2. Take at least 3 more pictures and add at least 3 more notes.

3. When you are done exploring all the exhibits click the Visualize button.

\section{Task 4: Visualization}

1. View your visit.

2. Select which exhibits you'd like to include in your visualization.

3. Select one of the four visualization styles (it will open in a new tab).

4. Explore the visualization to get a sense of it.

5. Go back and explore the other four visualization styles until you have explored them all.

\section{Task 5: Survey}

1. Please fill out Survey 1. 


\section{Sub-Test 2: Viewing}

To begin please navigate to the following website in Google chrome: http://jessegerroir.com/thesis/frozen/

Have the Observer setup the system for you by completing the following:

1. Click the "Museum 1" link to proceed to the system proper.

2. The itinerary should already be filled with visited exhibits. Click the 'Create Visualization' button.

3. Select all of the exhibits as ones that you'd like to include in your visualization.

\section{Timed Tasks}

You will be timed for each of these tasks. Each tasks basically involves viewing a visualization and trying to locate two items depending on visualization type.

Task 1: in the Categorical Visualization try to find:

- The number of Thinhorn sheep in the Thinhorn sheep exhibit.

- A trait that all Mollusks share.

Task 2: in the Sequential Visualization try to find:

- The number of Thinhorn sheep in the Thinhorn sheep exhibit.

- The date or time the museum was visited on.

Task 3: in the Dramatic Visualization try to find:

- The number of Thinhorn sheep in the Thinhorn sheep exhibit.

- How many exhibits were visited.

Task 4: in the Slideshow Visualization try to find:

- The number of Thinhorn sheep in the Thinhorn sheep exhibit.

- the number of pictures taken.

Task 4: in the Traditional Method try to find:

- The number of Thinhorn sheep in the Thinhorn sheep exhibit.

- the number of pictures taken.

\section{Survey}

- $\quad$ Please fill out Survey 2. 


\section{Appendix C: Survey 1 - Construction}

Participant Number:

This section should be completed after the participant has performed sub-test one. Please answer all of the following questions as best as you can. You can write on this paper.

Please rate your computer literacy:

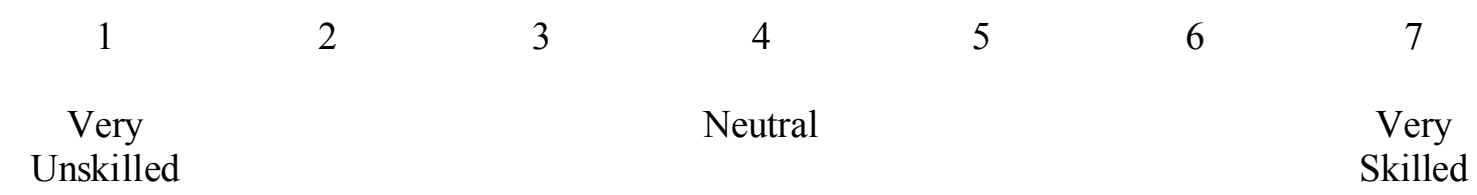

For the following questions please circle a number according to how much you agree or disagree with the question.

1. Would you be interested in sharing your narrative visualization with friends and family as a way of sharing what you experienced on your visit?

$\begin{array}{llllllll}1 & 2 & 3 & 4 & 5 & 6 & 7\end{array}$

Very Neutral Nery

uninterested. interested.

2. Did being able to construct a visual narrative improve your museum experience?

$\begin{array}{llllllll}1 & 2 & 3 & 4 & 5 & 6 & 7\end{array}$

No Neutral

improvement

Big

improvement

3. Did the visual narratives that you constructed represent your experiences in an accurate manner?

1

Very

Inaccurate
2

3

4

5

6

7

Neutral 
4. How much do you agree with the following statement: "Making a visualization didn't take very long."
1
2
3
4
5
6
7

Strongly

Neutral

Strongly

Disagree

Agree

5. How much do you agree with the following statement: "Making a visualization wasn't very complex."
1
2
3
4
5
6
7

Strongly

Neutral

Strongly

Disagree

Agree

6. How much do you agree with the following statement: "Making a visualization wasn't frustrating."

1

Strongly

Disagree
2

3

4

5

6

7

Neutral
Strongly

Agree

7. Would you want more customization of the visualizations? (greater ability to configure or reorder what content is presented)
1
2
3
4
5
6
7

Less

Neutral

More

Customization

Customization

8. Would you want more personalization of the visualizations? (greater ability to change the style and look of what is presented)

1

Less

Personalization
2 3

Neutral
45

(1)

(1)
6 7

More

Personalization

9. How many visualizations would you like to choose from?

$\begin{array}{ccccccc}1 & 2 & 3 & 4 & 5 & 6 & 7 \\ \text { One } & & \text { four } & & & \text { Seven or }\end{array}$


10(a). Should the visualizations incorporate a greater variety of data and media? (audio, video, etc.)

$\begin{array}{ccccccc}1 & 2 & 3 & 4 & 5 & 6 & 7 \\ \text { Less } & & \text { Neutral } & & & \text { More } \\ \text { Variety } & & & & \text { Variety }\end{array}$

10(b). If you answered positively towards 10a please list in order the additional media you'd like to see incorporated:

For the following questions please rank the visual narratives with a number from 1 to 4.1 being most preferred and 4 being least preferred.

11. Which visual narratives helped you reflect upon your experiences the most?

Dramatic

Sequential

Categorical

Slideshow

12. Which visual narrative conveyed your experiences the most uniquely?

Dramatic

Sequential

Categorical

Slideshow

13. Which visual narrative conveyed your experiences the most satisfactory?

Dramatic

Sequential

Categorical

Slideshow 


\section{Appendix D: Survey 2 - Viewing}

Participant Number:

This section should be completed after the participant has performed sub-test two. Please answer all of the following questions as best as you can. You can write on this paper.

For the following questions please circle a number according to how much you agree or disagree with the question.

1. Do the visual narratives seem like effective ways of storing and sharing the experiences of others?
1
2
3
4
6
7

Very

Neutral

Very

ineffective

Effective

2. How much do you agree with the following statement: "The visual narratives eased the understanding of what someone else has experienced in the museum."
1
2
3
4
5
6
7

Strongly

Neutral

Strongly

Disagree

Agree

3. How much do you agree with the following statement: "The Dramatic narrative visualization gave a sense of the users experiences in a personal manner."
1
2
3
4
5
6
7

Strongly

Neutral

Strongly

Disagree

Agree

4. How much do you agree with the following statement: "The Sequential narrative visualization gave a sense of the users experiences as located in time and space."
1
2
3
4
5
6
7

Strongly

Neutral

Strongly

Disagree

Agree 
5. How much do you agree with the following statement: "The Categorical narrative visualization gave a sense of what the user learned and what information they explored in the museum."
1
2
3
4
5
6

Strongly

Neutral

Strongly

Disagree

Agree

6. How much do you agree with the following statement: "The Slideshow visualization gave a sense of what the user experienced and recorded in the museum."
1
2
3
4
56
7

Strongly

Neutral

Strongly

Disagree

Agree

7. Would you be interested in seeing the narrative visualizations of others before your visit to a museum?

$\begin{array}{ccccccc}1 & 2 & 3 & 4 & 5 & 6 & 7 \\ \begin{array}{c}\text { Very } \\ \text { Uninterested }\end{array} & & \text { Neutral } & & & \text { Very } \\ \text { Interested }\end{array}$

8. Would you be interested in seeing the narrative visualizations of others after your visit to a museum?

1

Very

Uninterested

$2 \quad 3$

4

Neutral

9. While in the museum, would you be interested in being part of other visitors' visualizations and having them be part of yours?

1

Very

Uninterested
2

3

4

Neutral
$5 \quad 6$

7

Very Interested 
For the following questions please rank the visual narratives and the traditional method (folder full of pictures from the trip) with a number from 1 to 5 . 1 being most preferred and 5 being least preferred.

10. Which was the most engaging?

Dramatic

Sequential

Categorical

Slideshow

Traditional

11. Which helped you learn the most about the exhibits?

Dramatic

Sequential

Categorical

Slideshow

Traditional

12. Which communicated the experiences the most clearly?

Dramatic

Sequential

Categorical

Slideshow

Traditional 
Please use the space below to offer any additional comments on any of the visual narratives:

Catagorical:

Dramatic:

Sequential:

Slideshow:

Additional

Comments: 


\section{Appendix E: Observer Record}

Participant Number:

Sub-test taken first:

Sub-test taken second:

\begin{tabular}{|l|l|}
\hline Visualization & Time \\
\hline Categorical: & \\
\hline Sequential: & \\
\hline Dramatic: & \\
\hline Slideshow: & \\
\hline Traditional: & \\
\hline
\end{tabular}

Participant Number:

Sub-test taken first:

Sub-test taken second:

\begin{tabular}{|l|l|}
\hline Visualization & Time \\
\hline Categorical: & \\
\hline Sequential: & \\
\hline Dramatic: & \\
\hline Slideshow: & \\
\hline Traditional: & \\
\hline
\end{tabular}

Participant Number:

Sub-test taken first:

Sub-test taken second:

Visualization

Time

Categorical:

Sequential:

Dramatic:

Slideshow:

Traditional:

Participant Number:

Sub-test taken first:

Sub-test taken second:

\begin{tabular}{|l|l|}
\hline Visualization & Time \\
\hline Categorical: & \\
\hline Sequential: & \\
\hline Dramatic: & \\
\hline Slideshow: & \\
\hline Traditional: & \\
\hline
\end{tabular}

Participant Number:

Sub-test taken first:

Sub-test taken second:

\begin{tabular}{|l|l|}
\hline Visualization & Time \\
\hline Categorical: & \\
\hline Sequential: & \\
\hline Dramatic: & \\
\hline Slideshow: & \\
\hline Traditional: & \\
\hline
\end{tabular}




\section{Appendix F: Heuristic Evaluation Form}

\section{Name:}

\section{Date:}

For this heuristic evaluation you will be given a series of tasks. Please try to complete each task the best you can.

\section{Heuristics:}

A set of heuristics are as follows and should help you in assessing the usability of each task. Please read through them and keep them in mind when assessing the system:

Visibility of system status: The system should always keep users informed about what is going on, through appropriate feedback within reasonable time.

Match between system and the real world: The system should speak the users' language, with words, phrases and concepts familiar to the user, rather than system-oriented terms. Follow realworld conventions, making information appear in a natural and logical order.

User control and freedom: Users often choose system functions by mistake and will need a clearly marked "emergency exit" to leave the unwanted state without having to go through an extended dialogue. Support undo and redo.

Consistency and standards: Users should not have to wonder whether different words, situations, or actions mean the same thing. Follow platform conventions.

Error prevention: Even better than good error messages is a careful design which prevents a problem from occurring in the first place. Either eliminate error-prone conditions or check for them and present users with a confirmation option before they commit to the action.

Recognition rather than recall: Minimize the user's memory load by making objects, actions, and options visible. The user should not have to remember information from one part of the dialogue to another. Instructions for use of the system should be visible or easily retrievable whenever appropriate.

Flexibility and efficiency of use: Accelerators -- unseen by the novice user -- may often speed up the interaction for the expert user such that the system can cater to both inexperienced and experienced users. Allow users to tailor frequent actions. 
Aesthetic and minimalist design: Dialogues should not contain information which is irrelevant or rarely needed. Every extra unit of information in a dialogue competes with the relevant units of information and diminishes their relative visibility.

Help users recognize, diagnose, and recover from errors: Error messages should be expressed in plain language (no codes), precisely indicate the problem, and constructively suggest a solution.

Help and documentation: Even though it is better if the system can be used without documentation, it may be necessary to provide help and documentation. Any such information should be easy to search, focused on the user's task, list concrete steps to be carried out, and not be too large.

If you encounter usability problem please describe it in the following manner at the end of this document. The example is filled out with an example problem where the user couldn't tell that the exhibit buttons on the map could be selected because there was no hover effect or indication that they could interact with them until they clicked them.

\section{Usability Problem Example-}

UI element or action triggering problem: Exhibit button on map, when trying to complete the select an exhibit task.

Description of problem: Didn't know you could click on the exhibit buttons on the map because they didn't have a hover effect or any kind of indicator.

Violated Heuristic: Visibility of system status.

Violated Design Principle: I can perform the task but for some users it may take a bit of time to realize you can click those buttons so it makes the system less efficient then it could be.

Severity Rating: I'm going to give it a severity rating of 2. It needs to be fixed as some users may not clue in that you can click those buttons.

0 - I don't agree that this is a usability problem at all.

1 - Cosmetic problem only: need not to be fixed unless extra time is available on project.

2 - Minor usability problem: fixing this should be given a low priority.

3 - Major usability problem: important to fix, so should be given a high priority.

4 - Usability Catastrophe: imperative to fix this before product can be released. 


\section{Appendix G: In-Depth Analysis of Satisfaction Results}

\section{Sub-Test 1:}

Question 11: Which visual narrative helped you reflect upon your experiences the most?

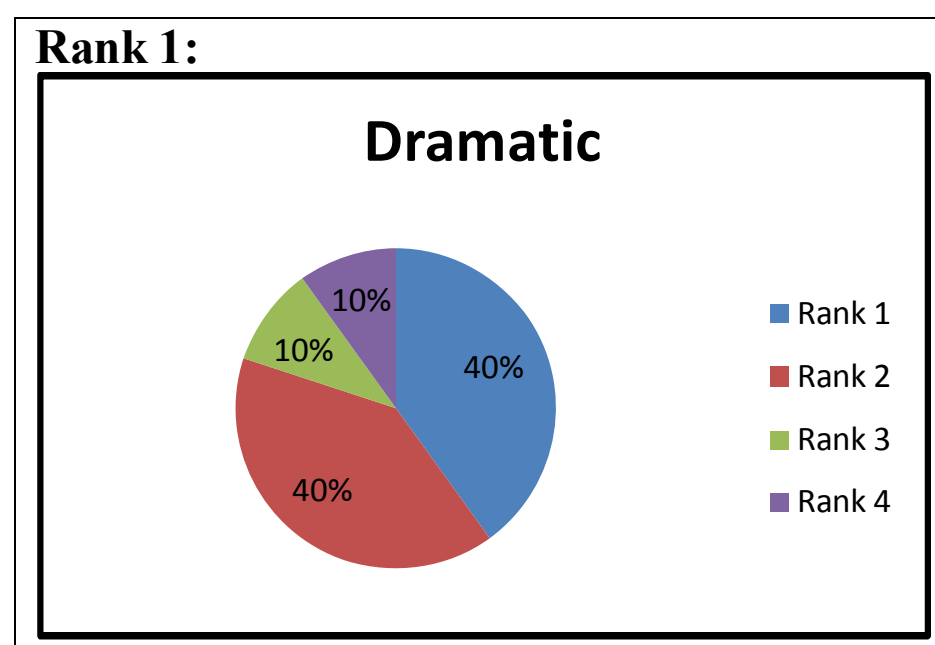

Mode Ranking: 1/2

Average Ranking: 1.9 +/- 0.97 SD.

$80 \%$ of the users gave this visualization a rank of either 1 or 2 with a $40 \%$ split for each rank. In this regard it was ranked the highest overall and was the first or second most favourite in regards to reflection.

\section{Rank 2:}

\section{Sequential}

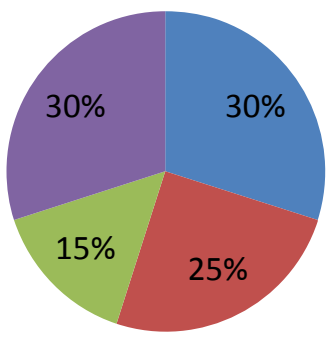

nank 1

- Rank 2

Rank 3

Rank 4

\section{Mode Ranking: 1/4}

Average Ranking: 2.45 +/- 1.23 SD.

With the user rankings for this visualization split almost evenly, how reflective users thought this visualization to be seemed largely due to user preference and perception. However it's average ranking places it as second highest ranked.

\section{Rank 3:}

\section{Rank 4:}

\section{Catagorical}

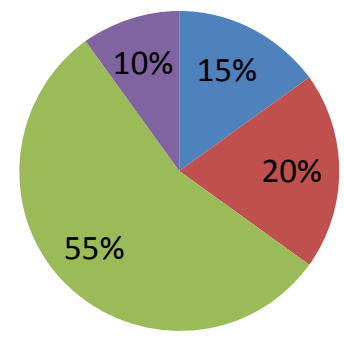

\section{Mode Ranking: 3}

Average Ranking: $2.6+/-0.88$ SD.

With $55 \%$ of the users giving this visualization a rank of 3 this visualization was narrowly the majority of people's third favourite visualization.

\section{Slideshow}

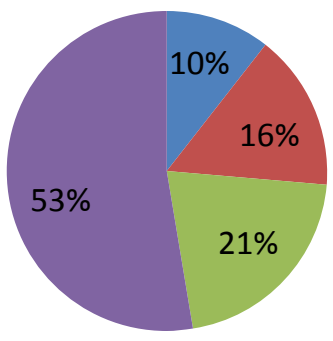

Rank 1

Rank 2

Rank 3

Rank 4

\section{Mode Ranking: 4}

Average Ranking: 3.05 +/- 1.15 SD.

With this visualization 53\% of the participants gave it a rank of 4 , indicating that it was the visualization which was the least helpful for reflection. 
Question 12: Which visual narrative conveyed your experiences the most uniquely?

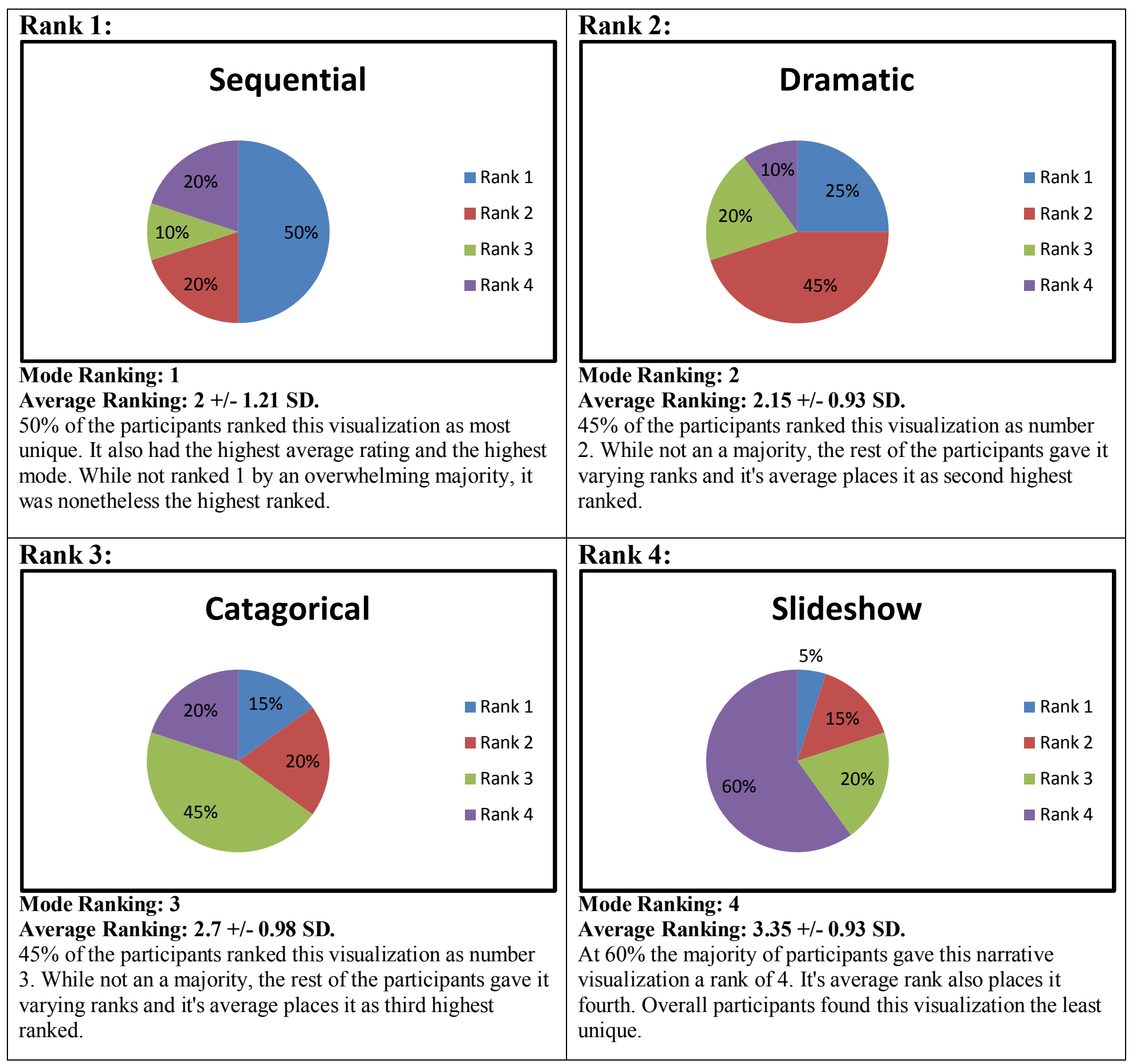


Question 13: Which visual narrative conveyed your experiences most satisfactory?

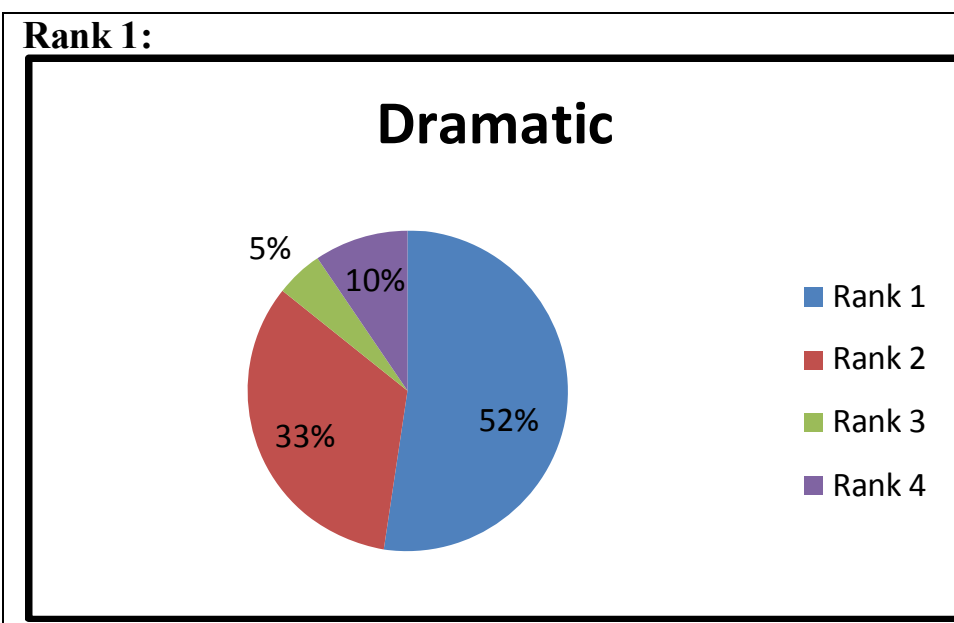

Mode Ranking: 1

Average Ranking: 1.75 +/- 0.97 SD.

$52 \%$ of the participants, a slight majority, ranked this visualization 1 . Additionally $33 \%$ ranked it second, a higher percentage than any other so among those that did not rank it first they ranked it a clear second. However it's average places it as most highest ranked.

\section{Rank 3:}

\section{Sequential}

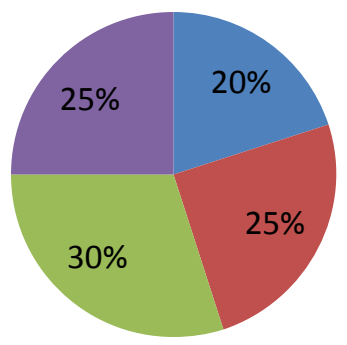

\section{Mode Ranking: 3}

Average Ranking: 2.6 +/- 1.09 SD.

This narrative visualization was ranked very evenly by participants, with 3 rd coming out slightly ahead. It's average ranking also places it as the third highest.
Rank 2:

\section{Catagorical}

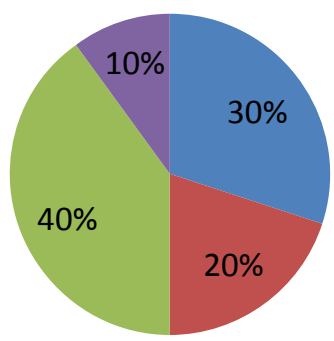

Rank 1

Rank 2

Rank 3

Rank 4

\section{Mode Ranking: 3}

\section{Average Ranking: 2.3 +/- 1.03 SD.}

Because the Dramatic visual narrative was ranked 1st and 2nd by so many participants there isn't really strong trends among the Categorical and the Sequential in regards to which came 3 rd or 4th. However the Categorical one had a slightly higher average rating which places it as the second highest ranked.

\section{Rank 4:}

\section{Slideshow}

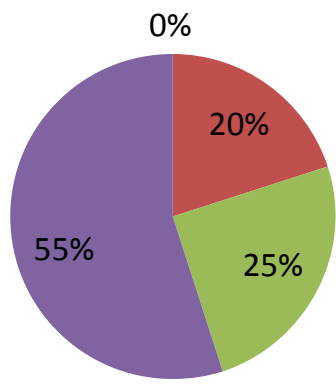

Rank 1

Rank 2

Rank 3

Rank 4

\section{Mode Ranking: 4}

\section{Average Ranking: 3.35 +/- 0.81 SD.}

The majority of users, $55 \%$, ranked this visualization 4 th. As a result this narrative visualization was the least satisfactory and it's average ranking places it lasts. 


\section{Sub-Test 2:}

Question 10: Which was the most engaging?

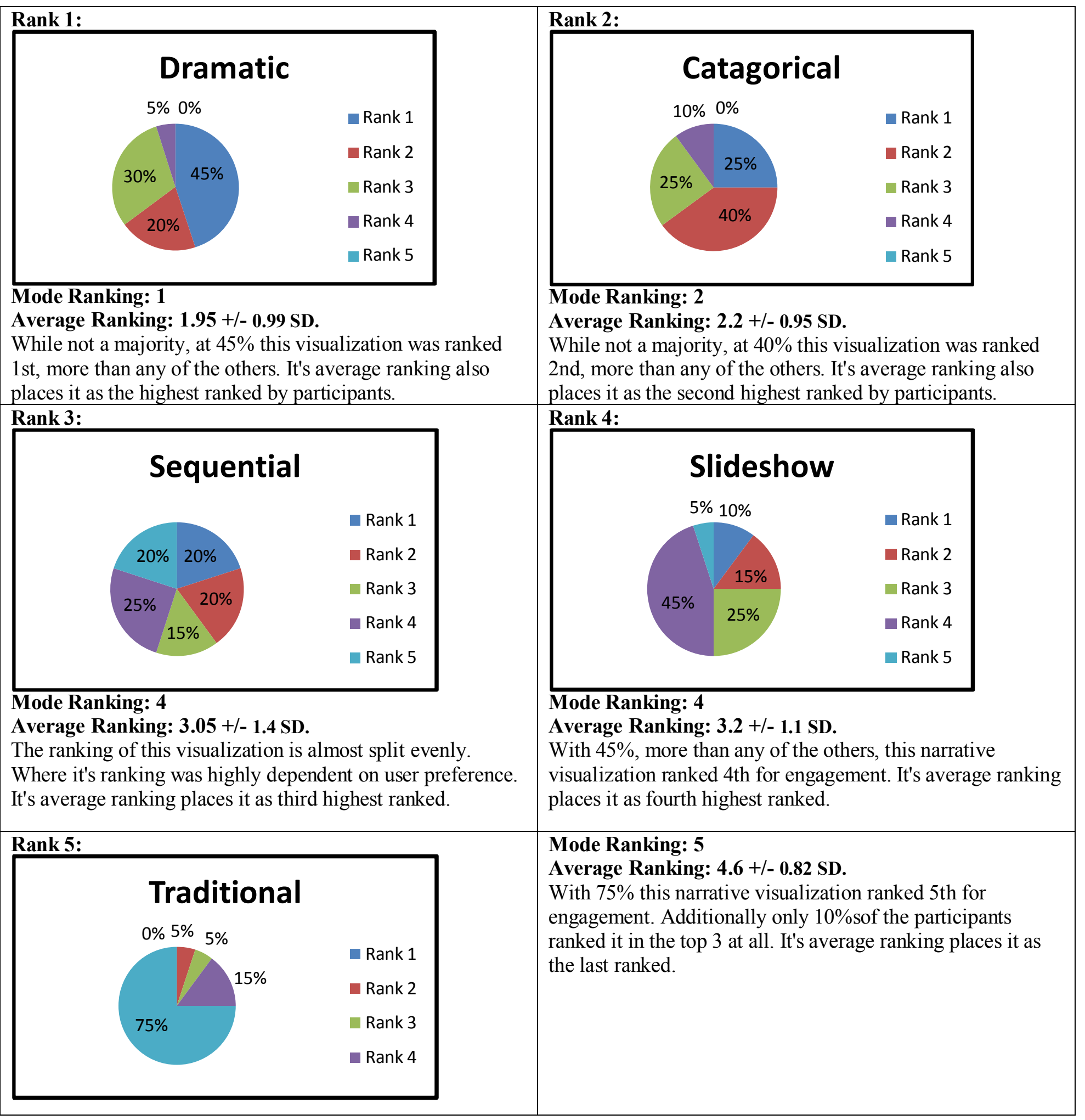


Question 11: Which helped you learn the most about the exhibits?

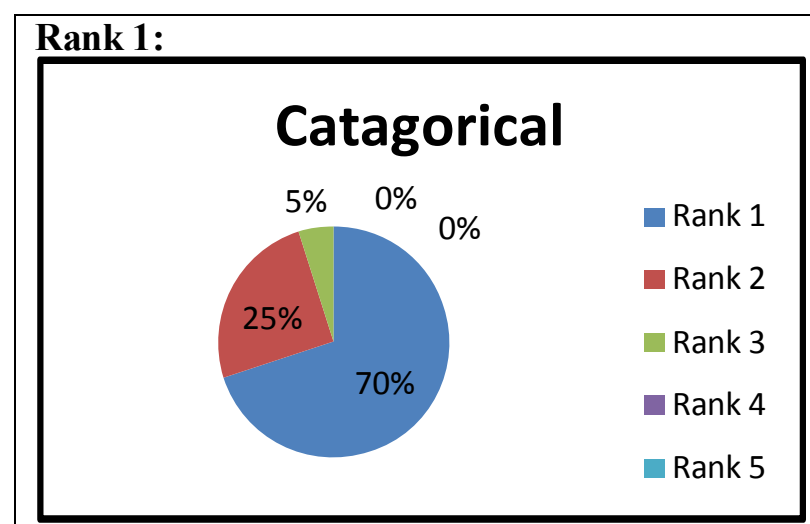

Mode Ranking: 1

Average Ranking: 1.35 +/- 0.58 SD.

With a majority at $70 \%$ this visualization was ranked 1 st by a large margin. It's average ranking places it as the highest ranked by participants.
Rank 2:

\section{Dramatic}

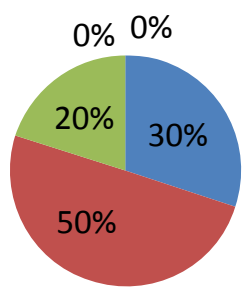

- Rank 1

Rank 2

Rank 3

Rank 4

Rank 5

\section{Mode Ranking: 2}

Average Ranking: 1.9+/- 0.71 SD.

While just under a majority at $50 \%$ this visualization was ranked 2 nd, more than any of the others. It's average ranking also places it as the second highest ranked.

\section{Rank 4:}

\section{Sequential}

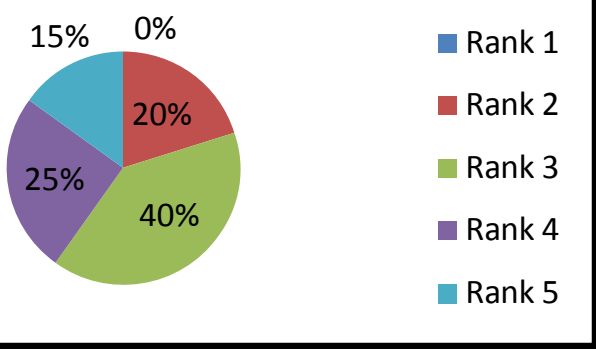

Mode Ranking: 3

Average Ranking: 3.35 +/- 0.99 SD.

While not a majority, at $40 \%$ it ranked 3rd more than any other. It's ranking seems dependent on individual preference, however it's average ranking places it third.

\section{Rank 5:}

\section{Traditional}

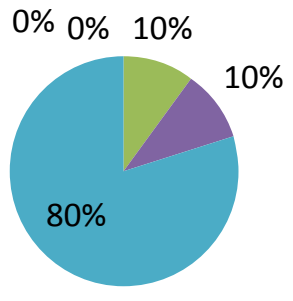

Rank 1

- Rank 2

Rank 3

- Rank 4

- Rank 5
$0 \%$

\section{Slideshow}

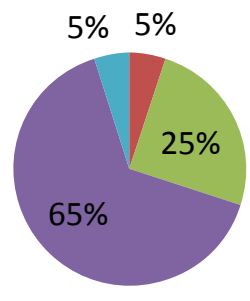

Rank 1

- Rank 2

Rank 3

Rank 4

- Rank 5

\section{Mode Ranking: 4}

Average Ranking: $3.7+/-0.66$ SD.

With $65 \%$, more than any of the others, this narrative visualization ranked 4th for learning. It's average ranking also places it as fourth highest ranked.

\section{Mode Ranking: 5}

Average Ranking: 4.7 +/- 0.66 SD.

With $80 \%$ this narrative visualization ranked 5 th for learning. Additionally only $20 \%$ of the participants ranked it above 5 th at all. It's average ranking places it as the last ranked. 
Question 12: Which communicated the experiences the most clearly?

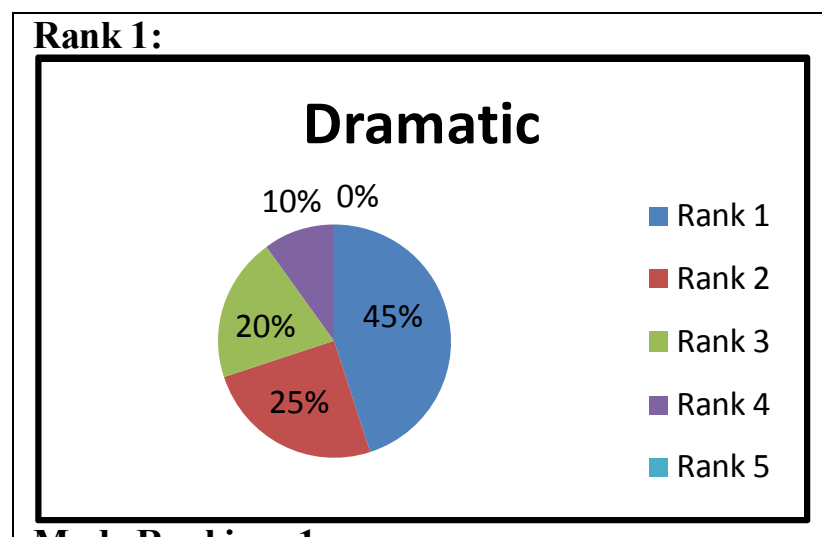

Mode Ranking: 1

Average Ranking: 1.95 +/- 1.05 SD.

While not a majority, at $45 \%$ it ranked 1 st, more than any other. It's ranking seems dependent on individual preference, however it's average ranking places it first.

\section{Rank 3:}

\section{Sequential}

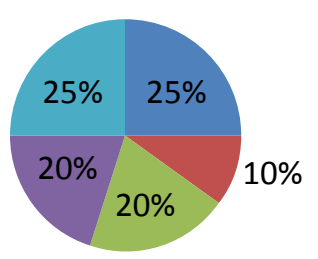

$$
\begin{aligned}
& \text { Rank } 1 \\
& \text { Rank } 2 \\
& \text { Rank } 3 \\
& \text { Rank } 4 \\
& \text { Rank } 5
\end{aligned}
$$

\section{Mode Ranking: 3}

\section{Average Ranking: 3.1 +/- 1.55 SD.}

This narrative visualization had about near equal ranking. It's ranking was highly dependent on participant preference. However it's average places it third highest.

\section{Rank 5:}

\section{Traditional}

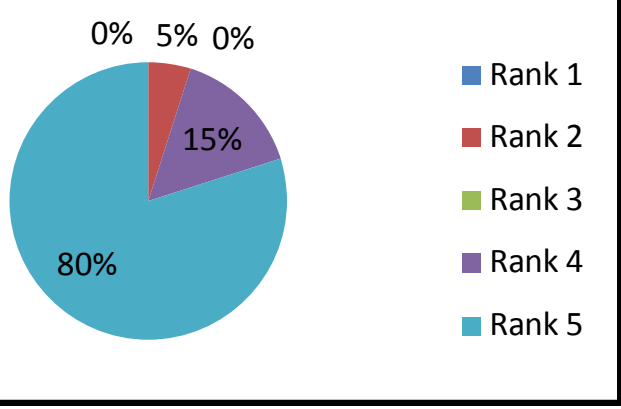

Rank 2:

\section{Catagorical}

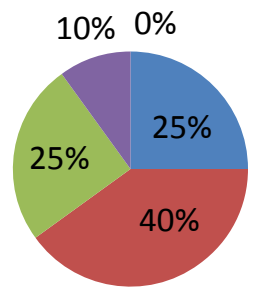

Rank 1

Rank 2

Rank 3

Rank 4

Rank 5

\section{Mode Ranking: 2}

\section{Average Ranking: 2.2 +/- 0.95 SD.}

While not a majority, at $40 \%$ it ranked 2 nd, more than any other. It's ranking seems dependent on individual preference, however it's average ranking places it third.

\section{Rank 4:}

\section{Slideshow}

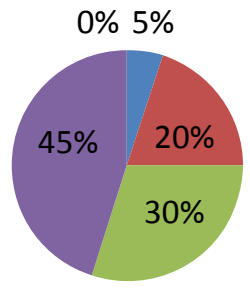

Rank 1

Rank 2

Rank 3

Rank 4

Rank 5

\section{Mode Ranking: 4}

Average Ranking: 3.15 +/- 0.93 SD.

While not a majority, at $45 \%$ it ranked 4 th, more than any other. It's average ranking, although very close to the sequential visualization, places it as fourth highest.

\section{Mode Ranking: 5}

Average Ranking: $4.7+/-0.73$ SD.

$80 \%$ of the users ranked this 5 th. Thus the vast majority found it the one that was the least communicative of museum experiences. Only $20 \%$ of users ranked it above 5 th at all and it's average ranking places it last. 


\section{Appendix H: Sources of Content}

One design consideration was the sources of the content that the narrative visualizations would use to create their visualizations. Where this content came from could be an interesting factor in the design of the system. Four possible sources were discussed:

- The Museum: prepared content from the museum itself that is added into the narrative visualizations based upon what exhibits the user visited.

- The User: content the user has collected in the museum. For example, pictures they took of exhibits.

- Other Users: content that other users have collected during their trip(s). For example pictures they have taken reviews or ratings of exhibits, and other social media type data.

- Outside Sources: sources other than the museum could be used to provide additional information on topics that the exhibits embodied.

Additionally, several museums were visited with the main purpose of classifying the type of exhibits that were common in them. This would help get a better idea of the type of data that could be collected from them and what sources would be most prevalent. The museums visited were the Canadian War Museum [36], The Canadian Museum of Nature [37], and The Canadian Museum of History [38].

While the following list is not exhaustive, it does describe some of the more common exhibit types. They are presented with a classification name, photos of the type of exhibit, and some notes about them. 

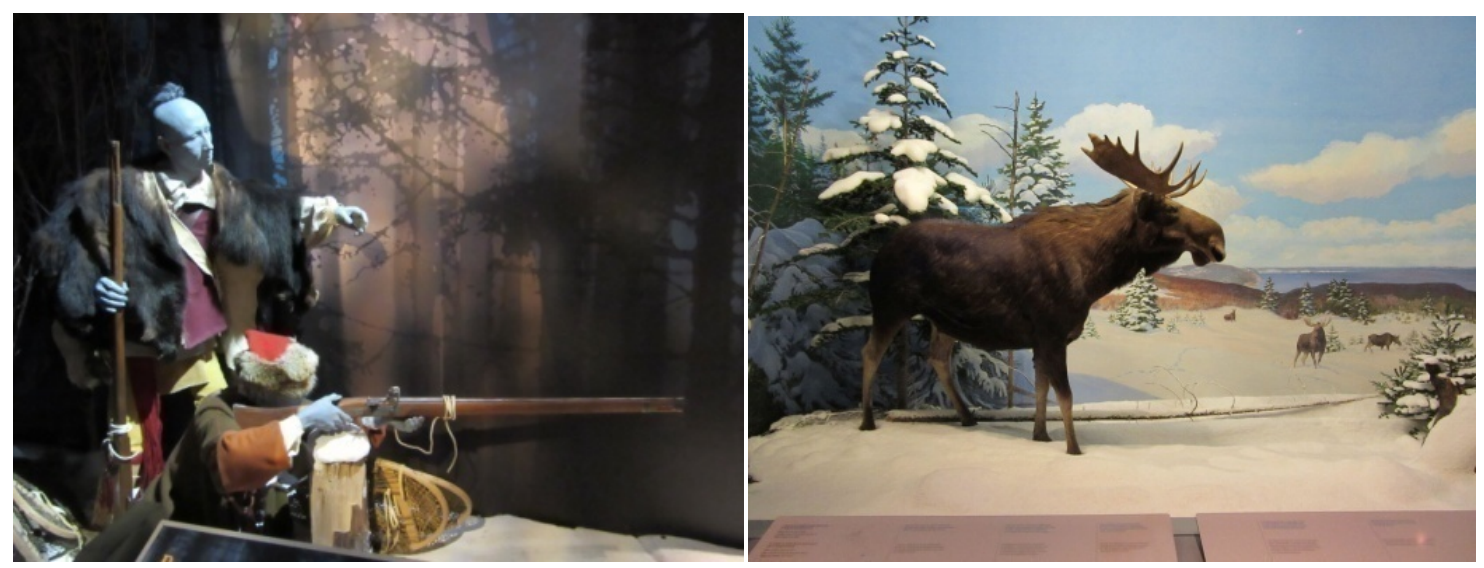

\section{Figure 21. Diorama}

Diorama exhibits are of varying sizes. They can be tiny bird's eye view models of scenes, or life sized representations. What they all have in common is that they are either original or reproductions of artefacts arranged in some kind of scene and try to illustrate some kind of event. Unlike freeform exhibits, dioramas tend to be enclosed and have accompanying information plaques about what is going on in the scene.

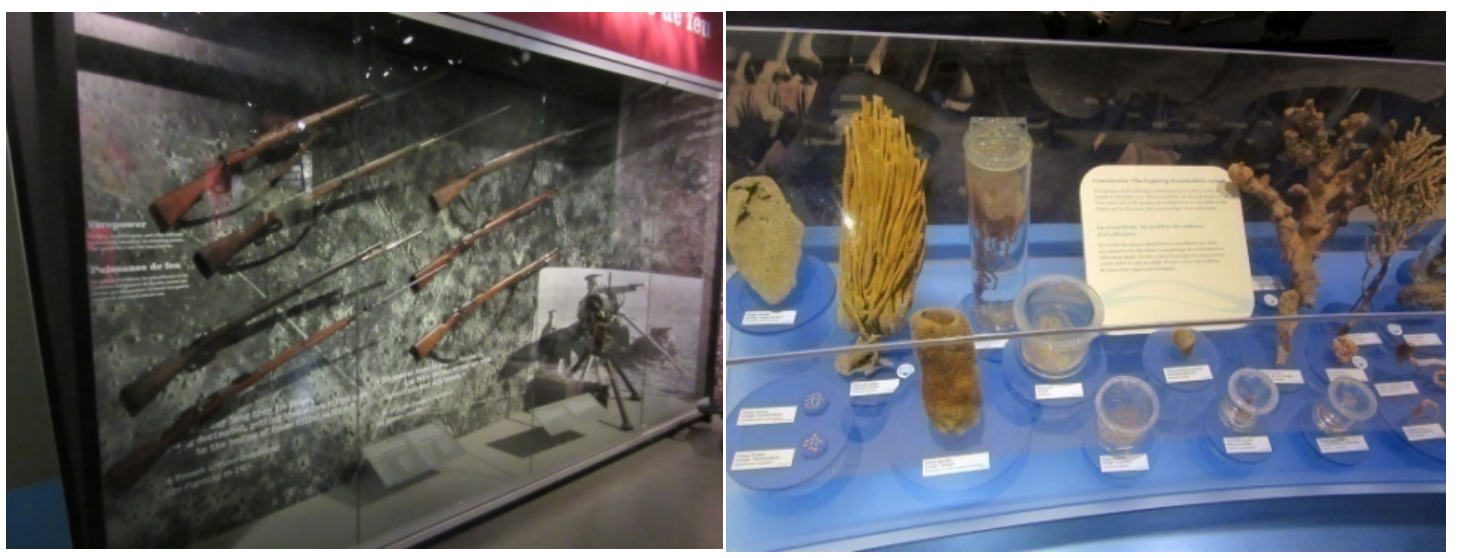

Figure 22. Display Case

Display case exhibits were probably the most prevalent type of exhibit. They consist of a group of artefacts mounted in a display case. The artefacts are named and identified and 
there may be some accompanying information. However, the information is usually about the category the artefacts belong to rather than specific artefacts themselves.

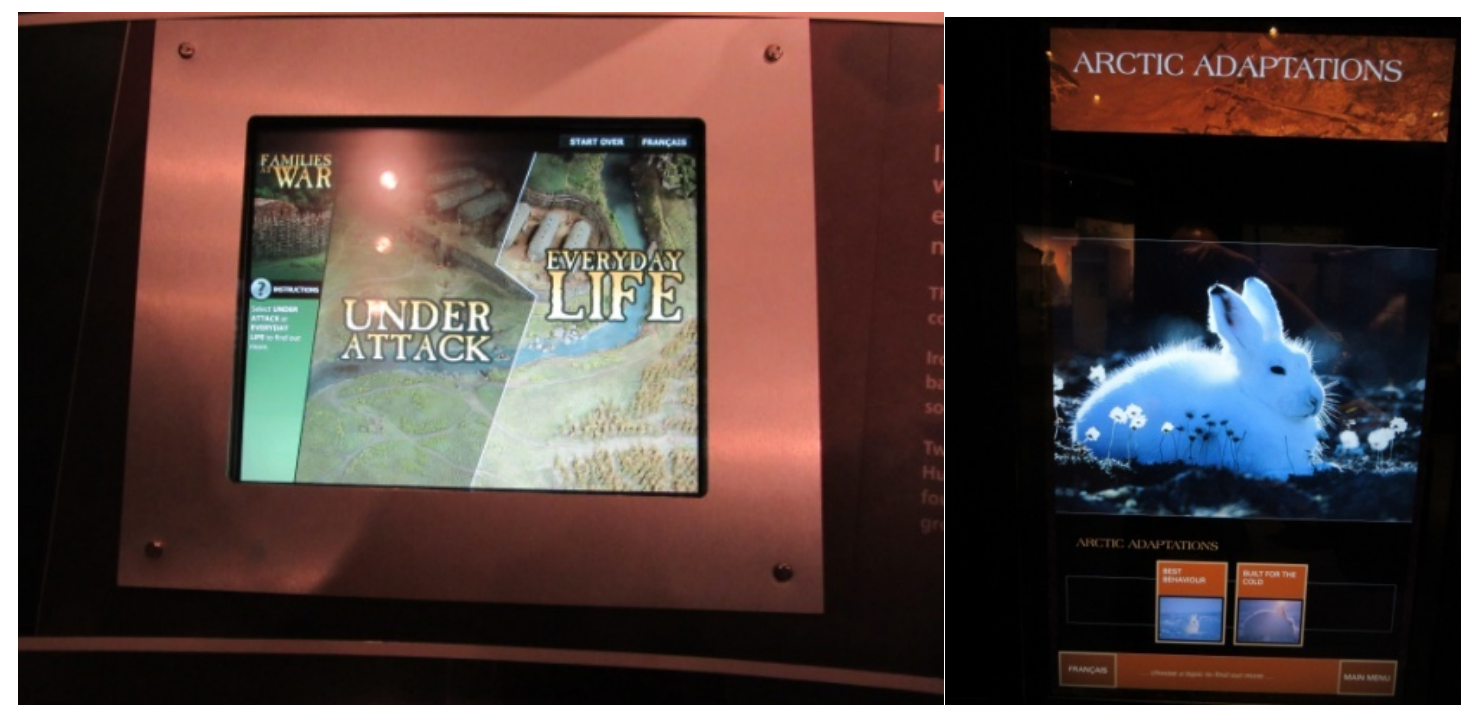

Figure 23. Electronic Quiz

Electronic touch-screen kiosk quizzes were prevalent in several exhibits. They would contain information the user could explore and then be tested on. Overall the interactions are fairly simple in most cases.

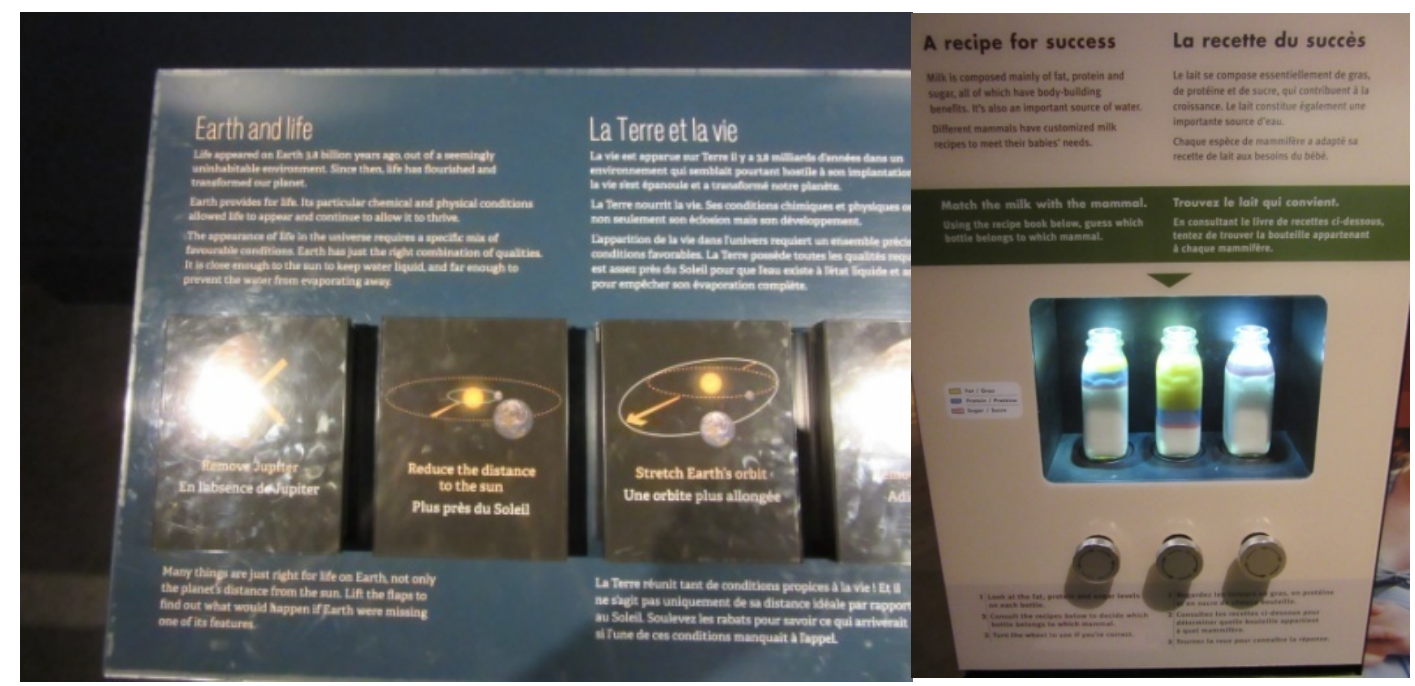

Figure 24. Tactile Quiz 
In contrast to the electronic quizzes, there were also tactile quizzes. These were more common than the electronic ones and would feature some information about a topic and then pose a question. There would be some kind of panel to flip, button to push, or lever to pull, that would reveal the answer. While simple, these seemed to be more popular then the electronic quizzes especially among children. Multiple people can view and participate in the quiz at once and they were often situated at a position that children could reach.
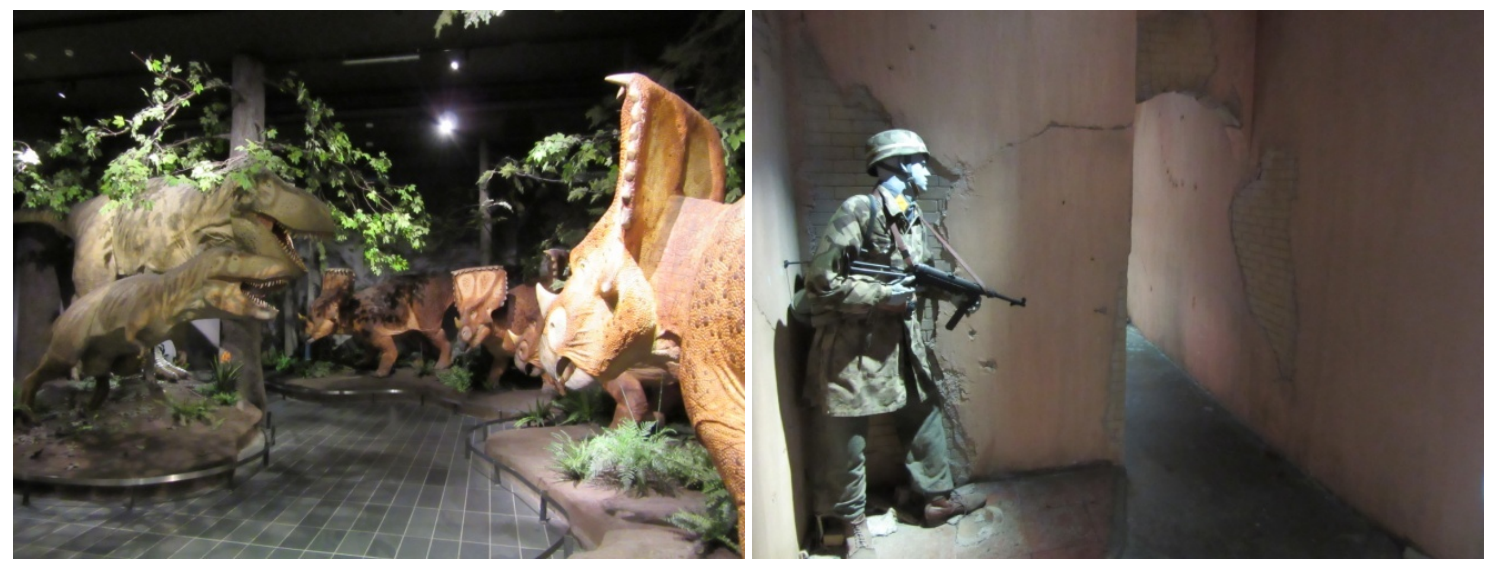

Figure 25. Immersive Tableau

Immersive tableaus were essentially very large dioramas that the person could walk through and immerse themselves in. They were made so that the visitor feels like they are in a scene pertaining to the exhibit. The information about the tableau is generally found in the displays and exhibits preceding the scene. These exhibits seemed to be very popular due to the level of immersion they provide. 


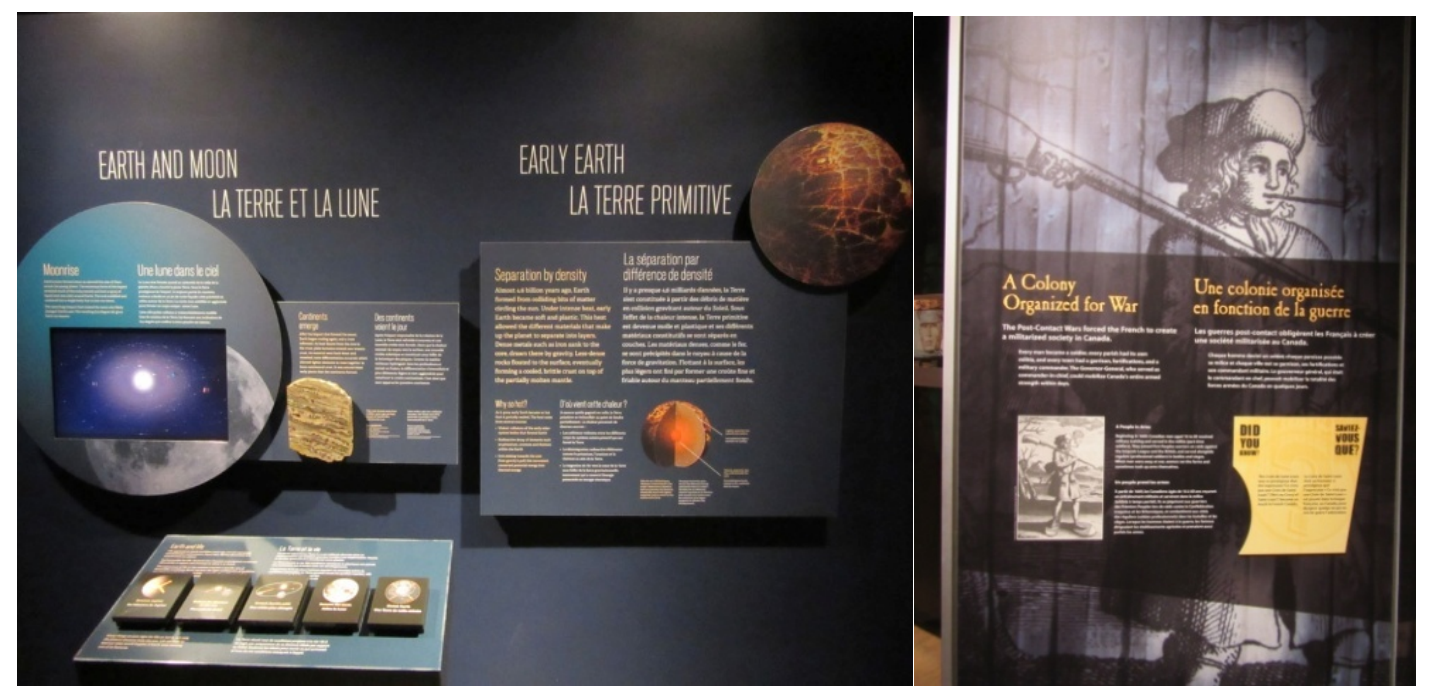

Figure 26. Infowall

Infowalls were a common type of display. There were comprised of text and pictures arranged on a wall. Sometimes with an artefact or two mounted alongside. Overall more common for abstract topics or topics with few physical artefacts.

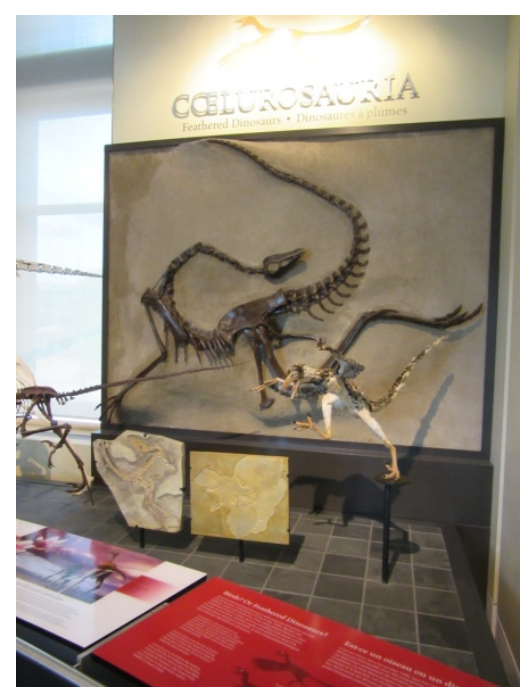

Figure 27. Labelled Display

Labelled displays were another very common type of exhibit, in them an artefact or reproduction is displayed and a panel is mounted at waist level in front of it describing the artefact. While similar to the display case, the labelled display is different in that there 
is only one central artefact and the information tends to be detailed and focused on that artefact.

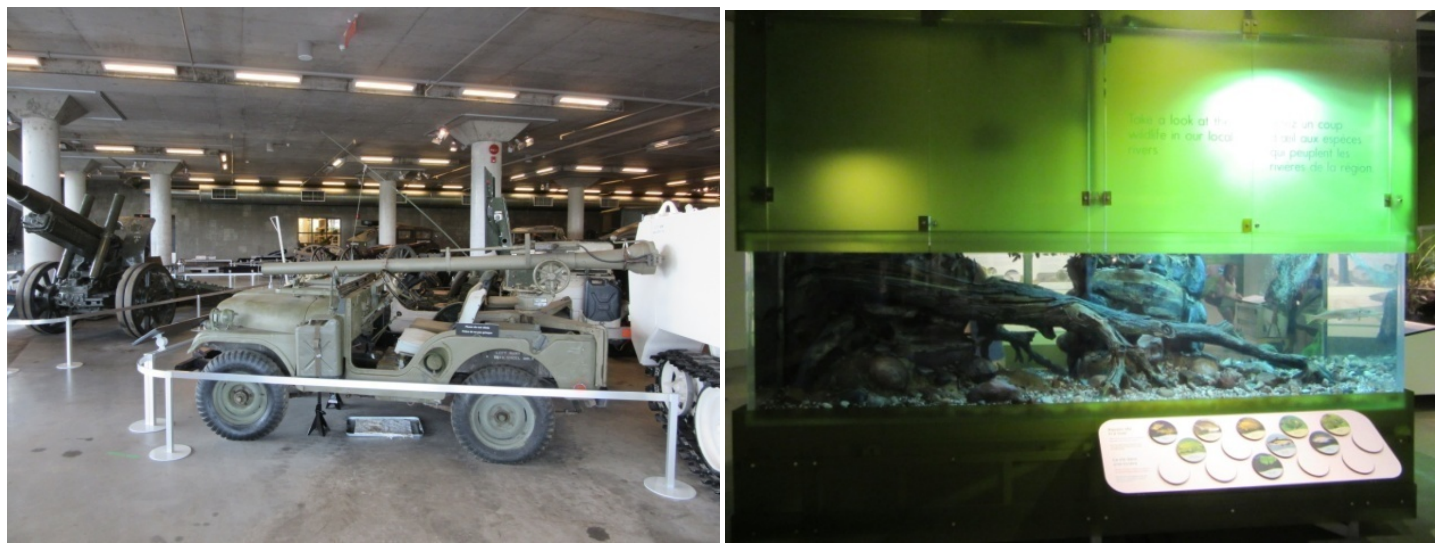

Figure 28. Observable Artefact

Observable artefacts were large artefact presented in a manner that the user could get a really good look at it and be able to view it and all its parts from multiple angles. While there is some information about it, generally it is not as much as other exhibits. Unlike the tableau the user cannot touch the artefact. In the Nature Museum the observable artefacts tended to be live animals like fish or insects. In the War Museum the artefacts tended to be military vehicles and large armaments.

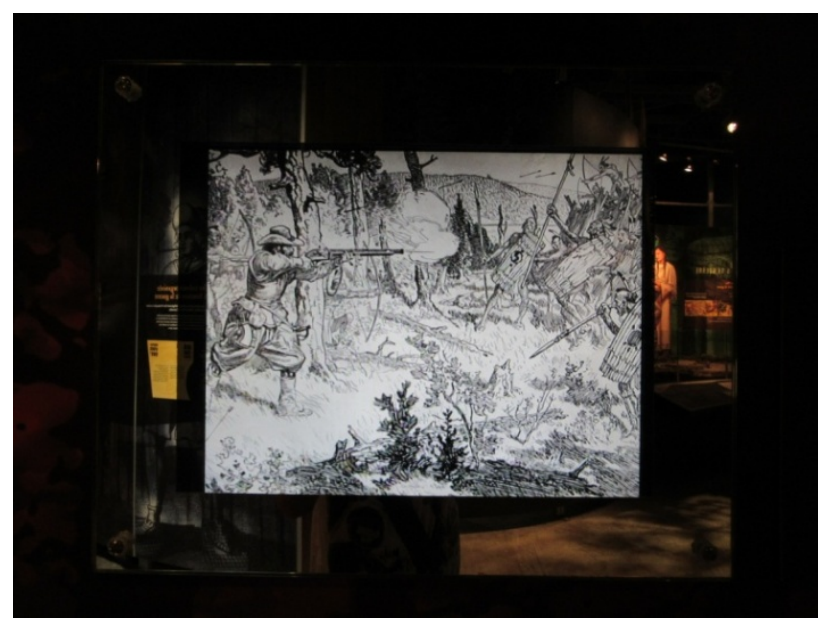

Figure 29. Video Display 
Video exhibits generally displayed information about a topic in the museum. The display could be a small regular TV or a large wall projection. The Museum of Nature didn't really have these displays. They were common at the War Museum to convey historical events which are sequential and can be more easily displayed through video.

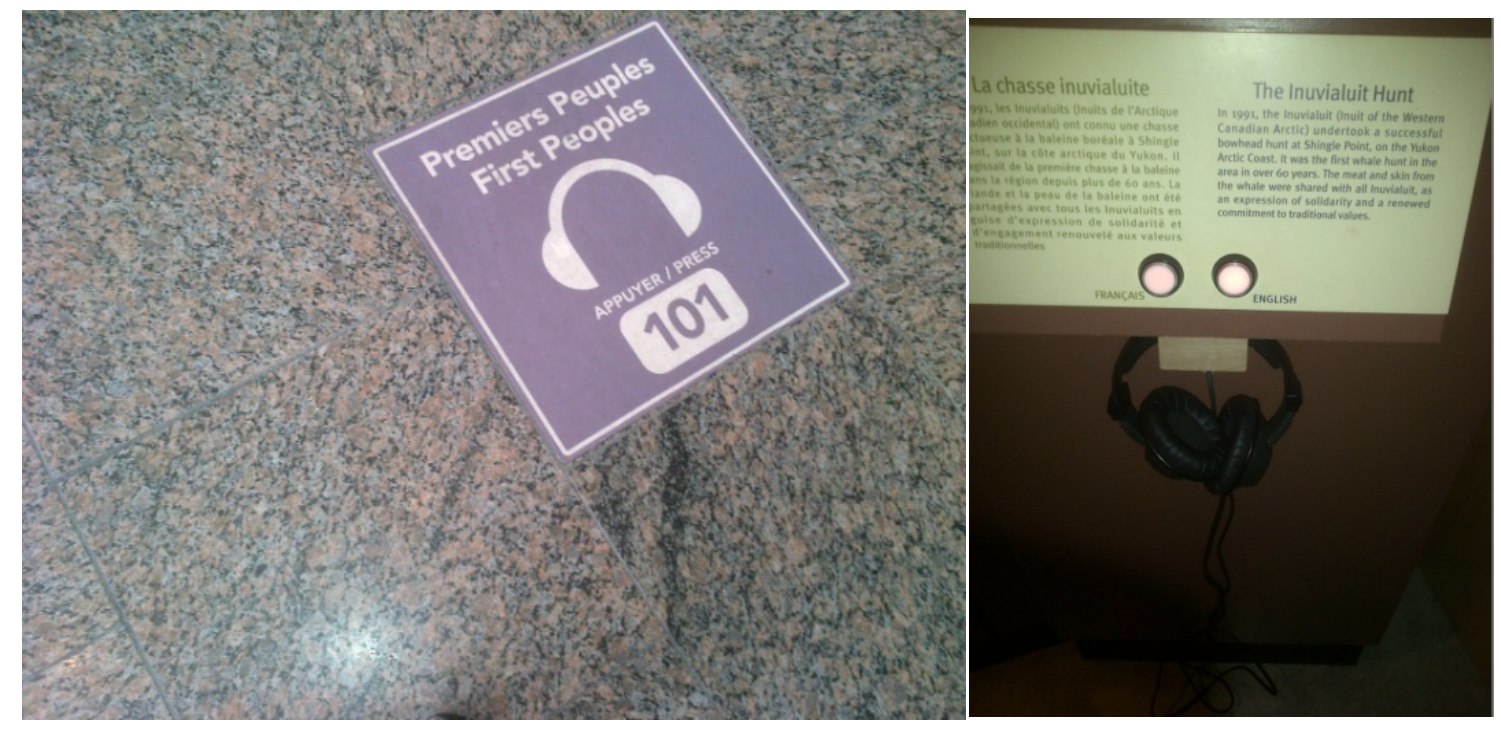

Figure 30. Audio Exhibit

Audio exhibits often accompanied various types of displays. Sometimes the audio would just be playing in the background, narrating the information on the display or adding atmospheric sound effects to the exhibit; sometimes the audio display would consist of a pair of headphones that the visitor could put on and press a button to listen to a story or narration; and sometimes the audio display would consist of a tour where the user could stop at certain points and listen to information about the exhibit. These types of audio displays where present at both Nature and Civilization Museums. 


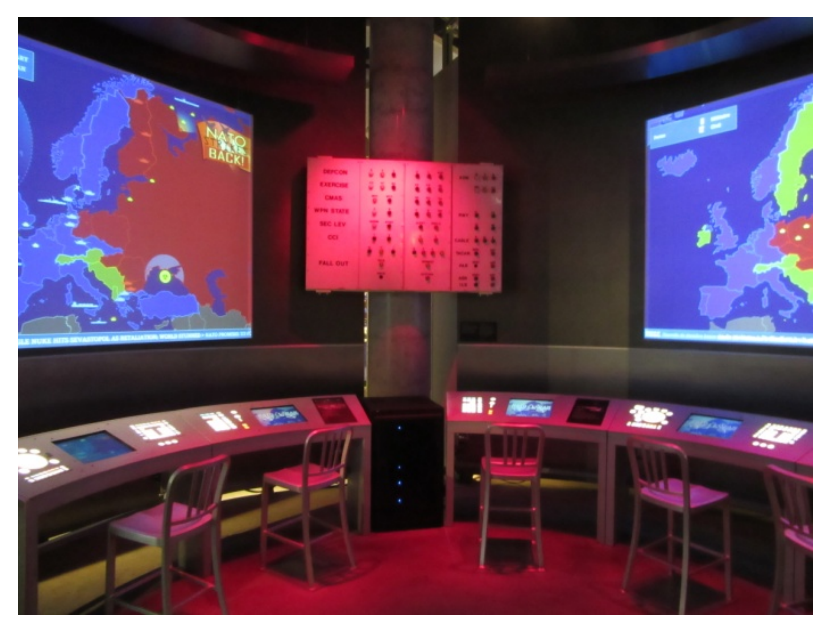

Figure 31. Interactive Game

The interactive game was the most complex type of exhibit and only present at the War Museum. The interactive game exhibit is an exhibit where people can sit down and participate in some kind of complex game against each other. At the War Museum this consisted of a cold war type simulation game where the player would have to try and nuke each other and engage in other cold war military tactics. 